\author{
Universidade de São Paulo \\ Instituto de Astronomia, Geofísica e Ciências Atmosféricas
}

Departamento de Astronomia

Geraldo Gonçalves dos Santos Junior

\title{
Ajustes espectrais de populações estelares: uma análise de confiabilidade
}

São Paulo 

Geraldo Gonçalves dos Santos Junior

\section{Ajustes espectrais de populações estelares: uma análise de confiabilidade}

Dissertação apresentada ao Departamento de Astronomia do Instituto de Astronomia, Geofísica

e Ciências Atmosféricas da Universidade de São Paulo como requisito parcial para a obtenção do título de Mestre em Ciências.

Área de Concentração: Astronomia

Orientadora: Prof ${ }^{\mathrm{a}}$. Dr ${ }^{\mathrm{a}}$.

Paula Rodrigues Teixeira Coelho

Versão corrigida. O original encontra-se disponível na Unidade.

São Paulo 

A todas as mulheres e pessoas $L G B T Q+$ que trabalham no meio científico e que, diariamente, lutam para tornar esse ambiente mais diverso e acolhedor. We'll fight together! 



\section{Agradecimentos}

"There are some things you can't share without ending up liking each other, and knocking out a twelve-foot mountain troll is one of them." - J.K. Rowling, Harry Potter and the Sorcerer's Stone

À minha família, por todo o apoio e preocupação;

À minha orientadora, Professora Paula Coelho, por ter me aceitado como aluno, pela paciência em cada tarde em que sentou do meu lado pra me explicar algo, por ser a melhor professora do mundo, por ter me ensinado boa parte do que eu sei a respeito de Astronomia e Astrofísica, por se preocupar comigo (e não apenas no sentido acadêmico), por tratar a mim e a todos os meus colegas de grupo com o maior respeito e carinho que já recebi de um professor e por ter me guiado durante o desenvolvimento desse projeto;

À Amanda, por me ajudar com matplotlib, por estar sempre presente (mesmo nas vezes em que lhe dei bronca por querer almoçar salgadinho) e por ser a melhor amiga que eu poderia ter;

Aos meus amigos do IAG-USP: Ana Clara, André's (Figueiredo, Rodrigo e Vitorelli), Artur, Bárbara, Catarina, Dai, Dani, Diana, Diego, Erik, Fábio, Fernanda, Geisa, Gustavo, Hélio, Henrique, Ivan, Jhon, Jônatas, Ju, Júlia, Lilianne, Livia, Lory, Luísa, Marcelo, Mirian, Nati, Pablo, Pedro Henrique, Rafa, Roberta, Rubinho, Stela, Stephan, Stephane, Thayse, Ve, Vini, Vitor e Wesley (que é quase do IAG). Obrigado pela ajuda, pelas risadas, pelas festas, pelas inúmeras idas ao bar e por me ouvirem falar (sem parar, eu sei) sobre Taylor Swift. Vocês fizeram essa fase da minha vida infinitamente mais feliz; 
Um agradecimento especial ao Fábio e à Amanda por terem ajudado na revisão deste texto, à Thayse pela ajuda com as referências sobre evolução estelar e evolução química e à Diana por fornecer o material de sua iniciação científica;

Ao Time ${ }^{\circledR}$ : Catarina, Caê, Cris, Fernando, Gabi, Grassetti, Gui, Isa, Mai, Maya, Rafa, Théo, Xii e Yuri. Pela amizade e companhia, por cada rolê, por cada bandejão, por cada madrugada estudando juntos e por terem feito a graduação em Física valer a pena;

Ao Gui, por todo esse tempo de amizade, por me abrigar em Londres e por me levar pra conhecer a cidade; ao Cleyton, pelas idas ao teatro e a shows, e por ser um grande amigo e companheiro em filas e aventuras de fandom; Lívia, Marília e Susane, por todo o tempo que passamos juntos nos últimos quinze anos;

À Mia e ao Black Water Studio, que me ensinaram que sou capaz de muito mais do que eu acreditava ser;

Aos membros do AstroTubers e da \#AstroThreadBR, por me permitirem fazer parte de projetos de divulgação científica tão incríveis;

Aos colegas do Grupo PLEIAD, pela ajuda e por me permitirem ajudar;

Ao Professor Ricardo Schiavon, por me receber durante minha visita ao Astrophysics Research Institute na Liverpool John Moores University, pela ajuda no desenvolvimento deste trabalho e pelos drinks;

To Beatriz Barbuy, Chris Usher, Cid Fernandes, Gustavo Bruzual, Jacopo Chevallard and Stéphane Charlot for the helpful comments about this work;

To Taylor Swift, for speaking my thoughts and feelings through her songs and for being the voice stuck in my head during my best and worst moments. Heartbreak is our national anthem, we sing it proudly! 
"And right there where we stood was holy ground."

- Taylor Swift, Holy Ground

À Universidade de São Paulo;

Ao Instituto de Astronomia, Geofísica e Ciências Atmosféricas;

A todos os funcionários do IAG e do Departamento de Astronomia, que nos forneceram café, limparam nossas salas, resolveram nossas burocracias e consertaram nossos computadores.

"I know. It's all wrong. By rights we shouldn't even be here. But we are. It's like in the great stories, Mr. Frodo. The ones that really mattered. Full of darkness and danger, they were. And sometimes you didn't want to know the end. Because how could the end be happy? How could the world go back to the way it was when so much bad had happened? But in the end, it's only a passing thing, this shadow. Even darkness must pass." - Samwise Gamgee, The Lord of the Rings: The Two Towers, J.R.R. Tolkien

À CAPES pelo financiamento desse trabalho, apesar dos cortes de verba e do plano de desmonte da Educação e da Ciência brasileiras posto em prática pelo atual governo do país;

A todos os professores, alunos, funcionários e políticos que, em meio a essa onda de terror e obscurantismo, tem lutado a favor da Ciência e de uma Universidade pública e inclusiva;

A todos os cidadãos brasileiros que votaram contra o atual presidente.

Esta tese/dissertação foi escrita em LATEX com a classe IAGTESE, para teses e dissertações do IAG. 

"Doubt was much more energy efficient than conviction." - Gregory Maguire, Wicked: The Life and Times of the Wicked Witch of the West

"A philosopher once asked,

'Are we human because we gaze at the stars,

or do we gaze at them because we are human?'

Pointless, really...

'Do the stars gaze back?'

Now, THAT's a question."

- Neil Gaiman, Stardust

"Don't you see the starlight? Don't you dream impossible things?"

- Taylor Swift, Starlight 



\section{Resumo}

O ajuste espectral pixel-a-pixel é uma técnica amplamente utilizada nos estudos de populações estelares. Ela permite ao usuário inferir diferentes parâmetros (como idades e abundâncias químicas) a partir de espectros de luz integrada de galáxias e aglomerados de estrelas. Nesse trabalho, estudamos populações estelares de aglomerados globulares e examinamos o quanto a escolha do intervalo de comprimento de onda afeta os parâmetros ajustados. Utilizamos duas bibliotecas de espectros de luz integrada de aglomerados globulares e os ajustamos com modelos de população estelar simples utilizando o código Starlight. Fizemos testes usando diferentes regiões espectrais de modo a inferir valores de avermelhamento, idades, $[\mathrm{Fe} / \mathrm{H}]$ e $[\alpha / \mathrm{Fe}]$. Comparando nossos valores com idades obtidas a partir de ajustes de isócronas e abundâncias químicas de espectroscopia de alta resolução, concluímos que: (1) os resultados obtidos variam dependendo do intervalo de comprimento de onda usado; (2) o método, em geral, retorna boas estimativas de avermelhamento, especialmente quando intervalos maiores são utilizados nos ajustes; (3) a região espectral mais adequada para determinação de idades, $[\mathrm{Fe} / \mathrm{H}]$ e $[\alpha / \mathrm{Fe}]$ dentre os interva-

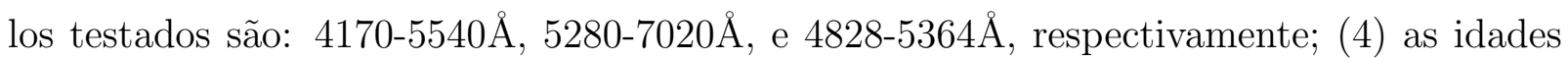
obtidas para objetos pobres em metais podem ser vários bilhões de anos mais jovens que as idades de isócronas. Concluímos que, dependendo dos parâmetros de interesse e da acurácia desejada, ajustar o maior intervalo possível de comprimento de onda pode não ser a melhor estratégia. Com as informações obtidas do estudo com aglomerados globulares, analisamos a luz integrada de uma amostra de galáxias próximas. Nossos resultados concordam com a literatura, galáxias elípticas sendo mais velhas e mais ricas em metais que galáxias disco. Analogamente, populações de bojos clássicos tendem a ser mais velhos e mais ricos em metais do que pseudo-bojos. 



\section{Abstract}

The pixel-to-pixel spectral fitting technique is often used in studies of stellar populations. It enables the user to infer several parameters from integrated light spectra such as ages and chemical abundances of galaxies and star clusters. In this work, we study stellar populations of globular clusters (GCs) and examine the question of how the inferred parameters change with the choice of wavelength range used. We have employed two different libraries of integrated light spectra of GCs from the literature and fitted them to stellar population models using the code STARLiGHT. We performed tests using different regions of the spectra to infer reddening, ages, $[\mathrm{Fe} / \mathrm{H}]$ and $[\alpha / \mathrm{Fe}]$. Comparing our values to age values obtained from isochrone fitting and chemical abundances from high resolution spectroscopy, we find that: (1) the inferred parameters change with the wavelength range used; (2) the method in general retrieves good reddening estimates, specially when a wider wavelength range is fitted; (3) the most appropriate spectral regions for determination of age, $[\mathrm{Fe} / \mathrm{H}]$, and $[\alpha / \mathrm{Fe}]$ among the ones we tested are: $4170-5540 \AA, 5280-7020 \AA$, and 4828 $5364 \AA$, respectively; (4) the retrieved age values for old metal-poor objects can be several Gyr younger than those obtained from isochrone fitting. We conclude that, depending on the parameter of interest and the accuracy requirements, fitting the largest possible wavelength range may not be the best strategy. With the information obtained through studying GCs, we analysed integrated light from a sample of nearby galaxies. Our results are in agreement with literature, with elliptical galaxies being older and more metal-rich than the stellar populations of disk galaxies. Similarly, populations in classical bulges of spiral galaxies tend to be older and more metal-rich than pseudo-bulges. 



\section{Lista de Figuras}

1.1 Representação visual (e simplificada) do surgimento das Populações I, II e III na história do Universo. Figura de Till $(2017)$. . . . . . . . . . . . . . 26

1.2 Figura 1 de McWilliam (1997). Esquema do comportamento das abundâncias de elementos- $\alpha$ com a metalicidade para a vizinhança solar. . . . . . . . . .

1.3 Aglomerado Globular NGC 104 (47Tuc). Composição de imagens das bandas U, R e uma banda estreita centrada em $485 \mathrm{~nm}$, observadas com o FORS1, no VLT. Créditos da imagem: ESO Science Archive, Rubina Kotak (ESO) e Henri Boffin (ESO). . . . . . . . . . . . . . . .

1.4 Distribuição dos aglomerados globulares observados da Via Láctea. Figura

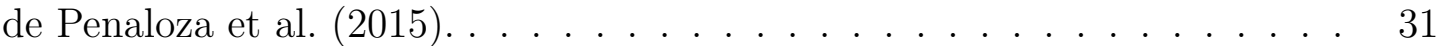

2.1 Distribuições de idade e abundâncias químicas dos aglomerados globulares presentes na biblioteca WAGGS. . . . . . . . . . . . . . . . 36

2.2 Espectros dos aglomerados NGC1851, NGC6333 e NGC6522 das bibliotecas WAGGS (azul) e Schiavon et al. 2005 (verde) sobrepostos. . . . . . . . . . 39

2.3 Distribuição de valores de sinal-ruído (SNRs) dos espectros da biblioteca WAGGS

2.4 Distribuição de valores de sinal-ruído (SNRs) dos espectros de bojos de galáxias utilizados neste trabalho. . . . . . . . . . . 45

2.5 Exemplos de galáxias da amostra de Gadotti (2009). . . . . . . . . . . . 46

2.6 Distribuição de morfologias das galáxias da amostra de Gadotti (2009). . 48

3.1 Modelos de população estelar simples da biblioteca de Vazdekis et al. (2015). 50 
3.2 Representação visual da equação 3.1. A primeira imagem representa uma galáxia observada, enquanto as demais representam populações estelares simples. Imagens: NASA, ESA, Hubble. . . . . . . . . . . . . 51

3.3 Exemplo de ajuste espectral feito pelo Starlight. . . . . . . . . . 52

3.4 Resolução por comprimento de onda para os dados da biblioteca WAGGS (U7000, B7000, R7000 e I7000, respectivamente). Crédito: Christopher Usher (comunicação privada). . . . . . . . . . . . . . . . . .

4.1 Distribuições de $\chi^{2}$ (primeira linha) e $\Delta_{\lambda}$ (segunda) para os ajustes feitos com Starlight dos espectros da biblioteca WAGGS. . . . . . . . . . . 58

4.2 Valores de $A_{V}$ calculados pelo Starlight comparados com valores de referência. ......................... 60 60.

4.3 Idades resultantes dos ajustes espectrais (eixo y) versus idades provenientes de ajustes de isócrona (eixo $\mathrm{x}$ ), em escala logarítimica. . . . . . . . . 62

4.4 Idades resultantes dos ajustes espectrais (eixo y) versus idades provenientes de ajustes de isócrona (eixo $\mathrm{x}$ ), em escala linear. . . . . . . . . . 63

4.5 Valores de $\Delta t=t_{\text {Starlight }}-t_{\text {iso }}$ (ponderados por luz no painel superior e por massa no painel inferior) versus metalicidade, resultantes do ajuste utilizando o intervalo $R_{W}$. . . . . . . . . . . . . . . .

4.6 Espectro de NGC6397 e o modelo de SSP de Vazdekis et al. (2015) que mais se aproxima dos valores de referência para esse aglomerado. . . . . . . . 64

4.7 Representação visual do resultado do melhor ajuste em $R_{B}$ para o espectro de NGC6397 da biblioteca WAGGS. . . . . . . . . . . . . . 66

4.8 Representação visual do resultado do melhor ajuste em $R_{B}$ para o espectro de NGC1916 da biblioteca WAGGS. . . . . . . . . . . . . . . 66

4.9 Valores de metalicidade resultantes dos ajustes espectrais (eixo y) versus valores de referencia $($ eixo $\mathrm{x}) . \ldots \ldots \ldots$

4.10 Distribuições de erros nos cálculos de abundâncias de elementos- $\alpha$. . . . . 69 
4.11 Valores de idade e metalicidade ponderados por massa resultantes dos ajustes feitos com o Starlight dos espectros da biblioteca WAGGS versus os resultados do mesmo processo, porém utilizando os espectros da biblioteca de Schiavon et al. (2005), e distribuição de erros de abundâncias de elementos- $\alpha \ldots \ldots \ldots \ldots \ldots \ldots \ldots$

5.1 Distribuições de $A_{V}$ calculados pelo StarLight para os espectros das galáxias da amostra de Gadotti (2009) observados pelo SDSS. . . . . . . . . . . . 74

5.2 Parâmetroos de população estelar ajustados pelo STARLiGHT para os espectros das galáxias da amostra de Gadotti (2009) observados pelo SDSS. . . .

5.3 Valores de $A_{V}$ ajustados pelo Starlight para os espectros das galáxias da amostra de Gadotti (2009) observados pelo SDSS. . . . . . . . . . . . . . . 76

5.4 Valores de metalicidade ponderados por luz ajustados pelo StARLight para os espectros das galáxias da amostra de Gadotti (2009) observados pelo SDSS. 76

5.5 Valores de idade ponderados por luz ajustados pelo Starlight para os espectros das galáxias da amostra de Gadotti (2009) observados pelo SDSS. 77

5.6 Matriz de correlações para todos os parâmetros (morfológicos e de população estelar) para galáxias AGN com pseudo bojo sem barra da amostra de Ga$\operatorname{dotti}(2009) \ldots \ldots \ldots \ldots \ldots$. . . . . . . . . . . . . . . . . . . . . . . .

D.1 Análogo à Figura 5.6, porém para galáxias de bojo clássico com AGN. . . . 122

D.2 Análogo à Figura 5.6, porém para galáxias de bojo clássico sem AGN. . . . 123

D.3 Análogo à Figura 5.6, porém para galáxias de bojo clássico com barra e com AGN.

D.4 Análogo à Figura 5.6, porém para galáxias de bojo clássico com barra e sem AGN.

D.5 Análogo à Figura 5.6, porém para galáxias de bojo clássico sem barra e com AGN.

D.6 Análogo à Figura 5.6, porém para galáxias de bojo clássico sem barra e sem AGN.

D.7 Análogo à Figura 5.6, porém para galáxias de pseudo-bojo com AGN. . . . 128

D.8 Análogo à Figura 5.6, porém para galáxias de pseudo-bojo sem AGN. . . . 129 
D.9 Análogo à Figura 5.6, porém para galáxias de pseudo-bojo com barra e com AGN. . . . . . . . . . . . . . . . . . . . . . 130

D.10 Análogo à Figura 5.6, porém para galáxias de pseudo-bojo com barra e sem AGN . . . . . . . . . . . . . . . . . . . 131

D.11 Análogo à Figura 5.6, porém para galáxias de pseudo-bojo sem barra e sem AGN. . . . . . . . . . . . . . . . . . . . . . . . 132

D.12 Análogo à Figura 5.6, porém para galáxias elípticas com AGN. . . . . . . . 133

D.13 Análogo à Figura 5.6, porém para galáxias elípticas sem AGN. . . . . . . . 134

D.14 Análogo à Figura 5.6, porém para galáxias espirais com barra com AGN. . 135

D.15 Análogo à Figura 5.6, porém para galáxias espirais com barra sem AGN. . 136

D.16 Análogo à Figura 5.6, porém para galáxias espirais sem barra e com AGN. 137

D.17 Análogo à Figura 5.6, porém para galáxias espirais sem barra e sem AGN. 138 


\section{Lista de Tabelas}

2.1 Intervalos de comprimento de onda dos espectros da biblioteca WAGGS . .

2.2 Aglomerados globulares presentes na base de dados da biblioteca WAGGS e valores de referência para idades e abundâncias químicas. O símbolo '*, indica valores não encontrados em nossa busca. . . . . . . . . . . . . . . 40

2.3 Parâmetros morfológicos e estruturais disponíveis para as 946 galáxias do conjunto de Gadotti $(2009) \ldots \ldots \ldots \ldots$. . . . . . . . . . . . . . . . . . . . .

4.1 Intervalos de cobertura espectral utilizados neste trabalho. . . . . . . . . 5 58

4.2 Desvio padrão típico dos parâmetros obtidos do ajuste de espéctros perturbados utilizando seus respectivos espectros de erro. . . . . . . . . . . . 70

4.3 Valores medianos de $\Delta$ parâmetros \pm intervalos interquartis (IQR) para cada intervalo de comprimento de onda. $\Delta P=P_{\text {Starlight }}-P_{\text {Reference }}$ onde $P$ representa cada parâmetro. Colunas $2-6$ indicam os intervalos ajustados, como definidos na Tabela 4.1 . . . . . . . . . . . . . . . . . . . .

5.1 Pares de parâmetros (morfologia/população estelar) que mostram correlações moderadas.

A.1 Velocidades radiais (calculadas como descrito na seção 3.3) e razão sinalruído médio para cada espectro da biblioteca WAGGS.

C.1 Resultados do ajuste em $R_{W}$ e incertezas provenientes do ajuste de espectros perturbados da biblioteca WAGGS. . . . . . . . . . . . 



\section{Sumário}

1. Introdução . . . . . . . . . . . . . . . . . . . . . . 23

1.1 Populações Estelares . . . . . . . . . . . . . . . . . . . . . . 24

1.2 Aglomerados Globulares . . . . . . . . . . . . . . . . . . . . . . 28

1.3 Ajuste Espectral . . . . . . . . . . . . . . . . . . . . . . . . . 29

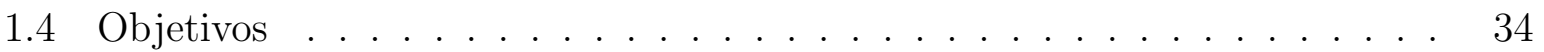

2. Bases de dados . . . . . . . . . . . . . . . . . . . . . . . . 35

2.1 Bibliotecas de luz integrada de aglomerados globulares . . . . . . . . 35

2.2 Uma amostra de bojos galácticos do Universo próximo . . . . . . . . . . 444

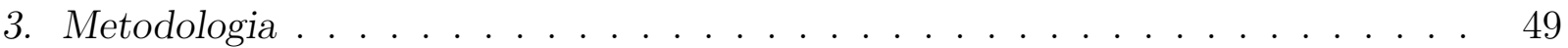

3.1 Modelos de população estelar simples . . . . . . . . . . . . . . . 49

3.2 Starlight: Software de ajuste espectral . . . . . . . . . . . . . . 49

3.3 Pré-processamento dos dados da biblioteca WAGGS . . . . . . . . . . . 53

4. Resultados e análises . . . . . . . . . . . . . . . . 57

4.1 Qualidade dos ajustes ..................... 57

4.2 Avermelhamento . . . . . . . . . . . . . . . . . 59

4.3 Idades . . . . . . . . . . . . . . . . . . . . . . . 60 60

4.4 Abundâncias químicas . . . . . . . . . . . . . . . . . 65

4.5 Incertezas . . . . . . . . . . . . . . . . . . . 67

4.5.1 Incertezas devido ao SNR dos espectros . . . . . . . . . . . . 69

4.5.2 Incertezas vindas de diferentes observações . . . . . . . . . . . . . . 70

4.6 Sumário de resultados . . . . . . . . . . . . . . . . . . . . . . . 70 
5. Aplicação: bojos de galáxias do SDSS . . . . . . . . . . . . . . . 77

5.1 Resultados do ajuste espectral . . . . . . . . . . . . . . 7 73

5.2 Comparação com a literatura . . . . . . . . . . . . . . . 74

5.3 Correlações com morfologia . . . . . . . . . . . . . . . . . . 77

6. Conclusões. . . . . . . . . . . . . . . . . . . . . . . . . . . . 81

Referências ........................ 85

Apêndice 101

A. Velocidades radiais e valores de sinal-ruído . . . . . . . . . . . . 103

B. Script usado na convolução dos espectros . . . . . . . . . . . . . . . 113

C. Resultados do ajuste em $R_{W} \ldots \ldots \ldots \ldots \ldots \ldots$. . . . . . . . . . . .

D. Matrizes de correlação . . . . . . . . . . . . . . . . . . . . . . 121 
Capítulo 1

\section{Introdução}

"We are made of starstuff."

A frase dita por Carl Sagan em seu livro Cosmos (Sagan, 1980) sumariza uma das maiores descobertas astronômicas da História: a existência dos planetas, da Vida e de tudo o que observamos ao nosso redor se deve às estrelas. Elas são as principais responsáveis pela grande diversidade de elementos químicos existentes no Universo, que formam desde galáxias a milhões de parsec de distância até as moléculas do nosso DNA.

Nós, enquanto seres humanos, temos olhado para o céu e tentado entender a origem desses pequenos pontos brilhantes desde os primórdios da nossa espécie. O registro mais antigo da tentativa de estudo das estrelas data do final do segundo milênio a.C. na Babilônia, quando os primeiros catálogos foram compilados. Desde então, astrônomos tem observado o céu noturno em busca de respostas, e seus registros nos permitem mapear a construção do nosso conhecimento sobre o céu: a primeira observação de uma supernova ocorre em 185 d.C. por astrônomos chineses. Astrônomos islâmicos, por volta do ano 1000, constroem os primeiros observatórios e começam a medir a posição das estrelas, além de serem responsáveis pelo primeiro registro da descoberta de um aglomerado globular. Os primeiros astrônomos europeus, como Tycho Brahe, sugeriram que a distribuição das estrelas que víamos no céu não era imutável. A ideia de que as estrelas eram astros como o Sol veio de Giordano Bruno (1584), e Isaac Newton (1687) imaginou que elas poderiam estar espalhadas uniformemente em todas as direções (Kennedy 1962, Jones et al. 1991, Zhao et al. 2006).

Uma grande revolução ocorreu nos estudos do cosmos quando Joseph von Fraunhofer e Angelo Secchi foram os primeiros a utilizar a técnica de espectroscopia na observação de estrelas. Ao comparar espectros do Sol e de Sirius, perceberam que havia diferenças 
nas linhas "escuras" (de absorção), e Secchi, em 1865, começou a classificar as estrelas em tipos espectrais. Porém, a primeira versão da classificação espectral utilizada atualmente (O-B-A-F-G-K-M) foi desenvolvida por Annie Jump Cannon em meados de 1900 North et al., 1994).

Hoje em dia, é muito claro que estrelas possuem características muito distintas umas das outras e que nosso Universo possui estrelas de diferentes tipos, cores, massas, idades e composições químicas. Hoje somos capazes de estudar a evolução e a morte desses objetos, entender seus processos de formação e classificá-los em grupos a partir de outras características além do tipo espectral. Dessa necessidade de classificação e interpretação (de modo a encontrar características comuns em grupos de estrelas e entender suas histórias de formação), surge o conceito de populações estelares.

Populações estelares é um conceito originalmente proposto por Baade (1944), mas que é utilizado até hoje para nos ajudar a estudar a história evolutiva da nossa e de outras galáxias.

Neste trabalho, pretendemos explorar e otimizar um dos métodos de estudo de populações estelares, conhecido por ajuste espectral pixel-a-pixel (e.g. Cid Fernandes et al. 2005). Esse método é amplamente utilizado em análise de espectros de luz integrada, e é uma ferramenta importante para entender como aglomerados estelares e galáxias se formam e evoluem.

\subsection{Populações Estelares}

Há mais de 13 bilhões de anos, a maior parte da matéria bariônica do Universo era composta de átomos de Hidrogênio e Hélio, originários do Big Bang e matéria prima para a formação das primeiras estrelas. As estrelas originam-se do colapso gravitacional de uma nuvem de gás, cujo centro torna-se cada vez mais quente e denso até o ponto em que o Hidrogênio no núcleo passa a ser convertido em Hélio através dos ciclos próton-próton (pp) e pelo ciclo Carbono-Oxigênio-Nitrogênio (CNO), que predomina em temperaturas entre vinte e cinquenta milhões de Kelvin (Maciel, 1999).

A presença de metais pesados influencia os processos de formação desses objetos: uma nuvem molecular só é capaz de formar estrelas de baixa massa caso haja metais pesados em sua composição (Abel et al. 2002, Smith e Sigurdsson 2007). Assim, as primeiras estre- 
las, responsáveis pelo início do processo de reionização do Universo, eram extremamente massivas e extremamente pobres em metais. A esses objetos, damos o nome de População III (Bromm et al., 1999).

Tais estrelas, após o seu curto tempo de vida, explodiram em supernovas e iniciaram o processo de poluição do Universo: além da liberação para o meio interestelar dos elementos criados no interior das estrelas, a energia gerada dá origem ao processo de captura de neutrons, criando elementos ainda mais pesados (Norris et al., 2001). A presença desses metais nas nuvens moleculares, agora, possibilita a formação de estrelas de baixa massa (e portanto, de grande longevidade) algumas das quais observamos até hoje. Tais objetos (que chamamos de População II) são pobres em metais e datam de uma época muito próxima ao início do Universo, e nos proporcionam informações importantes sobre essa época cósmica (Baade 1944, Beers e Christlieb 2005).

Como resultado dessa produção de elementos durante os mais de 13 bilhões de anos desde a reionização, hoje as nuvens moleculares (presentes principalmente em galáxias disco e irregulares) são capazes de formar estrelas ricas em metais e com uma grande variedade de massas, tamanhos e cores. A esses objetos mais jovens, damos o nome de População I (Baade, 1944). Uma representação visual de tal processo de evolução das populações durante a história do Universo é mostrada na Figura 1.1.

Hoje em dia, com a melhora da qualidade dos instrumentos de observação, e consequentemente, dos dados, somos capazes de estudar populações estelares em maior detalhe. Portanto, as definições de Populações I, II e III tornaram-se simples demais. Atualmente, definimos uma população estelar como um grupo de estrelas que compartilha da mesma história evolutiva. Tal história está relacionada não só à época de nascimento dessas estrelas, mas também às condições nas quais esse processo ocorreu: taxa de formação estelar no ambiente, composição química e tamanho das nuvens moleculares afetarão significativamente uma população estelar nascida naquele meio. Assim, o estudo das composições químicas das estrelas é peça essencial na compreensão das populações estelares e da história da evolução do Universo.

O indicador de abundâncias químicas mais utilizado é a metalicidade. Em espectroscopia estelar, a medida mais comum para representar a metalicidade de um objeto é:

$$
[F e / H]=\log \left(\frac{N_{F e}}{N_{H}}\right)_{\star}-\log \left(\frac{N_{F e}}{N_{H}}\right)_{\odot}
$$




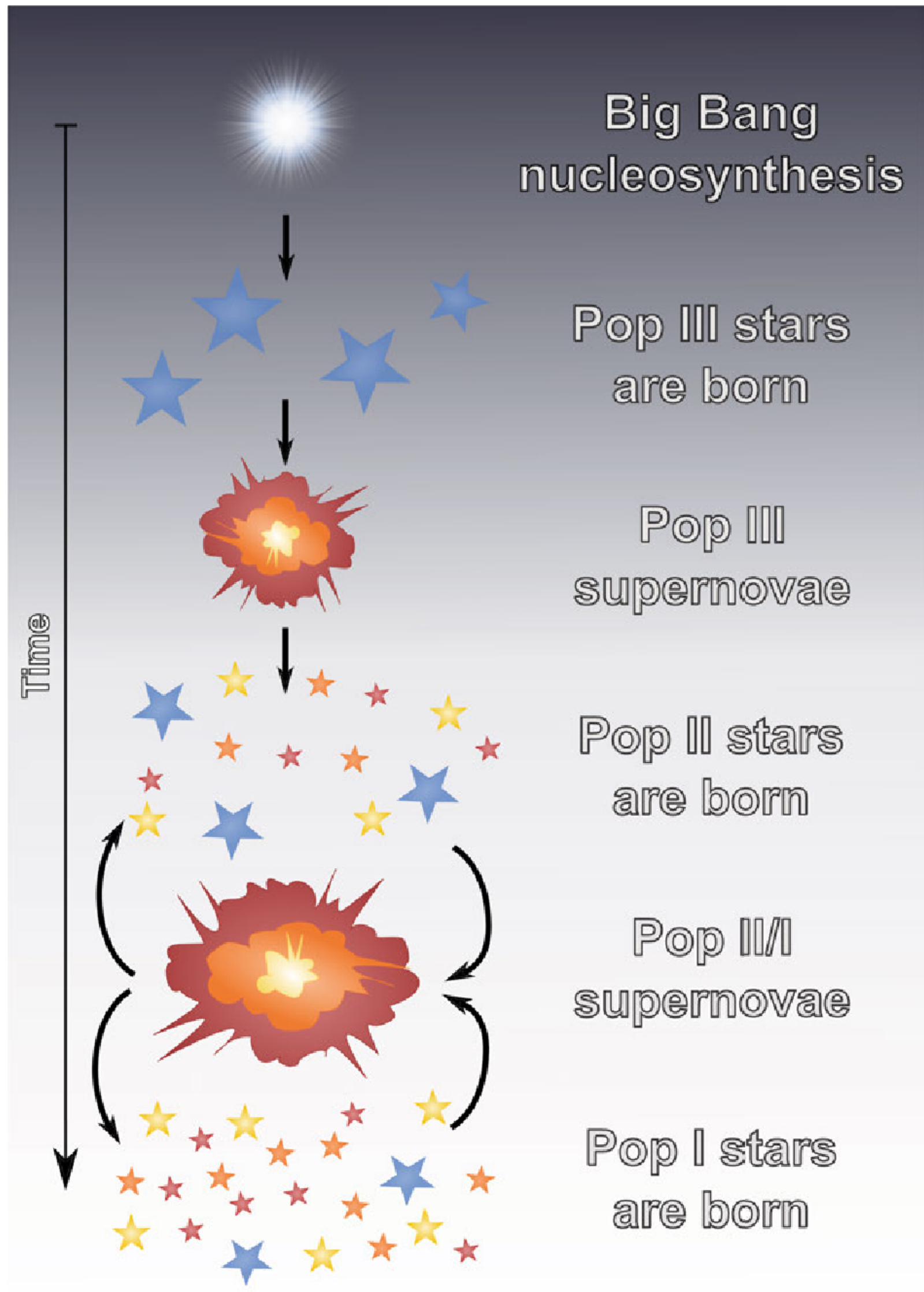

Figura 1.1: Representação visual (e simplificada) do surgimento das Populações I, II e III na história do Universo. Figura de Till (2017). 
onde $N_{F e}$ é o número de átomos de $\mathrm{Fe}, N_{H}$ é o número de átomos de $\mathrm{H}$ na superfície da estrela e os índices $\star$ e $\odot$ indicam o objeto estudado e o Sol, respectivamente. Logo, por definição, $[\mathrm{Fe} / \mathrm{H}]_{\odot}=0$.

Outro importante indicador da composição química das estrelas é a abundância de elementos- $\alpha$. Um elemento é assim denominado quando seus isótopos mais abundantes são múltiplos inteiros da massa do núcleo do Hélio (no caso, $\mathrm{Ne}, \mathrm{Mg}$, Si, S, Ar, Ca e Ti). A notação mais utilizada para representar tal indicador é:

$$
[\alpha / F e]=\log \left(\frac{N_{\alpha}}{N_{F e}}\right)_{\star}-\log \left(\frac{N_{\alpha}}{N_{F e}}\right)_{\odot}
$$

Elementos- $\alpha$ são criados majoritariamente durante explosões de supernova do tipo II (quando o núcleo de uma estrela massiva colapsa), numa escala de tempo de 3 a 30 milhões de anos (e.g. Pipino e Matteucci, 2009). Por causa da explosão, esses elementos são devolvidos ao meio interestelar e passam a fazer parte das nuvens moleculares. Na história evolutiva da nossa Galáxia, nuvens enriquecidas em elementos $\alpha$ foram as responsáveis pela origem das estrelas de População II e pelo padrão de abundância que observamos nesses objetos: enriquecidas com elementos- $\alpha$, porém ainda de baixa metalicidade $([\alpha / F e]>0$ e $[\mathrm{Fe} / \mathrm{H}]<0)$.

Com o tempo, estrelas de baixa massa que evoluíram em sistemas binários podem passar pelo processo que chamamos de supernova de tipo Ia: ocorre transferência de massa de uma gigante vermelha para sua companheira anã-branca, que ao ultrapassar o limite de massa de Chandrasekhar $\left(1.38 M_{\odot}\right)$ inicia o processo de fusão nuclear do Carbono, que libera energia o suficiente para fazer com que ela entre em processo de supernova (Khokhlov et al., 1993). Esse tipo de supernova é o grande responsável pela liberação de elementos do pico do Ferro (Cr, Mn, Fe, Co e Ni) para o meio interestelar. Tal processo leva uma escala de tempo muito maior que o enriquecimento por elementos- $\alpha$, de 30 milhões de anos até o tempo de Hubble (e.g. Pipino e Matteucci, 2009).

Com o aumento gradual da abundância de Ferro e as supernovas do tipo II sendo cada vez mais raras, esses objetos jovens tendem a mostrar valores de $[\alpha / \mathrm{Fe}]$ mais baixos. Isso é exemplificado na Figura 1.2 (Figura 1 de McWilliam 1997), que mostra a relação $[\alpha / \mathrm{Fe}]$ versus $[\mathrm{Fe} / \mathrm{H}]$ para a vizinhança solar, onde o eixo que indica $[\mathrm{Fe} / \mathrm{H}]$ também pode, a grosso modo, ser interpretado como indicador de tempo (baixo $[\mathrm{Fe} / \mathrm{H}]$ no início do Universo, alto $[\mathrm{Fe} / \mathrm{H}]$ agora). Logo, quando tratamos de sistemas complexos como galáxias espirais, por 


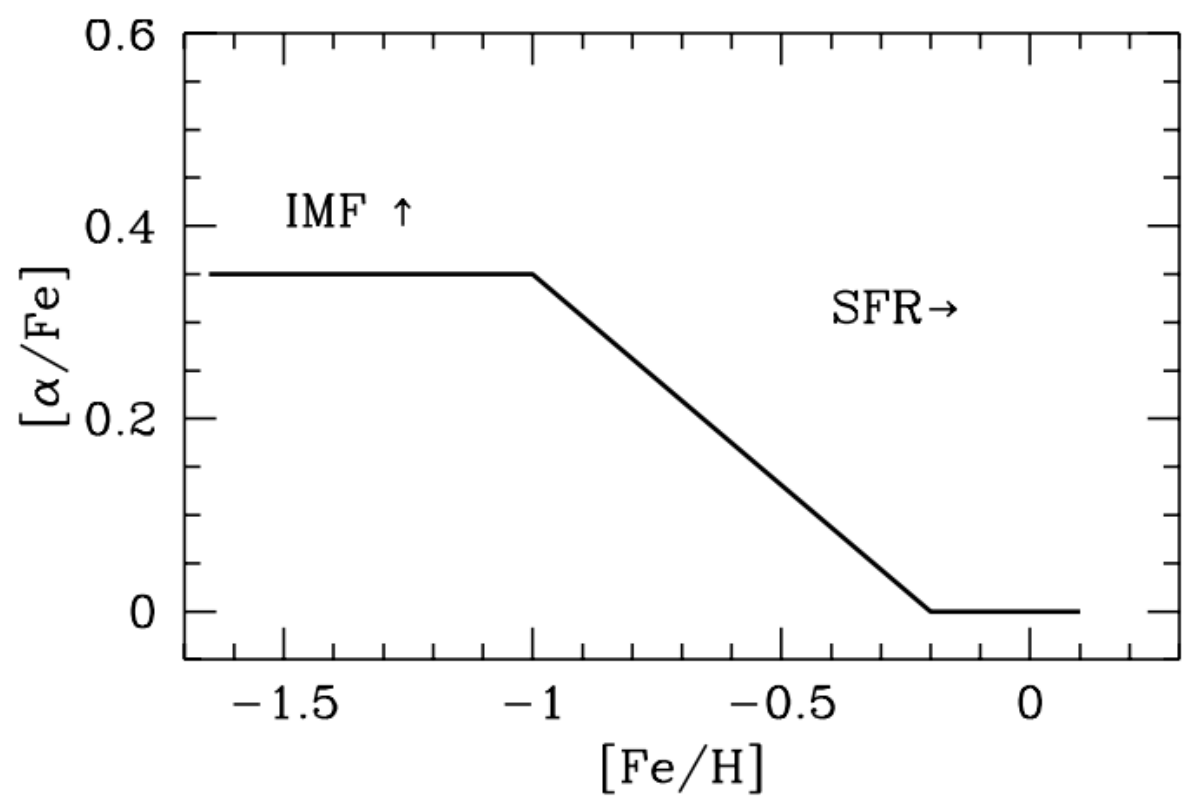

Figura 1.2: Figura 1 de McWilliam (1997). Esquema do comportamento das abundâncias de elementos- $\alpha$ com a metalicidade para a vizinhança solar. O "joelho" da curva indica o "momento"em que as supernovas do tipo Ia começaram a poluir o meio interestelar. A figura também mostra o comportamento esperado da curva devido à influência da Função de Massa Inicial (IMF) e da Taxa de Formação Estelar (SFR).

exemplo, esperamos encontrar uma diferença nos padrões de abundâncias químicas em seus componentes: estrelas do disco, lugar onde os processos de formação estelar ainda estão presentes, vão no geral conter estrelas de médio ou alto $[\mathrm{Fe} / \mathrm{H}]$ e baixo $[\alpha / \mathrm{Fe}]$, enquanto os aglomerados globulares (GCs, do inglês Globular Clusters) do halo da Via Láctea, via de regra, apresentarão estrelas mais antigas, pobres em metais e enriquecidas em elementos- $\alpha$.

\subsection{Aglomerados Globulares}

Diferentes sistemas abrigam diferentes configurações de populações estelares: em discos e bojos de galáxias espirais, por exemplo, esperamos encontrar uma grande distribuição de idades, enquanto aglomerados estelares são o exemplo que mais de aproxima de uma população estelar simples que encontramos na Natureza (Renzini, 1986).

Aglomerados globulares são conjuntos de estrelas com distribuição aproximadamente esférica ligados gravitacionalmente, com alta densidade estelar em suas regiões centrais (Figura 1.3) e tamanho típico de $\sim 15 \mathrm{pc}$ de raio. Eles contém as estrelas mais antigas já observadas e aparentemente estão presentes em todas as galáxias massivas (Harris, 1991). Na Via Láctea, eles estão distribuídos ao redor do centro da Galáxia (Figura 1.4), podendo 
ser observados no bojo (onde podem apresentar altos valores de metalicidade, e.g. Barbuy et al. 1998), no halo galáctico ou até mesmo no disco, quando suas órbitas cruzam esse sistema.

Na última década, o aumento da quantidade de observações desses objetos tem jogado luz em questões essenciais para a compreensão de seus processos de formação e evolução. Sabemos atualmente, por exemplo, que a ideia de que aglomerados globulares são equivalentes a populações estelares simples e sem variações de abundâncias químicas está ultrapassada (Bastian e Lardo, 2018, e.g.). Dados fotométricos de alta precisão tem mostrado que esses objetos podem apresentar Diagramas Cor-Magnitude (CMDs, do inglês ColorMagnitude Diagrams) complexos (apresentando, por exemplo, turn-offs de sequencia principal extendidos devido à presença de estrelas de alta rotação, Bastian e Lardo 2018). Além disso, análises de espectroscopia de alta resolução tem mostrado grandes variações em elementos como Carbono, Nitrogênio e Oxigênio (apesar de $\mathrm{C}+\mathrm{N}+\mathrm{O}$ tender a ser constante, Dickens et al. 1991) além de correlações (Na-N, Al-N) e anti-correlações (Na-O, Al-Mg) em estrelas de um mesmo aglomerado, associadas com a presença de multi-populações (Bastian e Lardo, 2018).

Assim, entender as populações estelares presentes em aglomerados globulares não é uma tarefa tão simples quanto acreditava-se anteriormente. Diferentes técnicas e abordagens podem ser usadas para tal propósito, e neste trabalho, tentaremos estudar as populações estelares desses objetos utilizando a técnica de ajuste espectral.

\subsection{Ajuste Espectral}

Os métodos de análise de populações estelares dependem do objeto a que se pretende estudar. Quando o alvo é a Via Láctea, somos capazes de resolver estrelas individuais, e podemos utilizar ajustes de isócronas em diagramas Cor-Magnitude ou calibrações asterosismológicas pra determinar idades (e.g. Dotter et al., 2010) de GCs, além de espectroscopia de alta resolução para determinar abundâncias químicas (e.g. Barbuy et al., 2018). Porém, para objetos mais distantes, resolver estrelas torna-se cada vez mais difícil, até o ponto em que temos que trabalhar com luz integrada.

Uma técnica amplamente utilizada por muitos anos no estudo de espectros de luz integrada foram os índices de Lick (e.g. Faber et al. 1985; Worthey 1994; Trager et al. 2000; 


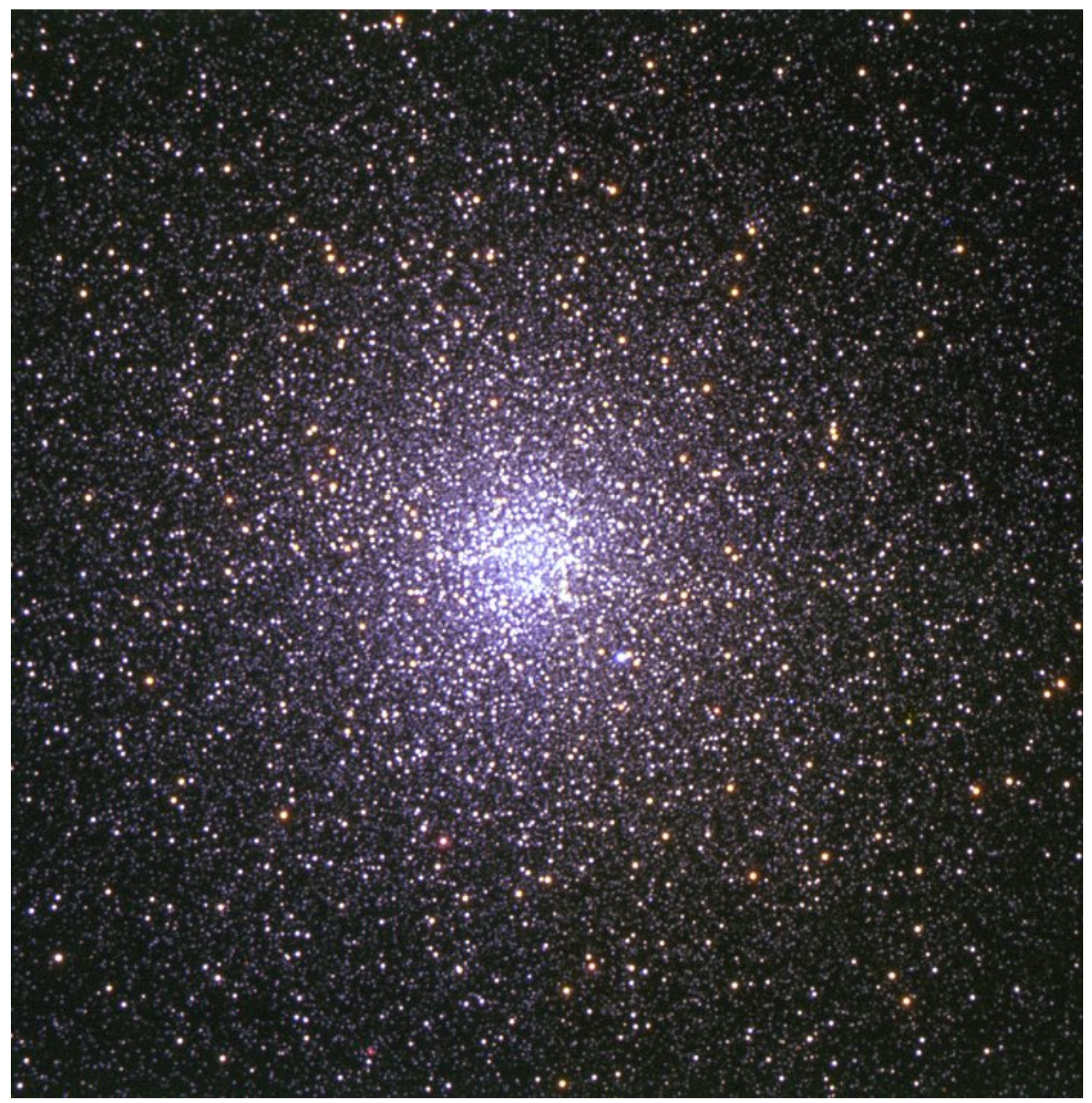

Figura 1.3: Aglomerado Globular NGC 104 (47Tuc). Composição de imagens das bandas U, R e uma banda estreita centrada em $485 \mathrm{~nm}$, observadas com o FORS1, no VLT. Créditos da imagem: ESO Science Archive, Rubina Kotak (ESO) e Henri Boffin (ESO). 


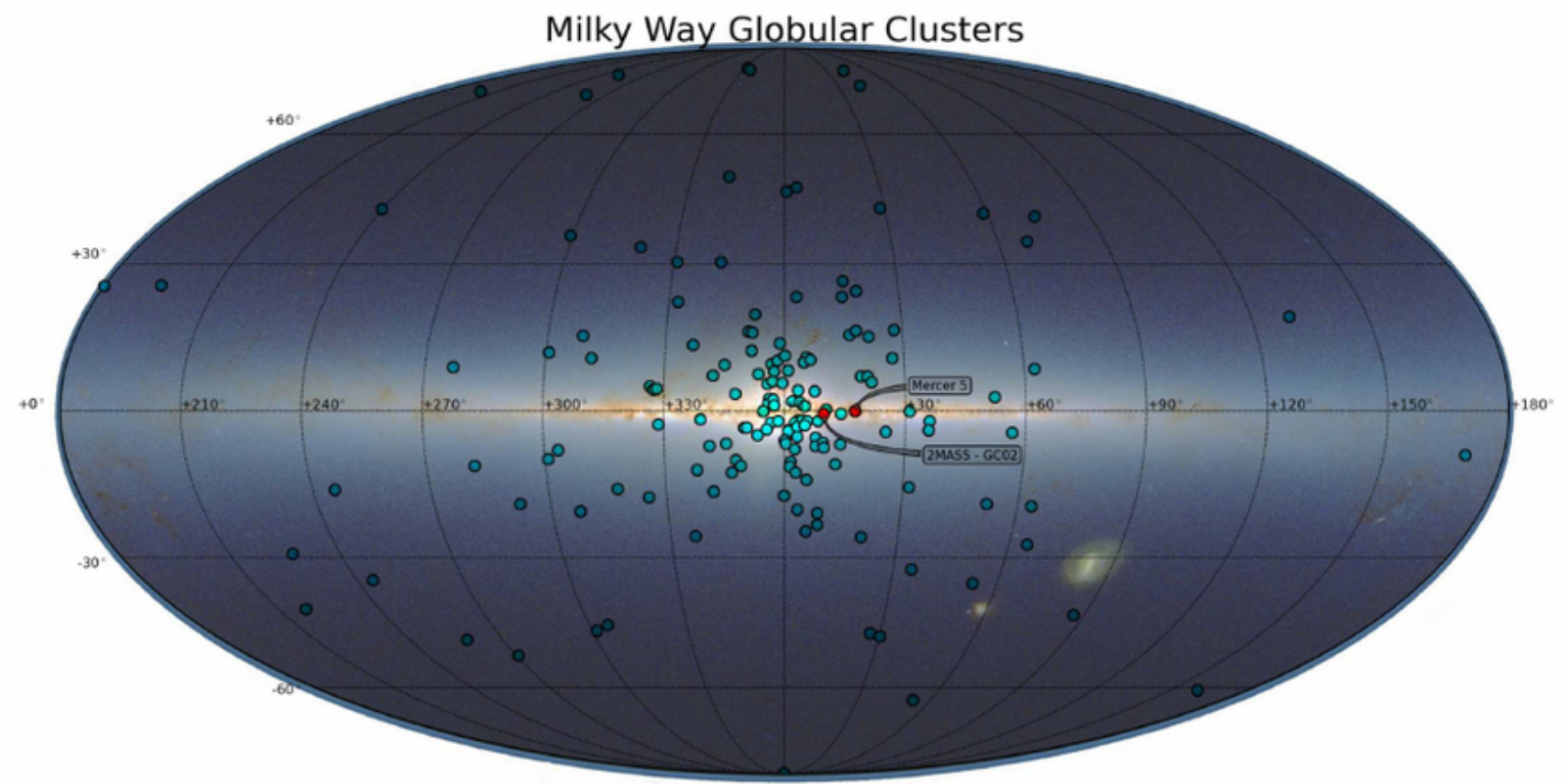

Figura 1.4: Distribuição dos aglomerados globulares observados da Via Láctea. Figura de Penaloza et al. (2015).

Thomas et al. 2003; Schiavon 2007), que consiste em medições de larguras equivalentes de linhas espectrais de absorção, além de bandas moleculares. Hoje em dia, com a maior disponibilidade de dados e o aumento dos recursos computacionais disponíveis, técnicas como o ajuste de SED (do inglês Spectral Energy Distribution, e.g. Walcher et al. 2011) e o ajuste espectral pixel-a-pixel (e.g. Cid Fernandes 2007; Koleva et al. 2008; Walcher et al. 2009; Conroy et al. 2014) têm sido mais comumente utilizadas.

Neste trabalho, focaremos no método de ajuste espectral, que parte da ideia de que podemos representar sistemas complexos (como galáxias, por exemplo) decompondo a informação integrada em sistemas mais simples, como estrelas individuais ou SSPs. Esse tipo de abordagem tem sido utilizado desde a década de 50, como por exemplo, nos trabalhos de Morgan (1956), Wood (1966) e Bica e Alloin (1987), entre outros.

Softwares de ajuste espectral, no geral, levam em conta toda a informação, $\lambda$-a- $\lambda$, contida no espectro de uma galáxia ou aglomerado estelar para encontrar, em meio a uma base de modelos de população estelar simples (SSP, do inglês Single Stellar Population), a combinação desses modelos que melhor representa o espectro observado. Assim, tanto o formato do contínuo quanto a informação contida nas linhas espectrais pode ser considerada. 
A escolha da base de SSPs a ser utilizada, portanto, é um aspecto crucial do método e pode influenciar significativamente os resultados. Uma grande variedade de modelos espectrais de SSP estão hoje disponíveis na literatura (e.g. Bruzual A. e Charlot 1993 , Leitherer et al. 1999, González Delgado et al. 2005, Coelho et al. 2007, Schiavon 2007, Maraston e Strömbäck 2011). Uma das bibliotecas mais utilizadas é a de Bruzual e Charlot (2003) (mais comumente chamada de BC03), que compreende modelos com idades de $10^{5}$ até $10^{10}$ anos e metalicidades $(\mathrm{Z})$ de 0.0001 até 0.1 . Além disso, as bibliotecas de Vazdekis et al. (2010) e Vazdekis et al. (2015) têm ganhado popularidade, sendo que a última contém informações sobre abundâncias de elementos- $\alpha$.

Utilizando espectros observados e uma base de SSPs, alguns códigos de ajuste espectral são capazes de estimar, além das populações estelares e da história evolutiva das galáxias ou aglomerados, parâmetros cinemáticos e de avermelhamento.

Fernandes e Delgado (2010) e Delgado e Fernandes (2010) apresentam uma análise de diversos aspectos técnicos do método e de seus resultados quando utilizado para ajustar aglomerados estelares. Os autores discutem, por exemplo, as discrepâncias entre ajustes de população simples e múltipla, o papel da escolha de diferentes modelos de SSPs, as mudanças que ajustes cinemáticos podem acarretar nos resultados, entre outros. Em Fernandes e Delgado (2010), concluem que o método retorna boas estimativas de idade, metalicidade e avermelhamento para grande parte dos espectros ajustados. Porém em alguns casos, os modelos não levam em conta uma população azul e velha, provavelmente associada a um ramo horizontal extendido. Em Delgado e Fernandes (2010), várias bibliotecas de modelos são usadas e todas elas retornam boas estimativas de idades, apesar de os valores resultantes serem sistematicamente maiores que as os valores ajustados utilizando CMDs. Já para metalicidade e avermelhamento, os resultados mostram uma variação maior quando diferentes bibliotecas de SSPs são utilizadas.

Apesar de amplamente utilizado, existem alguns aspectos do método que ainda não foram estudados em detalhe. Um deles é o impacto da escolha do intervalo de comprimento de onda utilizado no ajuste. Estudos na literatura tem mostrado resultados conflitantes:

- Fernandes e Delgado (2010) ajustaram aglomerados estelares no intervalo 3650$4600 \AA$, e mostram exemplos onde duas SSPs distintas retornam ajustes indistinguíveis. Porém, quando extrapolado para uma região mais vermelha (fora da região de cobertura dos espectros observados), é notável que as SSPs ajustadas mostram grandes 
diferenças tanto no formato do contínuo quanto na profundidade das linhas de absorção. Os autores afirmam que uma cobertura espectral maior seria necessária para distinguir os melhores ajustes em casos como esses.

- Em Koleva et al. (2008), os autores utilizaram dois algoritmos diferentes - STECKMAP (Ocvirk et al. 2006) e NBURSTS (Chilingarian et al., 2007) - e três bibliotecas de SSPs - ELODIE 3.1 (Prugniel et al., 2007), BC03 (Bruzual e Charlot 2003 e MILES (Vazdekis 1999, Vazdekis et al. 2003) - para ajustar espectros de aglomerados globulares de Schiavon et al. (2005) e o espectro integrado de M67 de Schiavon et al. (2004). Os ajustes foram feitos em 4000-5700 $\AA$ e resultaram em boas estimativas de idade e metalicidade para 36 de 41 aglomerados. Mas eles argumentam terem evitado as regiões mais azuis do espectro, ou as idades resultantes mostrariam valores mais baixos.

- Walcher et al. (2009) utilizaram o algoritmo SEDFIT (Walcher et al., 2006) para ajustar espectros de NGC6528 e NGC6533 de Schiavon et al. (2005). Os autores testaram cinco diferentes regiões de comprimento de onda e mostram que o intervalo 4828-5364 A resulta em valores de idade, $[\mathrm{Fe} / \mathrm{H}]$ e $[\alpha / \mathrm{Fe}]$ mais próximos dos valores de literatura, enquanto que os demais intervalos parecem ser mais afetados pela degenerescência idade-metalicidade.

- Em Cezario et al. (2013), foram utilizados dois diferentes conjuntos de espectros de aglomerados globulares: GCs de M31 de Alves-Brito et al. (2009) e GCs galácticos de Schiavon et al. (2005). Com o algoritmo ULySS (Koleva et al., 2009) e SSPs de Vazdekis et al. (2010), os autores testaram quatro diferentes regiões espectrais com

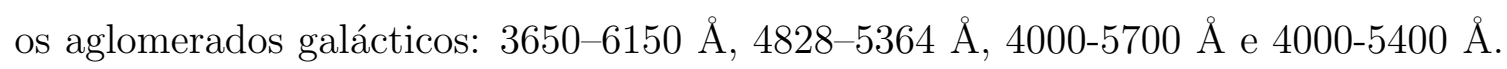
Eles concluíram que tal escolha tem pouca influência no cálculo das metalicidades, enquanto as idades podem mostrar variações significativas: um terço dos espectros foram ajustados com idades intermediárias em alguns casos. Eles argumentam que o intervalo 4000-5400 A é o que melhor reproduz idades provenientes de ajustes de isócronas, e em seguida aplicaram a técnica para os aglomerados de M31.

Logo, a utilização do método de ajuste espectral não é trivial e análises a respeito de sua usabilidade e confiabilidade são necessárias. 


\subsection{Objetivos}

Neste trabalho, pretendemos explorar em mais detalhe a dependência dos resultados de ajustes espectrais com o intervalo espectral utilizado. Assim, esperamos encontrar uma configuração que permita a um usuário utilizar ajustes espectrais com maior confiabilidade. Desse modo, nos propomos a prover um método simples de extrair parâmetros de populações estelares de luz integrada de forma consistente, uma vez escolhidos um algoritmo e uma biblioteca de modelos para os ajustes.

Abordamos essa questão utilizando espectros integrados de aglomerados globulares, por serem objetos com maior disponibilidade de valores de referência na literatura para os parâmetros que almejamos estudar (avermelhamento, idade, metalicidade e abundância de elementos- $\alpha$ ). Assim, esperamos que este trabalho traga contribuições para os estudos de populações estelares e de luz integrada, suas propriedades e métodos de análise. Em seguida, aplicamos o que aprendemos ao estudo de uma amostra de galáxias do Universo Local.

Este texto é organizado da seguinte maneira: o capítulo 2 apresenta as bases de dados de aglomerados e galáxias utilizados; o capítulo 3 apresenta a metodologia, incluindo nossas bases de modelos e algoritmos utilizados; o capítulo 4 discute os resultados; o capítulo 5 apresenta uma aplicação feita com espectros de bojos de galáxias do SDSS; e o capítulo 6 sumariza nossas conclusões. 
Capítulo 2

\section{Bases de dados}

De modo a investigar a eficiência da técnica de ajuste espectral na recuperação de parâmetros de populações estelares, optamos por trabalhar com espectros de luz integrada de aglomerados globulares. Tais objetos, por estarem mais próximos, facilitam a obtenção de dados de alta qualidade: é possível resolver estrelas, obter espectros de alta resolução e construir diagramas cor-magnitude, por exemplo. Graças a isso, a literatura proporciona estudos detalhados de idades e abundâncias químicas desses objetos, que podem ser usados como referência.

Além disso, também aplicamos o método numa amostra de espectros de luz integrada de bojos de galáxias do Universo local, de modo a estudar as populações estelares desses sistemas.

Na Seção 2.1, apresentamos as bibliotecas de luz integrada de aglomerados globulares utilizadas nesse trabalho, e a compilação dos dados da literatura sobre tais aglomerados. A Seção 2.2 apresenta os dados de luz integrada e de morfologia dos bojos de galáxias estudados.

\subsection{Bibliotecas de luz integrada de aglomerados globulares}

Grande parte deste trabalho foi feito utilizando dados espectroscópicos de luz integrada da biblioteca WAGGS (WiFes Atlas of Galactic Globular clusters Spectra, Usher et al. 2017), que observou espectros de 64 aglomerados da Via Láctea e 22 de galáxias satélites (Grande e Pequena Nuvens de Magalhães e Fornax). A Figura 2.1 mostra as distribuições de idade por metalicidade (painel a) e $[\alpha / \mathrm{Fe}]$ por metalicidade (painel b) dos aglomerados da biblioteca WAGGS. 

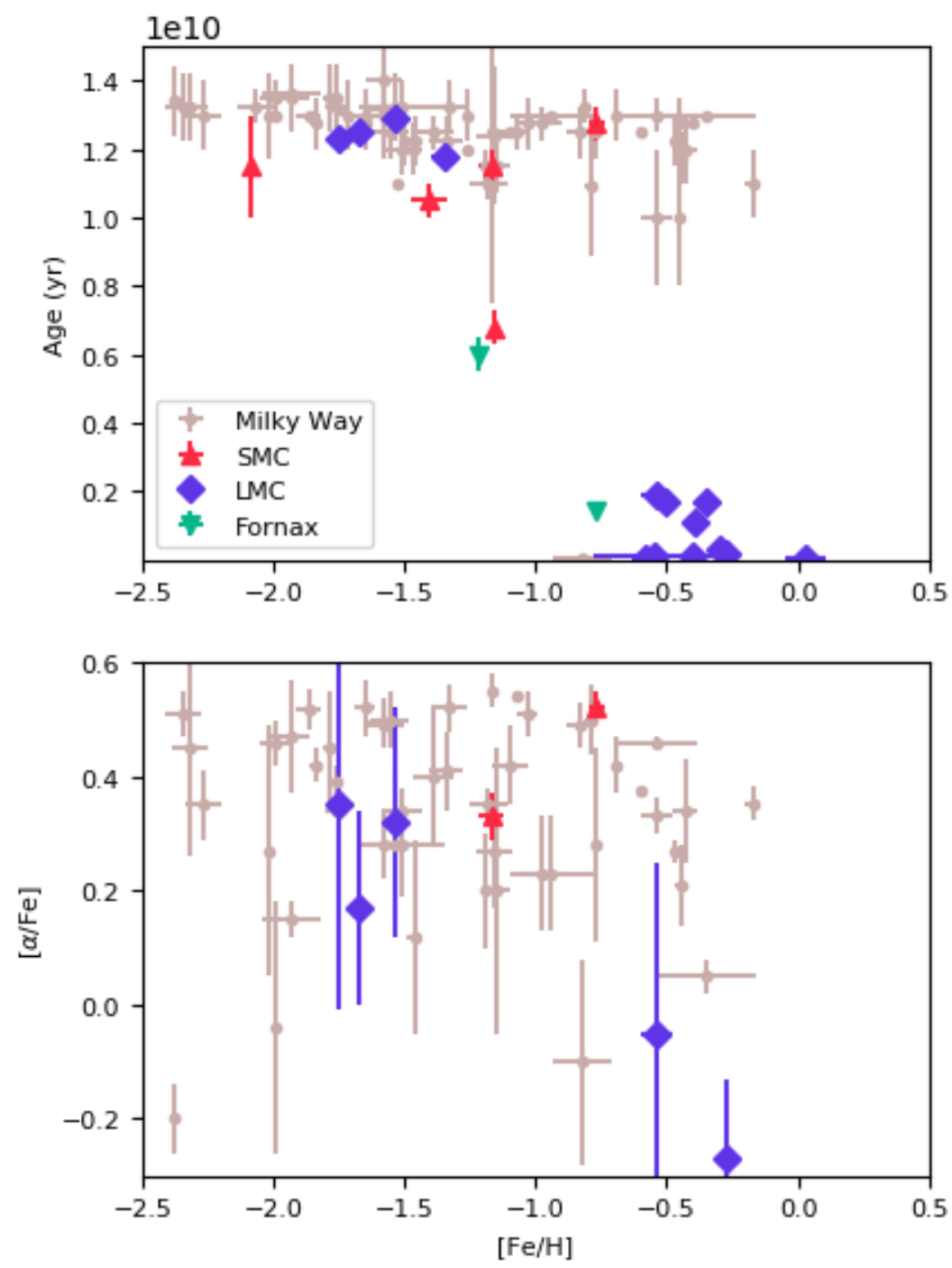

Figura 2.1: Distribuições de idade e abundâncias químicas dos aglomerados globulares presentes na biblioteca WAGGS. O painel superior mostra idades versus $[\mathrm{Fe} / \mathrm{H}]$, e o painel inferior mostra $[\alpha / \mathrm{Fe}]$ versus $[\mathrm{Fe} / \mathrm{H}]$. Galáxias hospedeiras estão indicadas por diferentes cores e símbolos. Crédito: Gonçalves et al. (submetido) 
Tabela 2.1 - Intervalos de comprimento de onda dos espectros da biblioteca WAGGS

\begin{tabular}{ll}
\hline Nome & Intervalo $(\AA)$ \\
\hline$U 7000$ & $3270.5-4350.0$ \\
$B 7000$ & $4170.0-5540.0$ \\
$R 7000$ & $5280.0-7020.0$ \\
$I 7000$ & $6800.0-9050.0$ \\
\hline
\end{tabular}

A obtenção dos dados foi feita utilizando o espectrógrafo WiFeS (Dopita et al. 2007. 2010) no telescópio ANU (2.3m) no Observatório de Siding Spring, Coonabarabran, Nova Gales do Sul, Austrália. WiFeS divide a imagem em 25 partes de 1 por 38 arco-segundos cada (logo, o campo de visão total é de 25 por 38 arco-segundos). A luz de cada imagem é dividida entre dois braços (azul e vermelho), tendo cada braço uma rede holográfica que dispersa cada "fatia" como um espectro de fenda longa em um CCD de 4096 por 4096 pixels. Foram utilizadas duas configurações de grade, resultando em quatro espectros com diferentes coberturas espectrais para cada objeto (Tabela 2.1). As configurações resultam numa resolução de $\lambda / \delta \lambda \sim 6800$. Os espectros de erro foram calculados usando a pipeline PYWIFES (Childress et al., 2014), e os dados estão disponíveis publicamente no site do projeto WAGGST,

Neste trabalho, os espectros com a cobertura I7000 não foram utilizados por estarem fora da cobertura espectral dos modelos de população estelar simples que utilizamos (que serão descritos na Seção 3.1). Os valores medianos de sinal-ruído dos demais grupos são 12, 61 e 124 (U7000, B7000 e R7000, respectivamente), e as distribuições são mostradas na Figura 2.3. Segundo Christopher Usher (comunicação privada), a pipeline de redução dos dados não é perfeita, e os valores de erro podem estar superestimados para SNRs baixos e subestimados para altos SNRs. Em particular, para valores baixos de SNR, a principal fonte de incerteza são os efeitos estocásticos dada a massa estelar finita dentro do campo de visão observado.

A Tabela 1 de Usher et al. (2017) apresenta uma lista de todos os objetos da biblioteca WAGGS. Utilizando tal tabela como ponto de partida, fizemos uma extensa busca na literatura por valores de referência.

Para as idades, demos prioridade ao trabalho de Dotter et al. (2010), que mediu as ida-

\footnotetext{
1 http://www.astro.1jmu.ac.uk/ astcushe/waggs/
} 
des de 39 dos 86 aglomerados da biblioteca WAGGS usando as isócronas de Dotter et al. (2007), adotando as escalas de metalicidade de Zinn e West (1984). Para os demais aglomerados, valores de outros trabalhos foram tomados como referência, conforme a coluna 9 da Tabela 2.2.

Para as abundâncias químicas, priorizamos trabalhos de espectroscopia de alta resolução que disponibilizassem valores tanto de $[\mathrm{Fe} / \mathrm{H}]$ quanto de $[\mathrm{Mg} / \mathrm{Fe}]$ (que adotamos como valores para $[\alpha / \mathrm{Fe}])$. Coletamos tais informações de diversas fontes distintas, como consta na coluna 7 da Tabela 2.2 .

Para algumas análises adicionais, também utilizamos dados espectroscópicos de luz integrada de aglomerados globulares da biblioteca de Schiavon et al. (2005), que contém 40 aglomerados globulares da Via Láctea.

Os dados foram obtidos utilizando o espectrógrafo Ritchey-Chretien, no telescópio Blanco (4.0m), no Observatório Cerro Tololo Inter-American em Vale de Elqui, Coquimbo, Chile. De modo a obter espectros de luz integrada representativos de toda a extensão dos aglomerados, as observações foram feitas movendo a posição da fenda em toda a extensão da região central do objeto. Os dados possuem uma cobertura espectral de $3350-6430 \AA$ resolução de $\sim 3.1 \AA$ (FWHM), e estão disponíveis no site do National Optical Astronomical Observatory $\left.\right|^{2}$.

A Figura 2.2 ilustra espectros de três dos aglomerados comuns nas duas bibliotecas sobrepostos.

\footnotetext{
2 http://www.noao.edu/ggclib
} 

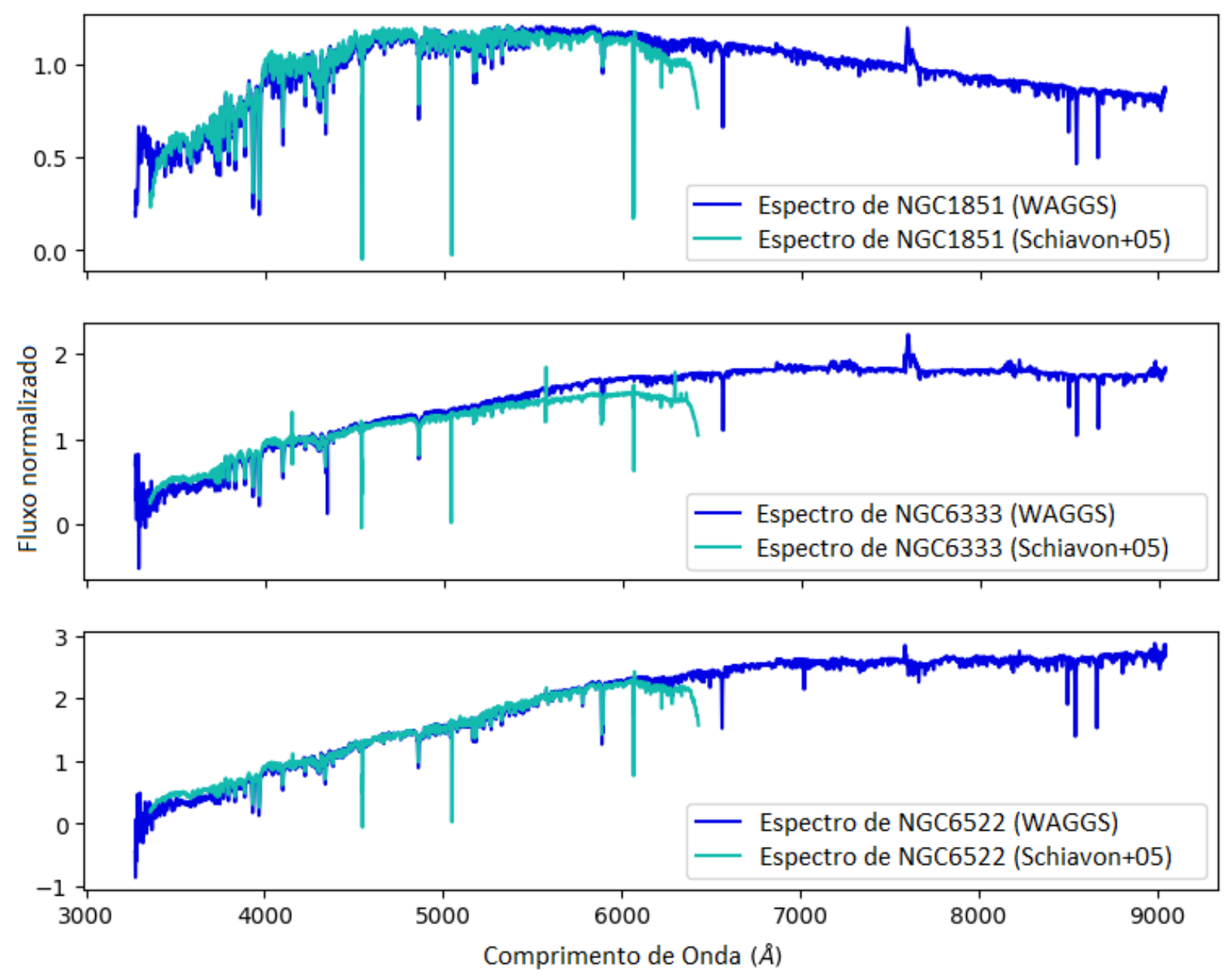

Figura 2.2: Espectros dos aglomerados NGC1851, NGC6333 e NGC6522 das bibliotecas WAGGS (azul) e Schiavon et al. 2005 (verde) sobrepostos. 


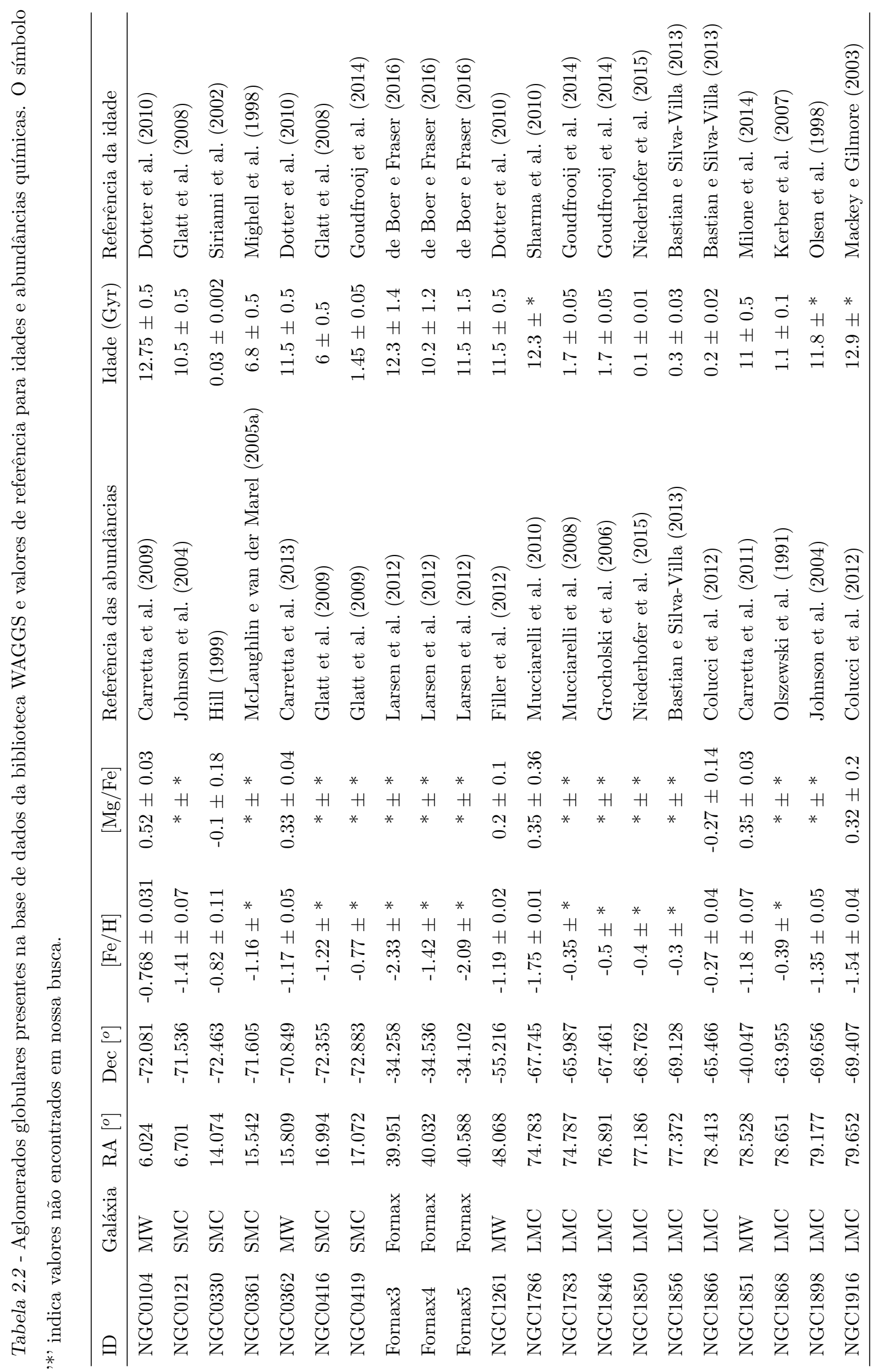




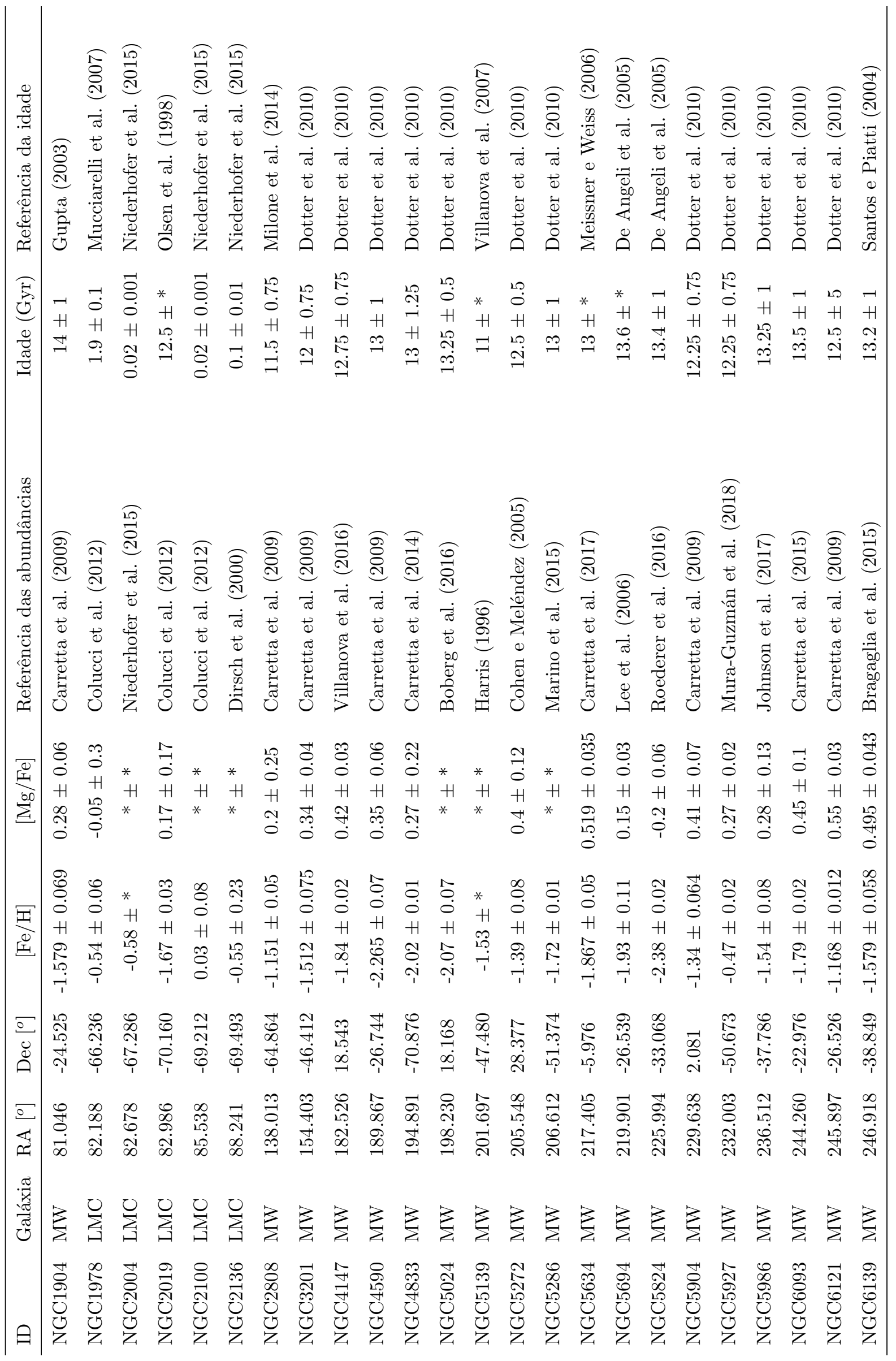




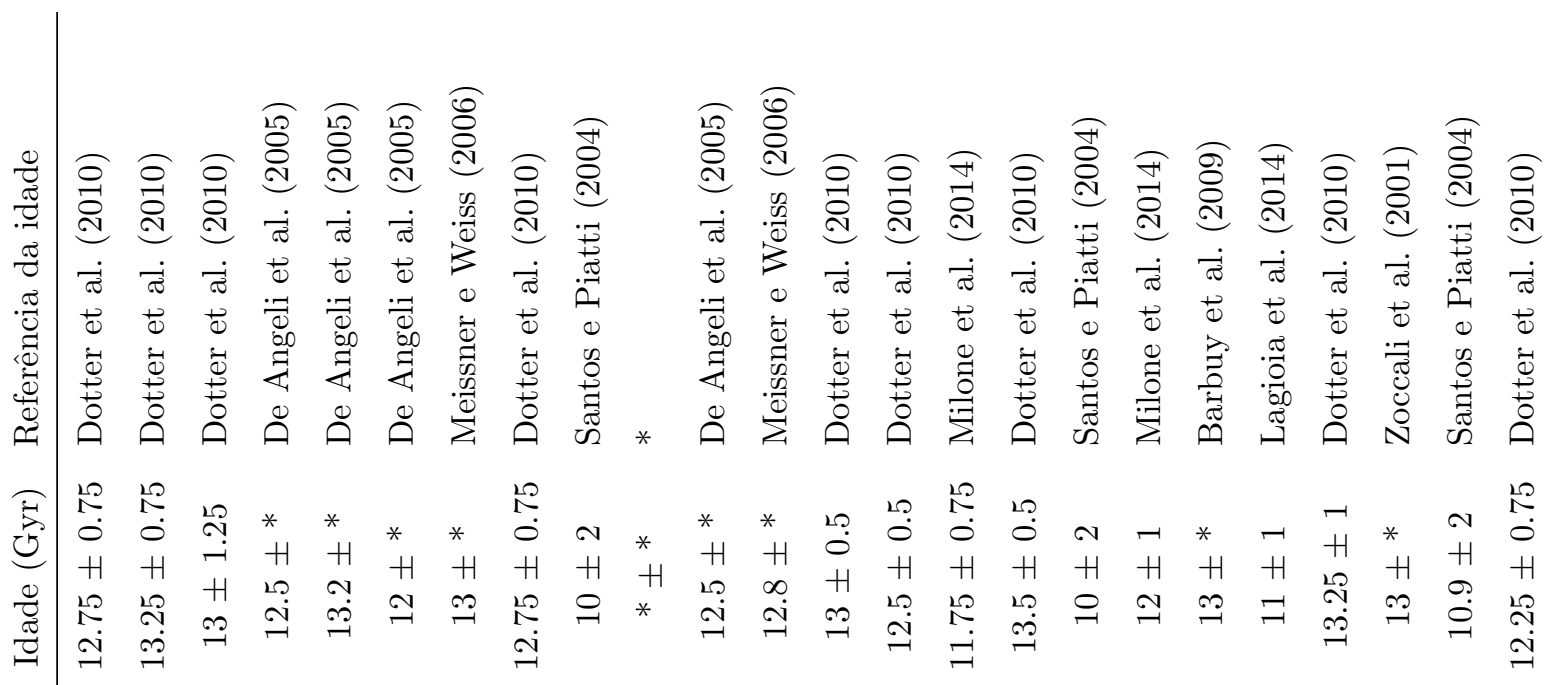

:

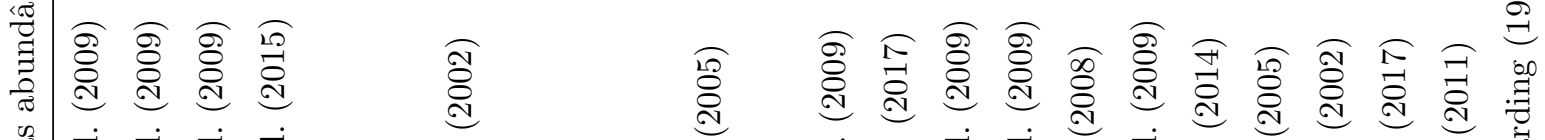

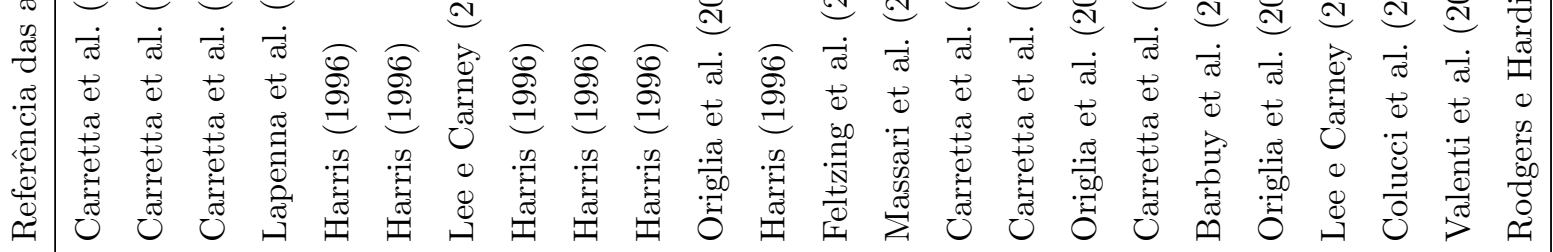

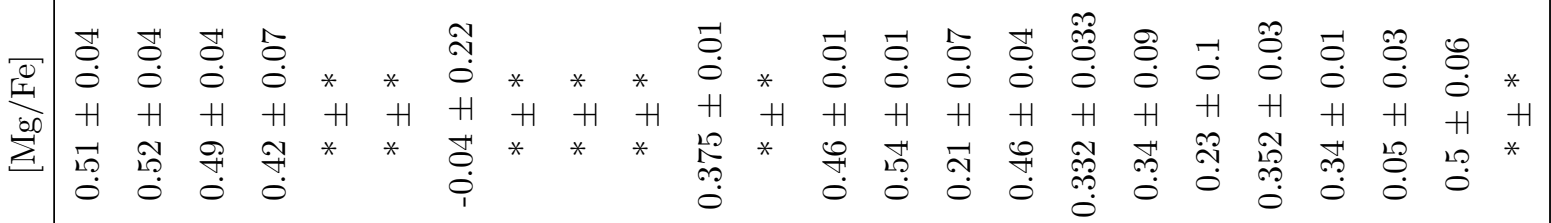

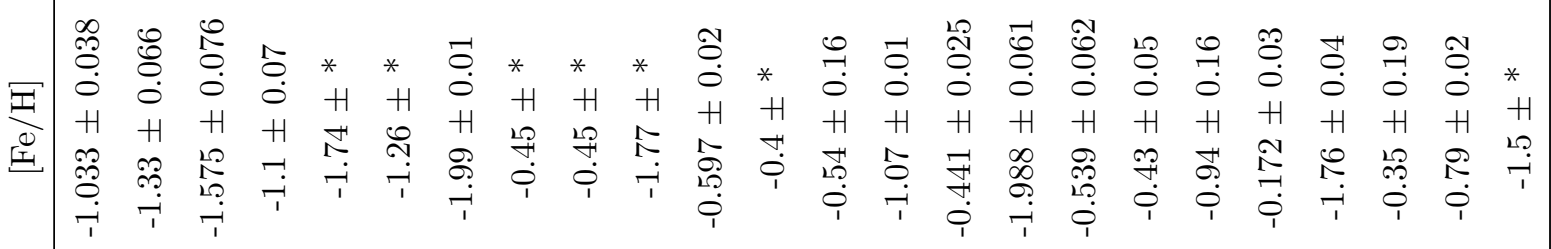
ஏ

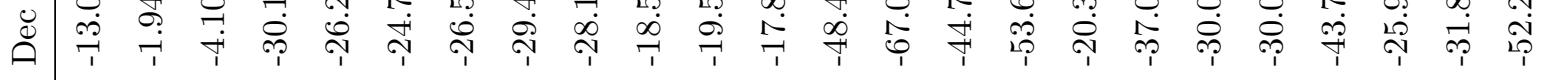

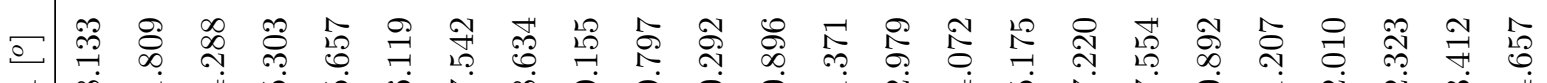

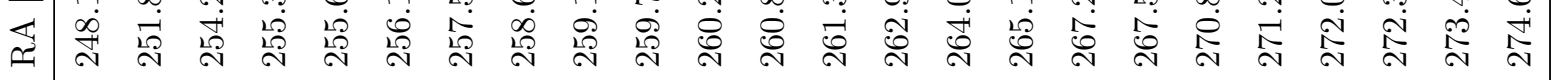

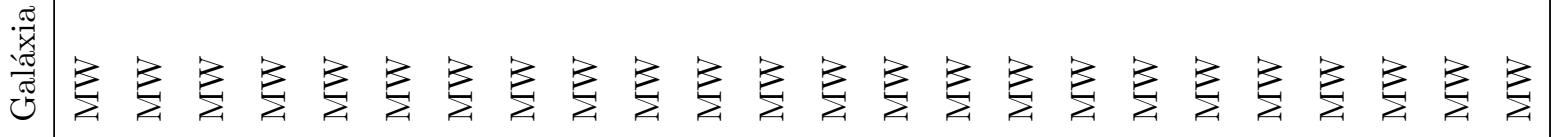

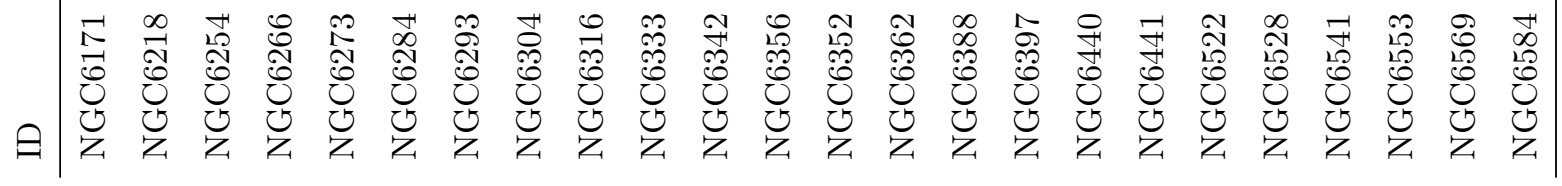




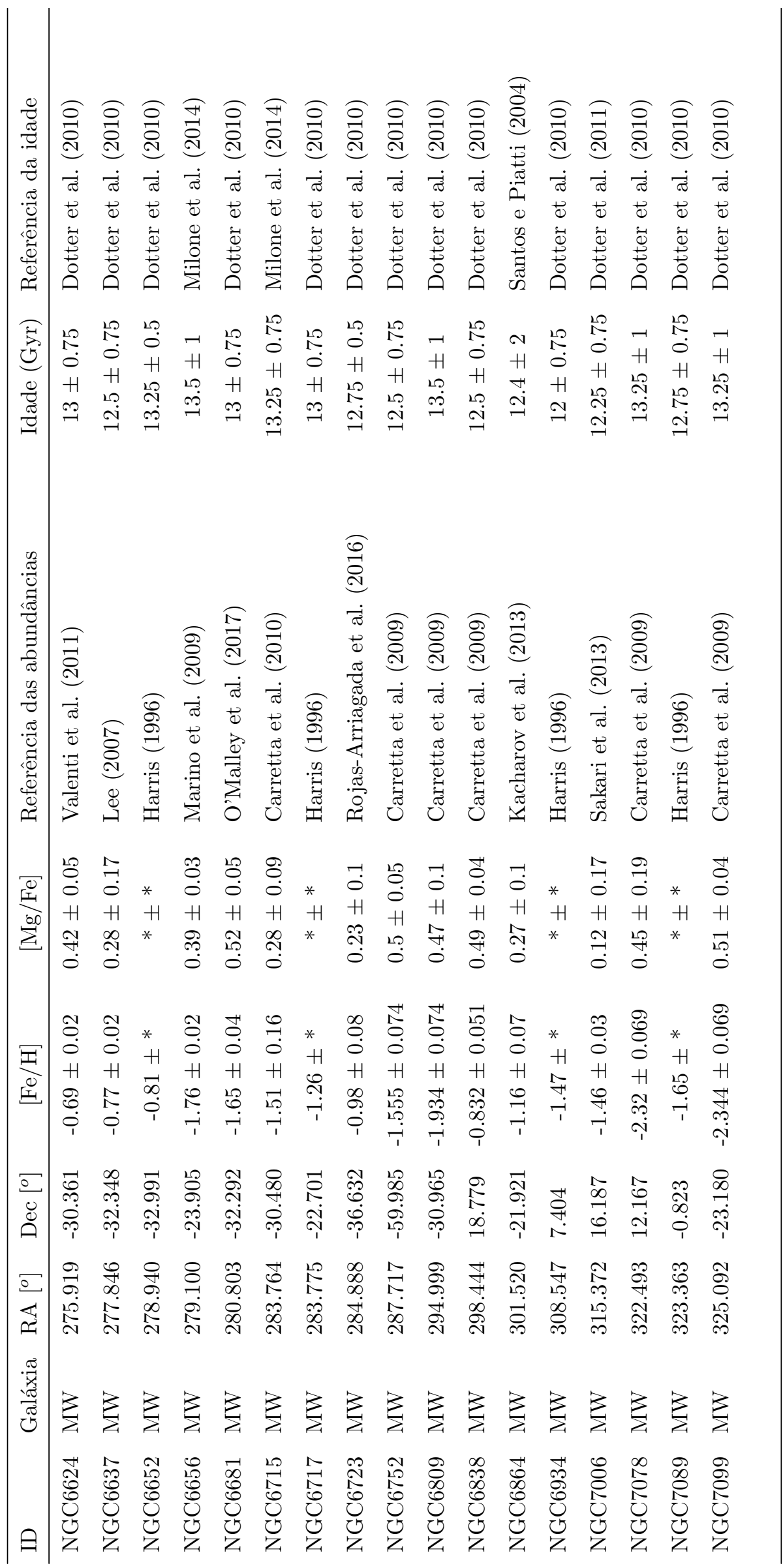



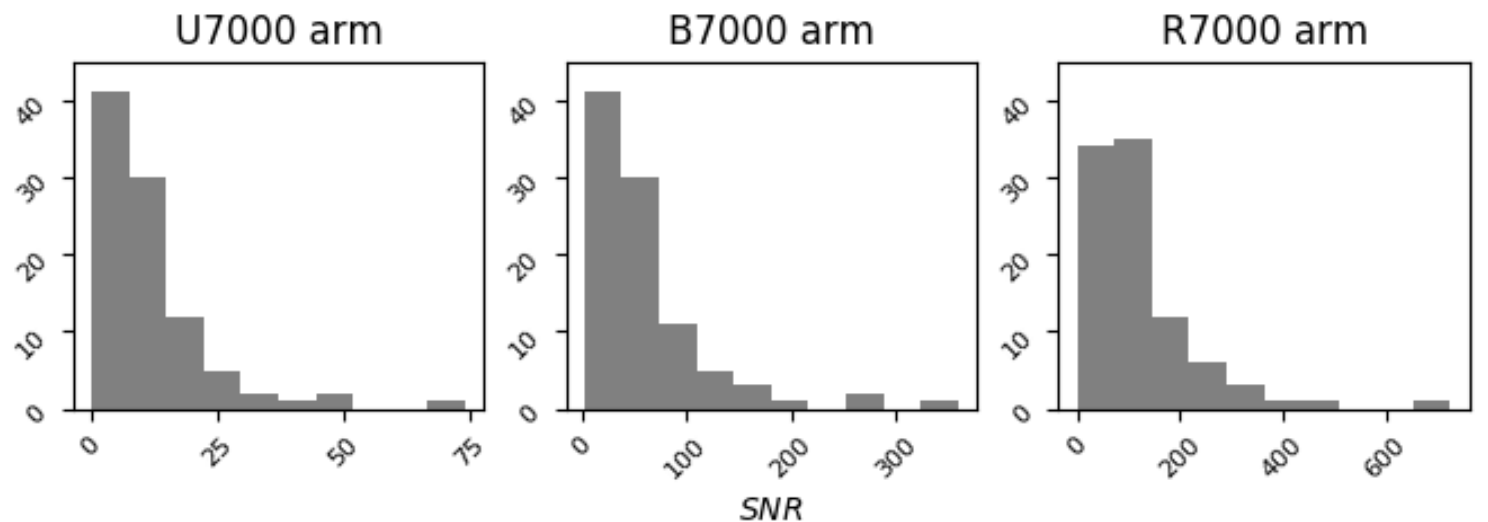

Figura 2.3: Distribuição de valores de sinal-ruído (SNRs) dos espectros da biblioteca WAGGS. Os três painéis mostram histogramas de SNR para os espectros U7000, B7000 e R7000, respectivamente. É notável que os espectros U7000 possuem os valores mais baixos da biblioteca. Crédito: Gonçalves et al. (submetido)

\subsection{Uma amostra de bojos galácticos do Universo próximo: espectroscopia e parâmetros morfológicos}

Neste trabalho, estudamos um conjunto de aproximadamente mil galáxias do Universo local, classificadas morfologicamente como elípticas, espirais com bojo clássico, espirais com pseudo-bojo ou espirais sem bojo, sendo que os bojos podem ou não conter barras. Uma subamostra dos objetos é apresentada na Figura 2.5, e a Figura 2.6 mostra o número de galáxias por morfologia, também divididas em galáxias AGN ou não-AGN.

Utilizamos os parâmetros morfológicos desses objetos apresentados em Gadotti (2009). O autor faz uma decomposição 2D de imagens de 946 galáxias do Sloan Digital Sky Survey Data Release 2 (SDSS DR2, Abazajian et al. 2004), utilizando as bandas $g, r$ e $i$, de modo a inferir parâmetros estruturais e massa estelar desses sistemas. Assim, são separados e caracterizados componentes como bojos clássicos, pseudo-bojos, barras, discos e galáxias elípticas. Tal decomposição foi feita utilizando o código BUDDA (de Souza et al., 2004). A Tabela 2.3 apresenta os parâmetros morfológicos disponibilizados pelo autor.

Os espectros das galáxias, também provindos do SDSS DR2, possuem uma cobertura espectral de 3200-9200 A, com resolução $R=2000$. A Figura 2.4 mostra a distribuição de sinal-ruído desses espectros.

Coelho e Gadotti (2011) fizeram uma análise de um subgrupo dessa amostra (excluindo as galáxias elípticas e espirais sem bojo), de modo a analisar a influência das barras nos processos de formação estelar nos bojos. Os autores concluíram que a distribuição de 

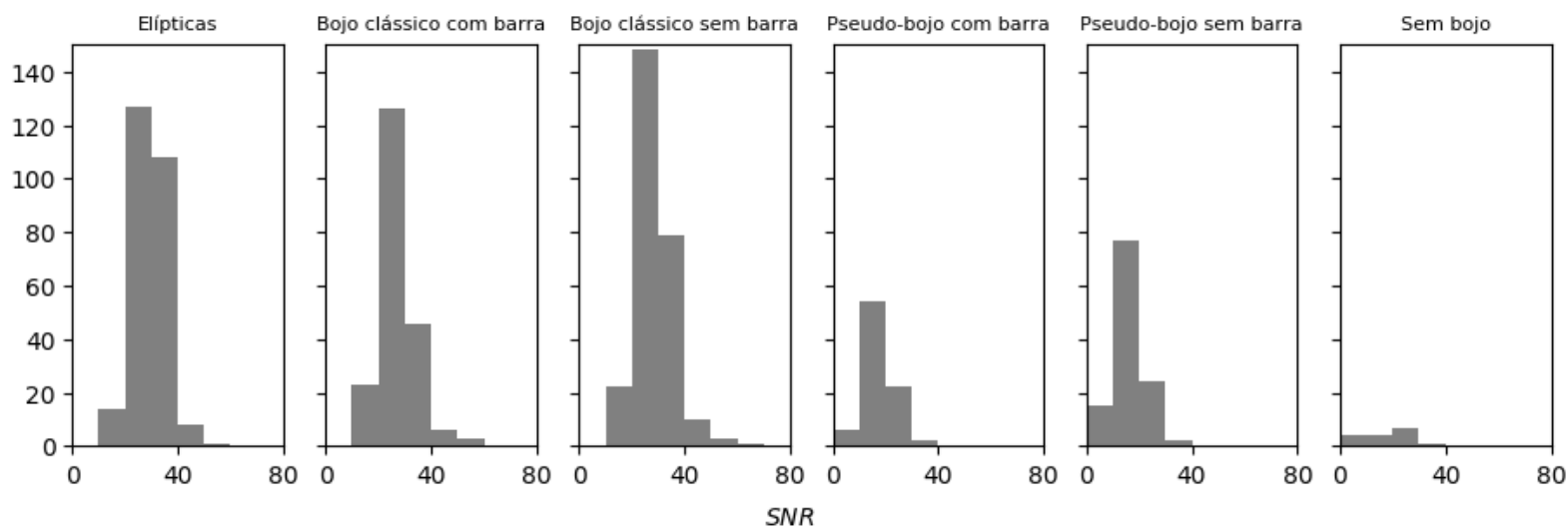

Figura 2.4: Distribuição de valores de sinal-ruído (SNRs) dos espectros de bojos de galáxias utilizados neste trabalho. Os painéis mostram histogramas de SNR para diferentes morfologias.

idades em bojos de galáxias barradas apresenta um excesso de populações mais jovens que 4 Gyr. Ao dividir a amostra entre galáxias AGN e não-AGN, as distribuições mostram que tal efeito acontece em galáxias de núcleo inativo, e que desaparece em AGNs. Esse resultado é uma evidência de que as barras tem um papel importante no deslocamente do gás até a região central das galáxias, podendo assim aumentar a taxa de formação estelar nos bojos, conforme expectativas teóricas (e.g. Athanassoula 2005). Além disso, esse resultado também mostra que barras podem ter um papel importante no aumento da quantidade de gás disponível para alimentar um AGN, o que poderia frear o processo de formação estelar (Schawinski et al., 2007).

Neste trabalho nós re-analisamos essa amostra, incluindo elípticas e galáxias disco sem bojo, utilizando um conjunto mais recente de modelos de população estelar. 


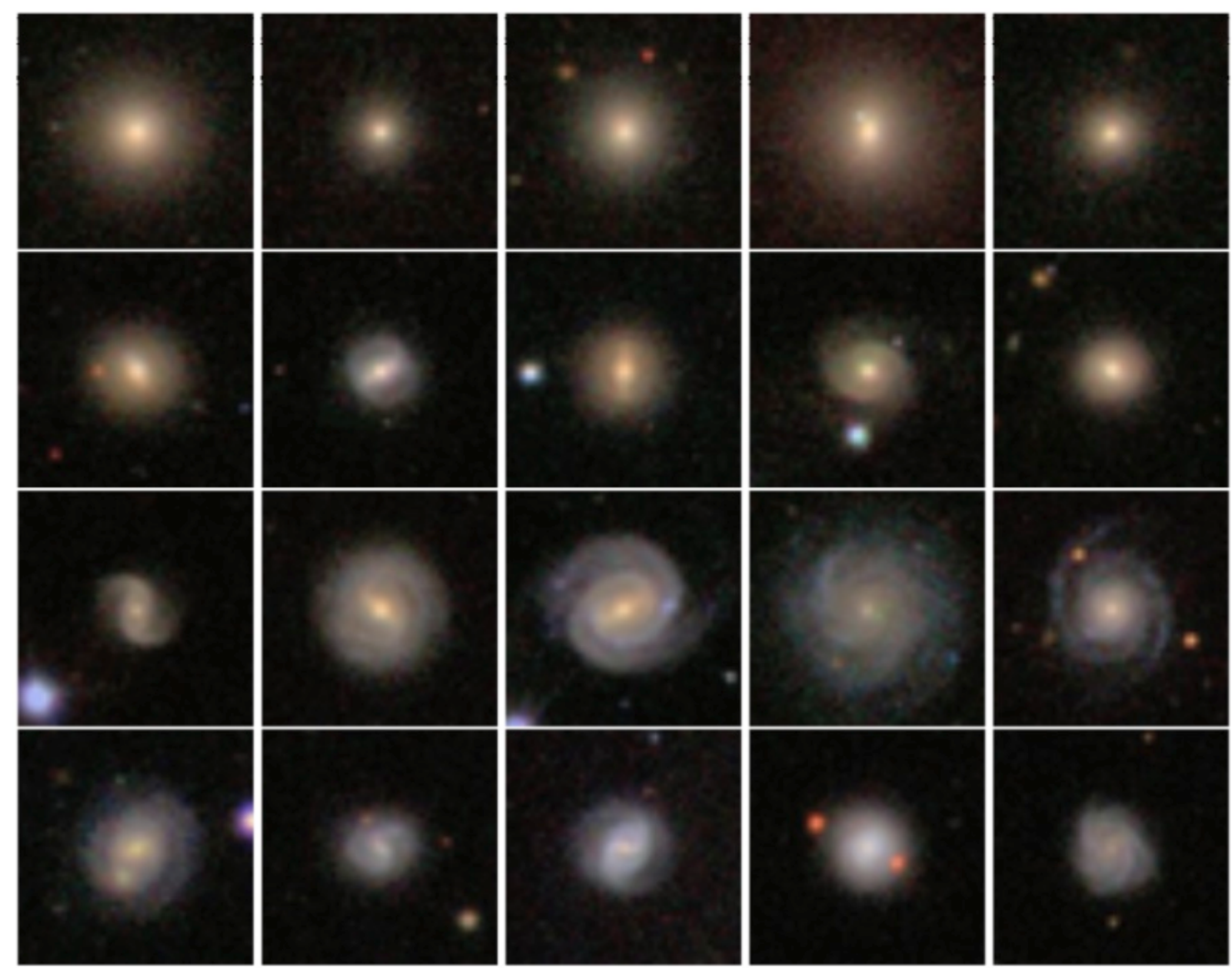

Figura 2.5: Exemplos de galáxias da amostra de Gadotti (2009). As linhas ilustram, de cima para baixo: galáxias elípticas, galáxias com bojos clássicos, galáxias com pseudo-bojos e galáxias sem bojo. Para as três últimas linhas, as três primeiras colunas da esquerda ilustram galáxias com barra. Crédito: Gadotti $(2009)$ 
Tabela 2.3 - Parâmetros morfológicos e estruturais disponíveis para as 946 galáxias do conjunto de Gadotti (2009).

\begin{tabular}{ll}
\hline Nome & Descrição \\
\hline$\mu_{0}$ & Brilho superficial central do disco \\
$h$ & Escala de largura do disco \\
$\mu_{e}$ & Brilho superficial central do bojo \\
$r_{e}$ & Raio efetivo do bojo \\
$n$ & Perfil de Sérsic do bojo \\
$\mu_{e, b a r}$ & Brilho superficial central da barra \\
$r_{e, b a r}$ & Raio efetivo da barra \\
$\epsilon_{\text {bar }}$ & Elipsidade da barra \\
$n_{\text {bar }}$ & Perfil de Sérsic da barra \\
$L_{b a r}$ & Eixo semi-maior da barra \\
$c$ & Boxyness da barra \\
$B / T$ & Proporção de brilho bojo/total \\
$D / T$ & Proporção de brilho disco/total \\
Bar $/ T$ & Proporção de brilho barra/total \\
$\chi^{2}$ & $\chi^{2}$ do ajuste \\
SEEING $H W H M$ & Seeing da observação \\
$M / L_{\text {bulge }}$ & Razão massa-luminosidade do bojo \\
$M / L_{\text {disk }}$ & Razão massa-luminosidade do disco \\
$M / L_{\text {bar }}$ & Razão massa-luminosidade da barra \\
$M_{\text {bulge }}$ & Massa do bojo \\
$M_{\text {disk }}$ & Massa do disco \\
$M_{\text {bar }}$ & Massa da barra \\
$z$ & Redshift da galáxia \\
\hline
\end{tabular}




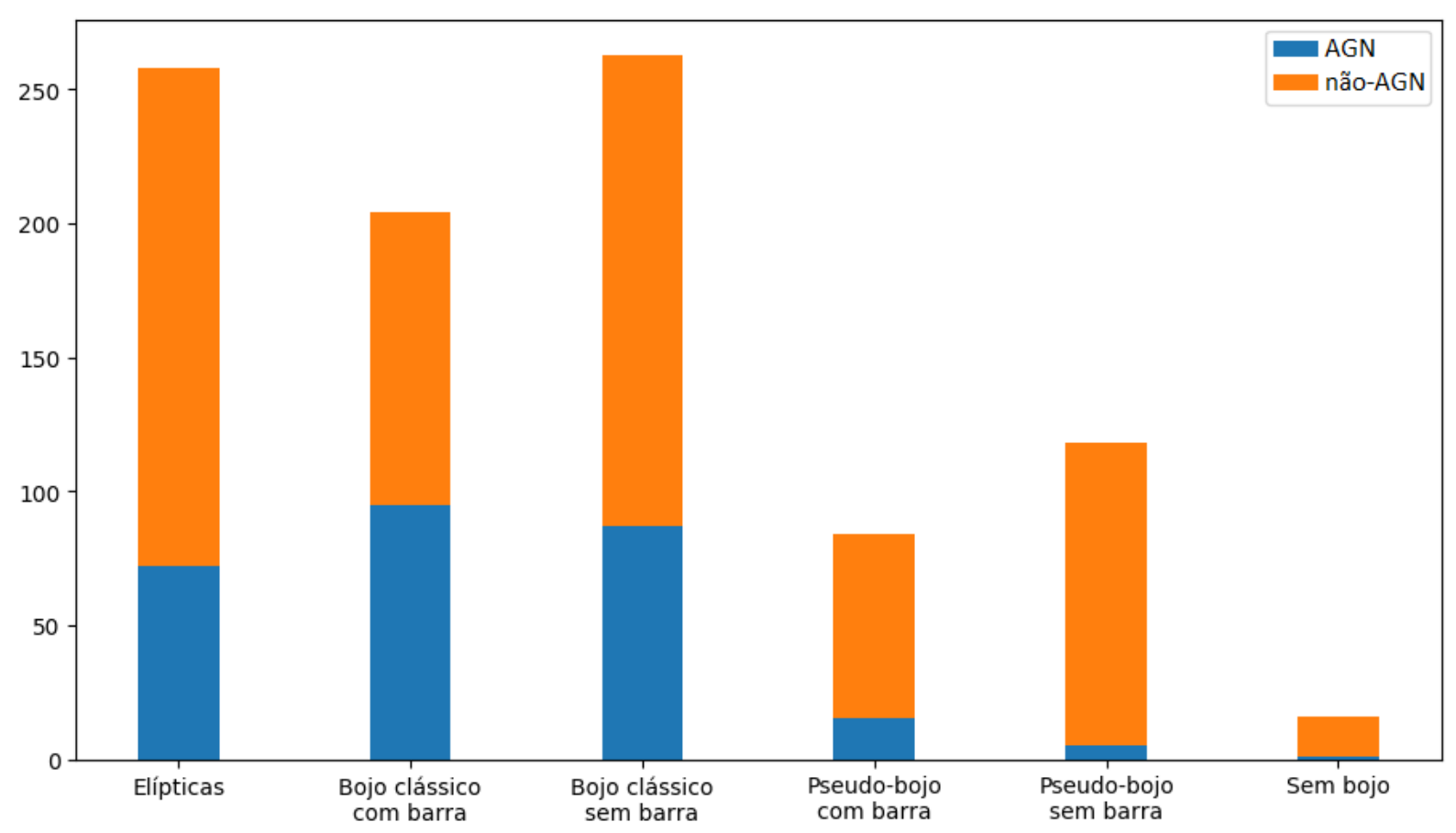

Figura 2.6: Distribuição de morfologias das galáxias da amostra de Gadotti (2009). Barras azuis representam o número de galáxias AGN para cada grupo, enquanto barras laranja representam o número de galáxias não-AGN. 
Capítulo 3

\section{Metodologia}

Este capítulo apresenta a metodologia do nosso trabalho. Aqui apresentamos os modelos de população estelar simples (SSPs) utilizados nos ajustes, assim como o software escolhido. Também discutimos o pré-processamento e correções aplicados aos dados de aglomerados globulares antes dos ajustes de população estelar.

\subsection{Modelos de população estelar simples}

Para este trabalho, adotamos os modelos de Vazdekis et al. (2015), que utilizam as isócronas de BaSTI (Pietrinferni et al. 2004, Pietrinferni et al. 2006), a Função de Massa Inicial (IMF, do inglês Initial Mass Function) bimodal de Vazdekis et al. (1996) e duas bibliotecas de espectros estelares: uma empírica (MILES Sánchez-Blázquez et al. 2006) e uma sintética (Coelho et al. 2005). As SSPs cobrem idades de 0.03 a 14.0 Giga-anos, $[\mathrm{Fe} / \mathrm{H}]$ de -2.27 a 0.4 , abundâncias $\alpha$ com valores solar $([\alpha / \mathrm{Fe}]=0.0)$ e enriquecido $([\alpha / \mathrm{Fe}]$ =0.4), e possuem cobertura espectral de 3540.5-7409.6 $\AA$.

A biblioteca possui mais de mil modelos, porém adotamos apenas 324 deles, escolhendo um a cada três valores de idade disponíveis. Os valores usados foram 0.03, 0.06, 0.09, 0.20, $0.35,0.50,0.80,1.25,2.00,2.75,3.50,4.50,6.00,7.50,9.00,10.50,12.00$ e 13.50 Giga-anos. A figura 3.1 mostra alguns exemplos dos modelos de população estelar simples utilizados.

\subsection{Starlight: Software de ajuste espectral}

Neste trabalho, utilizamos o código Starlight (Cid Fernandes et al., 2005), que é um software de ajuste espectral pixel-a-pixel, ou ajuste espectral por inversão.

Starlight utiliza o algoritmo de Monte Carlo via Cadeias de Markov metropolis (Me- 

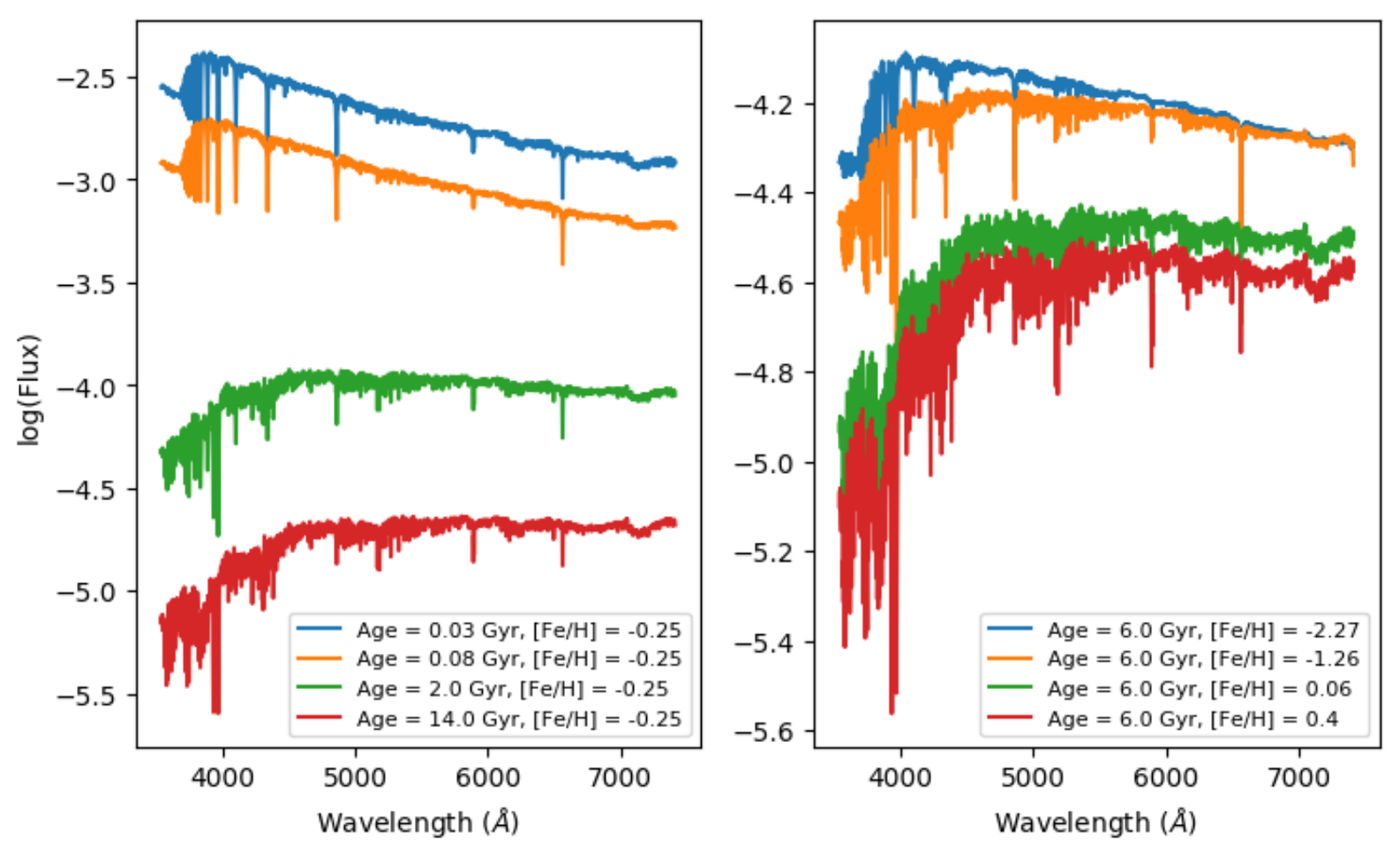

Figura 3.1: Modelos de população estelar simples da biblioteca de Vazdekis et al. (2015). O painel esquerdo mostra quatro modelos de mesma metalicidade e diferentes idades. O painel direito mostra quatro modelos de mesma idade e diferentes metalicidades. Todos os modelos mostrados na imagem possuem $[\alpha / \mathrm{Fe}]$ solar. Os valores de fluxo são dados em $L_{\lambda} / L_{\odot} M_{\odot}^{-1} \stackrel{\circ}{A^{-1}}$. 


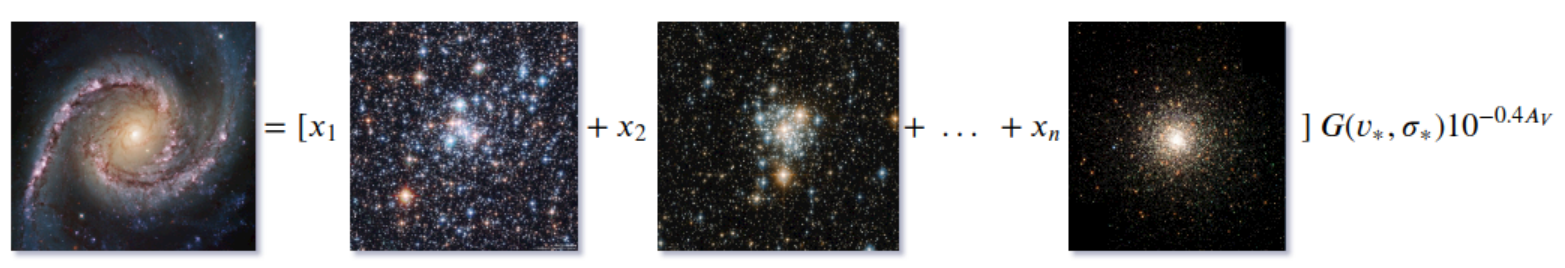

Figura 3.2: Representação visual da equação 3.1. A primeira imagem representa uma galáxia observada, enquanto as demais representam populações estelares simples. Imagens: NASA, ESA, Hubble.

tropolis et al., 1953) combinado com um esquema de simulated annealing para encontrar a melhor combinação de SSPs que represente o espectro observado. O código também ajusta o atenuamento por poeira (na forma de avermelhamento na banda $\mathrm{V}, A_{V}$ ) e os parâmetros cinemáticos (velocidade radial e dispersão de velocidades). O modelo ajustado pelo código é descrito pela equação abaixo:

$$
M_{\lambda}=\sum_{j}^{N_{\star}} x_{j} \operatorname{SSP}_{j, \lambda} \otimes G\left(v_{\star}, \sigma_{\star}\right) 10^{-0.4 A_{V_{j}}\left(q_{\lambda}-q_{\lambda_{0}}\right)}
$$

onde $x_{j}$ é o vetor de população (que corresponde à contribuição de luz de cada $S S P_{j}$ no modelo $M$ ), $G$ é o filtro cinemático (onde $v_{\star}$ é a velocidade radial e $\sigma_{\star}$ é a dispersão de velocidades) e $A_{V}$ é a extinção na banda $V$. Uma representação visual da equação 3.1 é mostrada na Figura 3.2, e a Figura 3.3 ilustra um ajuste espectral feito pelo código.

Utilizando o vetor de população $x_{j}$, é possível inferir parâmetros médios como idades e abundâncias químicas (ponderadas por luz) das populações estelares do objeto estudado, como mostrado nas equações abaixo:

$$
\begin{gathered}
\langle\log (\text { age })\rangle=\sum_{j} x_{j} \cdot \log (\text { age })_{j} \\
\langle[\mathrm{Fe} / \mathrm{H}]\rangle=\sum_{j} x_{j} \cdot[\mathrm{Fe} / \mathrm{H}]_{j} \\
\langle[\alpha / \mathrm{Fe}]\rangle=\sum_{j} x_{j} \cdot[\alpha / \mathrm{Fe}]_{j}
\end{gathered}
$$




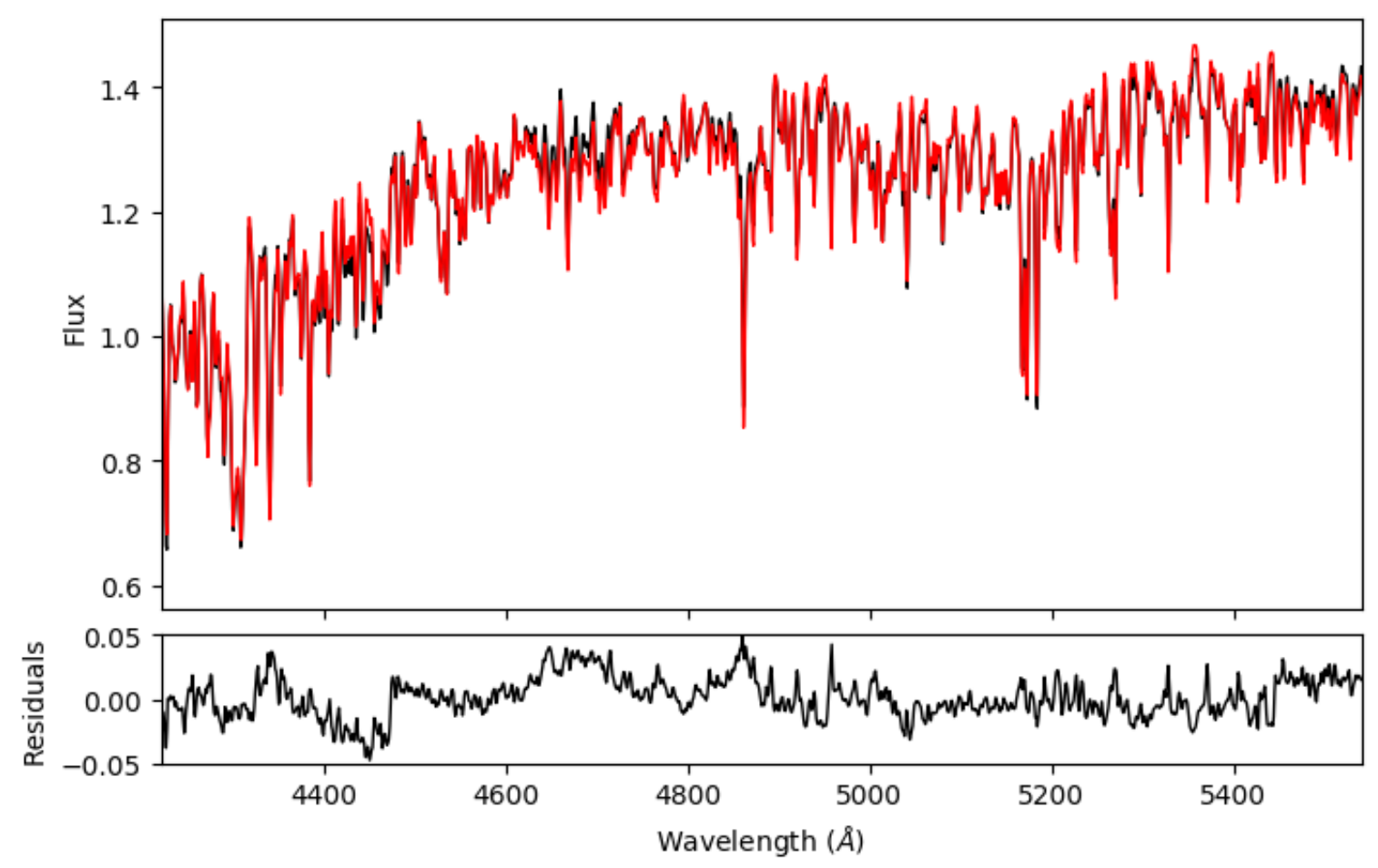

Figura 3.3: Exemplo de ajuste espectral feito pelo Starlight. O painel superior mostra o espectro (fluxo por comprimento de onda) observado no arm B7000 do aglomerado NGC 104 (47 Tuc) da biblioteca WAGGS em preto, e o modelo ajustado pelo STARLIGHT em vermelho. O painel inferior mostra o resíduos do ajuste (fluxo observado menos fluxo sintético) por comprimento de onda. Crédito: Gonçalves et al. (submetido) 
STARLIGHT também retorna o $\chi^{2}$ do ajuste de cada SSP individual aos dados, permitindo ao usuário trabalhar com ajustes de população simples e de multi-populações. Se o vetor de população for substituído por $M_{-} c o r{ }_{j}$ (que é o peso de cada $S S P_{j}$ no modelo, ponderado por massa e corrigido pela perda de massa ocorrida devido aos processos de evolução estelar), obtêm-se os mesmos parametros das equações $3.2,3.4$ ponderados por massa.

\subsection{Pré-processamento dos dados da biblioteca WAGGS}

Antes da análise de populações estelares, fizemos um processamento dos espectros da biblioteca WAGGS de modo a trazê-los para o rest-frame e convoluí-los para a mesma resolução espectral das SSPs. Tal preparação permite que o ajuste espectral com o STARLIGHT seja feito com os parâmetros cinemáticos fixados.

Utilizamos os valores de velocidades radiais medidos pela Profa. Paula Coelho, usando a task fxcor do IRAF'. Cada observação foi correlacionada a três templates, representando estrelas de diferentes tipos espectrais, obtidas da biblioteca de espectros sintéticos de Coelho (2014). Os parâmetros atmosféricos - $\mathrm{T}_{\text {eff }}, \log g,[\mathrm{Fe} / \mathrm{H}]$ e $[\alpha / \mathrm{Fe}]-\operatorname{dos}$ templates adotados foram: $4250,+2.5,-1.0,+0.4 ; 5750,+4.0,-0.5,+0.0$, e; $10000,+2.0,-0.5,+0.0$. Portanto, para cada observação foram medidos três valores de velocidade radial, e adotamos o valor de menor incerteza. As velocidades medidas com os três templates foram consistentes umas com as outras para a maior parte dos dados, e os valores adotados são mostrados na Tabela A.1. Para o 'arm' U7000 de nove aglomerados, os valores divergiram devido ao baixo sinal-ruído. Nesses casos, nenhum valor é mostrado na Tabela A.1 e o valor do 'arm' B7000 foi adotado na correção.

Os dados da biblioteca WAGGS tem resolução maior do que as SSPs utilizadas. Desse modo, fizemos uma degradação dos espectros observados para que não houvesse discrepâncias entre as resoluções dos modelos e dos espectros observados.

A resolução de um espectro é dada por:

$$
R=\frac{\lambda}{\Delta \lambda}=\frac{\lambda}{F W H M}
$$

onde $\lambda$ é o comprimento de onda de um dado pixel e $F W H M$ é o full width at half

\footnotetext{
$1 \longdiv { \text { http://ast.noao.edu/data/software } }$
} 
maximum, ou largura a meia-altura da linha espectral.

O valor de FWHM, por sua vez é dado por:

$$
F W H M \sim 2.355 \sigma
$$

Portanto, podemos usar um filtro gaussiano, dependente apenas de $\sigma$ para diminuir a resolução de um espectro e trazê-lo para um valor de $R$ desejado.

A resolução dos espectros da biblioteca WAGGS não é constante, mas sim dependente de $\lambda$. A Figura 3.4 mostra os ajustes de resolução por comprimento de onda dos espectros observados. Os valores foram medidos ajustando-se gaussianas sobre as linhas de emissão dos espectros de calibração de comprimento de onda, que foram reduzidos da mesma maneira que os espectros dos aglomerados. Nos dados, foram ajustados polinômios quadráticos, que resultaram nas equações 3.7 a 3.10 . Tais medições foram feitas por Christopher Usher durante o processo de redução dos dados.

$$
\begin{gathered}
R_{U 7000} \approx-3.14 \times 10^{-3} \lambda^{2}+2.70 \times 10^{1} \lambda-5.09 \times 10^{4} \\
R_{B 7000} \approx 7.50 \times 10^{-4} \lambda^{2}-5.93 \lambda+1.74 \times 10^{4} \\
R_{R 7000} \approx 1.39 \times 10^{-4} \lambda^{2}-1.94 \times 10^{-1} \lambda+2.75 \times 10^{3} \\
R_{I 7000} \approx-2.04 \times 10^{-7} \lambda^{2}+8.14 \times 10^{-1} \lambda+4.10 \times 10^{2}
\end{gathered}
$$

Sabendo que $\sigma_{S S P}, \sigma_{W A G G S}$ e $\sigma_{\text {convolucão }}$ se relacionam como

$$
\sigma_{S S P}^{2}=\sigma_{W A G G S}^{2}+\sigma_{\text {convolucão }}^{2}
$$

e utilizando as equações 3.5, 3.6 e 3.11, obtemos que o filtro gaussiano que degrada os espectros da biblioteca WAGGS para a resolução das SSPs de Vazdekis et al. (2015) possui $\sigma_{\text {convolucão }}^{A}$ igual a:

$$
\sigma_{\text {convolucão }}^{\stackrel{\circ}{A}}=\frac{\sqrt{\frac{\lambda^{2}}{R_{S S P}^{2}}-\frac{\lambda^{2}}{R_{W A G G S}^{2}}}}{2.355}
$$



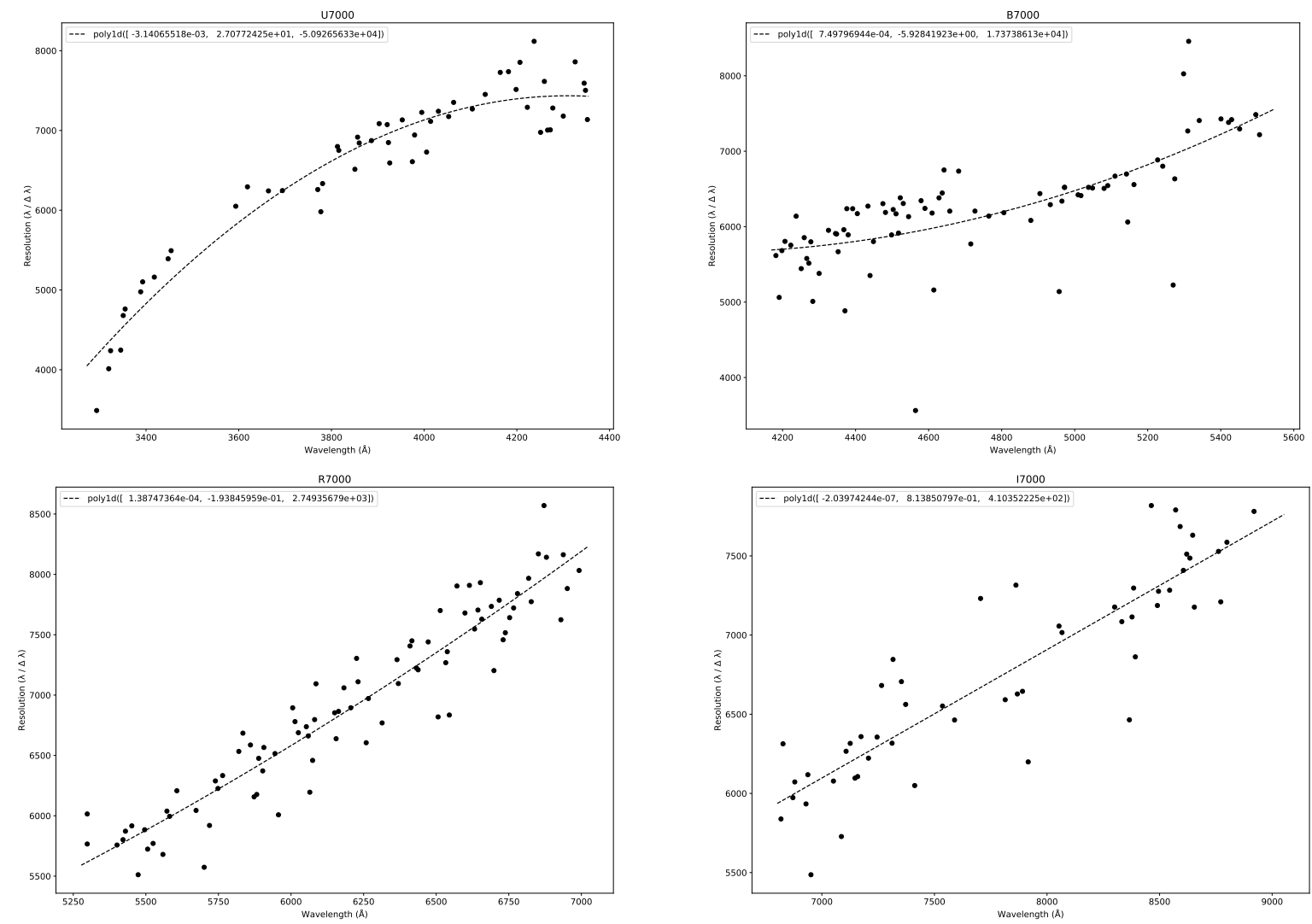

Figura 3.4: Resolução por comprimento de onda para os dados da biblioteca WAGGS (U7000, B7000, R7000 e I7000, respectivamente). Crédito: Christopher Usher (comunicação privada).

Assim, aplicamos a convolução gaussiana pixel-a-pixel usando as funções gaussian e convolve do pacote scipy do PYTHON, que recebe o espectro a ser degradado e um filtro gaussiano dependente apenas de $\sigma$. Como a função convolve faz a convolução em função de pixeis, e não de angstroms, precisamos converter os valores de $\sigma$ para que tenham

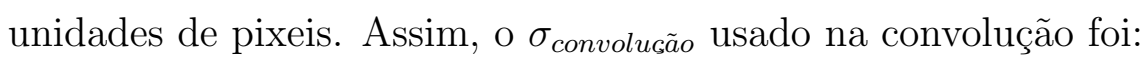

$$
\sigma_{\text {convolucão }}=\frac{\sigma_{\text {convolucão }}^{\stackrel{\circ}{A}}}{\text { pix_bin }}
$$

onde pix_bin é o intervalo entre cada píxel do espectro. 
Capítulo 4

\section{Resultados e análises}

Para a investigar a influência do intervalo de comprimento de onda do ajuste nos resultados, realizamos ajustes de população estelar nos espectros da biblioteca WAGGS. Tais ajustes foram feitos usando cinco diferentes regiões espectrais, mostrados na Tabela 4.1 ,

A Seção 4.1 faz uma análise da qualidade dos ajustes, enquanto as demais discutem os resultados dos parâmetros calculados: $A_{V}$, idades, $[\mathrm{Fe} / \mathrm{H}]$ e anundância de elementos $\alpha$.

Todos os resultados mostrados nesse capítulo são apresentados em um artigo (Gonçalves et al., submetido a MNRAS).

\subsection{Qualidade dos ajustes}

A medida de qualidade dos ajustes feitos pelo StARLIGHT em seus arquivos de saída é dada em termos do $\chi^{2}$ e da diferença média relativa entre modelo e observação adev, ou $\Delta_{\lambda}:$

$$
\Delta_{\lambda}=\frac{1}{N} \sum_{\lambda}\left|\frac{f_{\text {model }}(\lambda)-f_{\text {obs }}(\lambda)}{f_{\text {obs }(\lambda)}}\right| \sim \frac{1}{S N R}
$$

onde $N$ é o número de pixels, $f_{\text {model }}$ é o fluxo ajustado e $f_{\text {obs }}$ é o fluxo observado.

Na Figura 4.1 são ilustradas as distribuições de $\chi^{2}$ e $\Delta_{\lambda}$ para todos os ajustes. Apenas os valores de $\Delta_{\lambda}$ até $5 \%$ são mostrados, apesar de eles poderem atingir valores muito mais altos para alguns poucos ajustes. Por exemplo, NGC2004, cujos espectros possuem baixíssimo sinal-ruído (SNR 11.0 em B7000 e SNR 1.0 em U7000 e R7000), resulta em ajustes com $\Delta_{\lambda} \sim 18 \%$ em R7000 e $\Delta_{\lambda} \sim 500 \%$ em U7000. Todos esses casos estão listados na legenda da Figura 4.1. 
Tabela 4.1 - Intervalos de cobertura espectral utilizados neste trabalho.

\begin{tabular}{|c|c|c|c|c|}
\hline \multirow[t]{2}{*}{ Nome } & \multirow[t]{2}{*}{ Intervalo $(\AA)$} & \multirow[t]{2}{*}{ Notas } & \multicolumn{2}{|c|}{ Intervalo de normalização $(\AA)$} \\
\hline & & & Para a base & Para os observados \\
\hline$R_{U}$ & $3540.5-4350.0$ & Intersecção entre U7000 e a cobertura das SSPs. & 4250.0 & $4240.0-4260.0$ \\
\hline$R_{B}$ & $4170.0-5540.0$ & Cobertura de B7000. & 4250.0 & $4240.0-4260.0$ \\
\hline$R_{R}$ & $5280.0-7020.0$ & Cobertura de R7000. & 7000.0 & $6990.0-7010.0$ \\
\hline$R_{W}$ & $4828.0-5364.0$ & Intervalo favorecido por Walcher et al. (2009). & 5050.0 & $5040.0-5060.0$ \\
\hline$R_{M}$ & $3540.5-7409.6$ & Cobertura das SSPs. & 4250.0 & $4240.0-4260.0$ \\
\hline
\end{tabular}
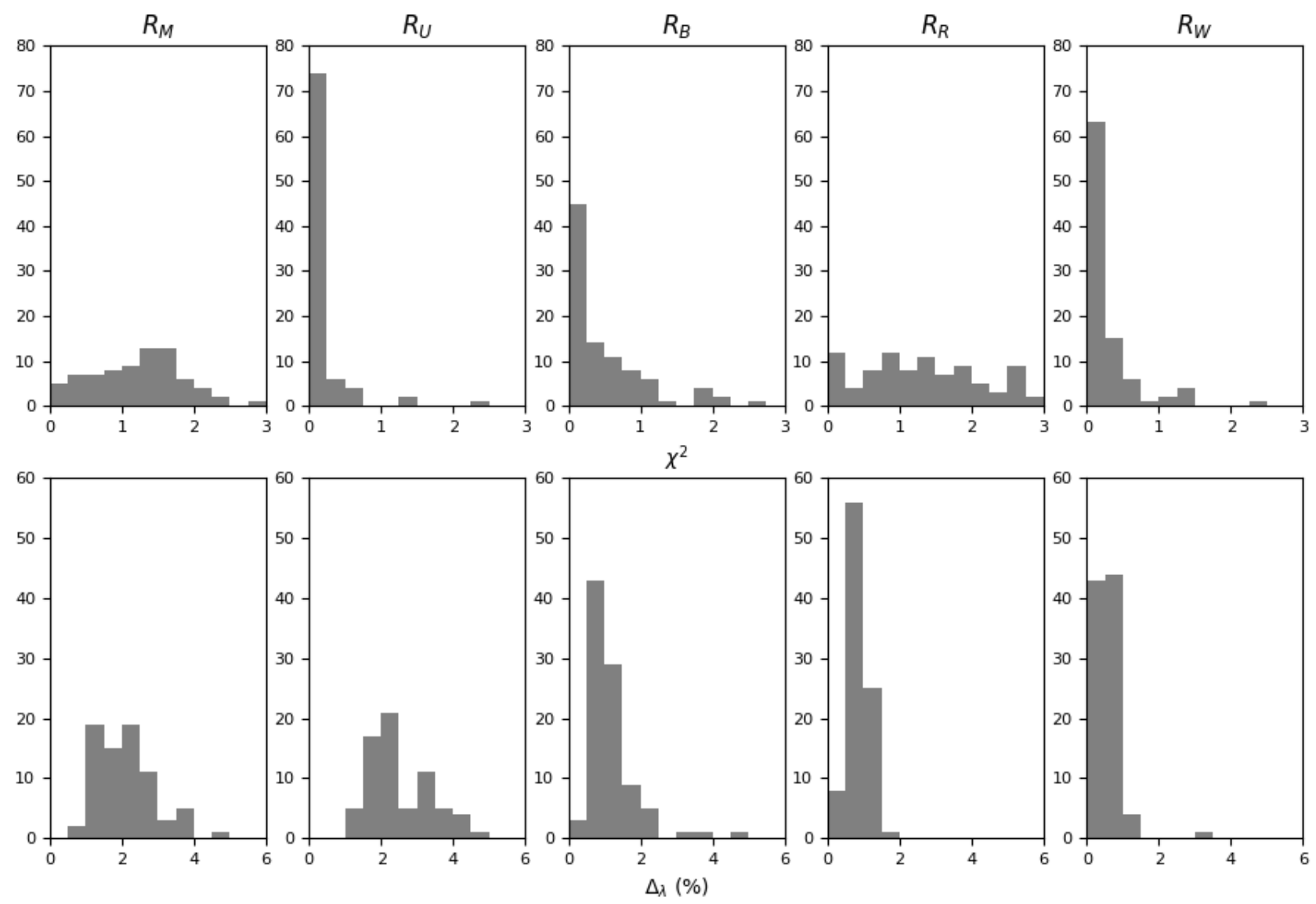

Figura 4.1: Distribuições de $\chi^{2}$ (primeira linha) e $\Delta_{\lambda}$ (segunda) para os ajustes feitos com Starlight dos espectros da biblioteca WAGGS. Cada coluna apresenta tais distribuições para um intervalo de comprimento de onda diferente (Tabela 4.1). Para $\Delta_{\lambda}$, são mostrados apenas valores menores que $5 \%$. Para os seguintes casos, $\Delta_{\lambda}$ não é mostrado na segunda linha da figura: NGC1846- $R_{M}\left(\Delta_{\lambda} \sim 28 \%\right)$, NGC6440$R_{M}\left(\Delta_{\lambda} \sim 31 \%\right)$, NGC6553- $R_{M}\left(\Delta_{\lambda} \sim 24 \%\right)$, NGC6528- $R_{M}\left(\Delta_{\lambda} \sim 32 \%\right)$, NGC2004- $R_{M}\left(\Delta_{\lambda} \sim 58 \%\right)$, NGC2004- $R_{R}\left(\Delta_{\lambda} \sim 18 \%\right)$, NGC2004- $R_{U}\left(\Delta_{\lambda} \sim 492 \%\right)$, NGC2009- $R_{U}\left(\Delta_{\lambda} \sim 21 \%\right)$, NGC6553- $R_{U}$ $\left(\Delta_{\lambda} \sim 51 \%\right)$, NGC6440- $R_{U}\left(\Delta_{\lambda} \sim 55 \%\right)$, NGC1846- $R_{U}\left(\Delta_{\lambda} \sim 98 \%\right)$ e NGC6528- $R_{U}\left(\Delta_{\lambda} \sim 115 \%\right)$. Crédito: Gonçalves et al. (submetido) 
Outro aspecto relacionado à qualidade dos ajustes foi discutido em Cid Fernandes (2018), onde o autor apresenta um teste simples que torna possível verificar se houve ou não convergência da Cadeia de Markov durante o ajuste. O output do código mostra, além do $\chi^{2}$ geral do ajuste de multi-populações, um $\chi_{S S P_{j}}^{2}$ de cada $S S P_{j}$ individual ajustada sobre os dados. De acordo com o autor, um bom ajuste resulta em um $\chi^{2}$ menor que qualquer um dos $\chi_{S S P_{j}}^{2}$. Caso contrário, o ajuste não é confiável.

No caso descrito neste trabalho, os ajustes de apenas quatro espectros (Fornax3- $R_{U}$, Fornax5- $R_{R}$, NGC2004- $R_{U}$ e NGC7099- $R_{B}$ ) não cumprem tal requisito, e seus resultados (assim como todos aqueles em que $\Delta_{\lambda}>5 \%$ ) foram removidos das análises posteriores.

\subsection{Avermelhamento}

Apesar de a informação sobre os parâmetros astrofísicos estar presente em todos os pixels do espectro, diferentes parâmetros causam impactos distintos na distribuição de energia observada. Avermelhamento e idades por exemplo, causam um impacto maior no formato do contínuo espectral, enquanto que abundâncias químicas tendem a causar maiores modificações nas linhas de absorção espectrais.

A Figura 4.2 mostra os valores de $A_{V}$ obtidos pelo Starlight para os espectros da biblioteca WAGGS (exceto os excluídos da análise discutidos na seção anterior) em cada um dos intervalos de comprimento de onda estudados (Tabela 4.1) em comparação com valores obtidos da literatura (Harris 1996, McLaughlin e van der Marel 2005b). Para a maioria dos ajustes, os valores resultantes são próximos dos valores de referência. A dispersão entre os valores calculados e de referência é de 0.10 para $R_{M}, 0.25$ para $R_{U}, 0.12$ para $R_{B}, 0.31$ para $R_{R}$ e 0.14 para $R_{W}$.

Nos ajustes, permitimos que a janela de possíveis valores de $A_{V}$ começasse em -1. Gallazzi et al. (2005) e Mateus et al. (2006) discutem algumas razões pelas quais a possibilidade de ajustes com valores de $A_{V}$ negativos deve ser considerada, majoritariamente relacionadas à inconsistências nas bases de modelos de SSP. Claramente, nestes casos específicos, o valor resultante para $A_{V}$ não possui significado físico e passa a ser apenas um meio utilizado pelo algoritmo para diminuir o $\chi^{2}$ do ajuste. Mas vale ressaltar que a maioria dos casos onde $A_{V_{\text {Starlight }}}<0$ ocorre quando os dados de U7000 são utilizados, que correspondem aos espectros com valores de sinal-ruído muito baixos (ver Figura 2.3). 


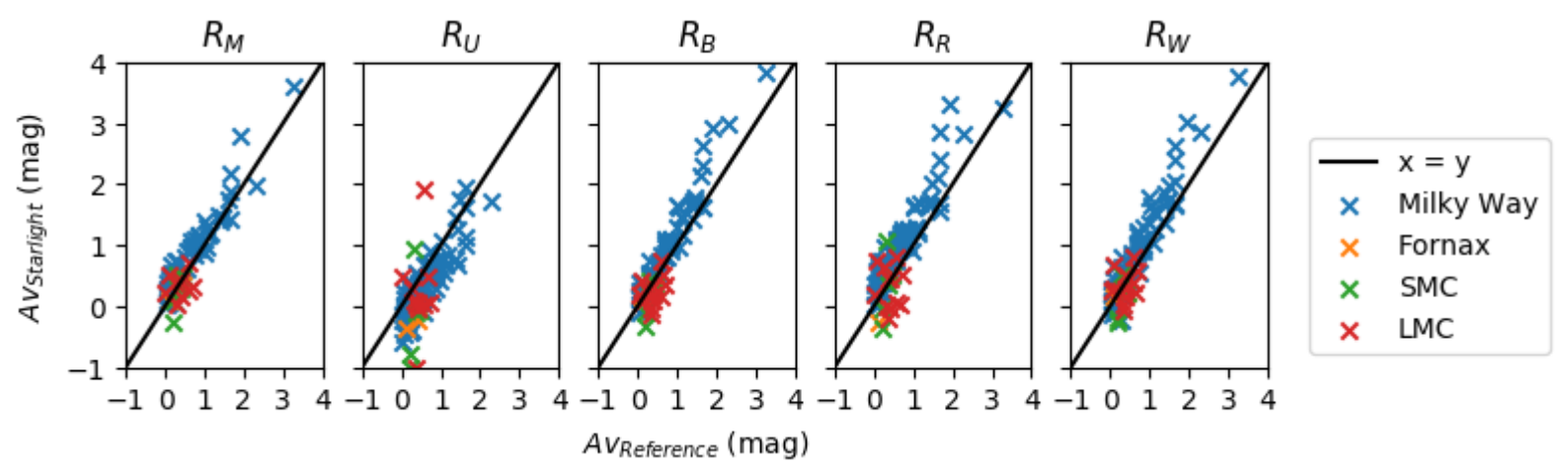

Figura 4.2: Valores de $A_{V}$ calculados pelo StaRLight comparados com valores de referência. Diferentes paineis mostram resultados dos ajustes nos diferentes intervalores de comprimento de onda analizados neste trabalho. A linha $\mathrm{x}=\mathrm{y}$ é mostrada em preto. As cores indicam as galáxias hospedeiras dos aglomerados. Crédito: Gonçalves et al. (submetido)

\subsection{Idades}

Como referência, adotamos valores de idade provenientes de ajustes de isócronas a diagramas cor-magnitude em trabalhos da literatura (ver Tabela 2.2). Mas quando trabalhamos com idades inferidas a partir de espectros de luz integrada, várias definições do que é uma "idade"podem ser empregadas. Portanto, abaixo apresentamos as diferentes definições utilizadas neste trabalho:

- Idade de isócrona $t_{i s o}$ : Idade inferida a partir de ajustes de isócronas, usando CMDs e modelos de evolução estelar.

- Idade média ponderada por luz $t_{\text {light }}$ : Idade média levando em conta o ajuste de multi-populações, onde a soma é ponderada pela contribuição de luz $x_{j}$ de cada $S S P_{j}$ no ajuste feito pelo Starlight. Esta é a definição mostrada na Equação 3.2 .

- Idade média ponderada por massa $t_{m a s s}$ : Idade média levando em conta o ajuste de multi-populações, onde a soma é ponderada pela contribuição de massa de cada $S S P_{j}$ no ajuste feito pelo Starlight.

- Idade de SSP $t_{S S P}$ : Idade do modelo de população estelar simples (sem mistura de populações) que melhor se ajusta ao observado.

- Idade da maior contribuição na mistura de populações $t_{\max x_{j}}$ : Tomando a mistura de populações, $t_{\max x_{j}}$ é a idade da SSP que contribui com a maior porcen- 
tagem de luz ao modelo ajustado.

As quatro definições acima também se aplicam para outros parâmetros, como $[\mathrm{Fe} / \mathrm{H}]$ e $[\alpha / \mathrm{Fe}]$.

A Figura 4.3 mostra os valores resultantes dos ajustes feitos pelo STARLIGHT comparados com os valores de idades de isócrona, para todas as definições listadas acima. Para grande parte dos objetos jovens e de idade intermediária, os valores inferidos se aproximam dos valores de isócronas em $R_{M}, R_{U}, R_{B}$ e $R_{W}$, independente da definição de idade adotada. Os valores obtidos com os espectros de R7000 mostram uma dispersão maior, o que é esperado, dado que as linhas espectrais que mais influenciam no cálculo das idades concentram-se na parte mais azul do espectro.

No caso de sistemas mais antigos $\left(\log \left(t_{i s o}\right)>10\right)$, há uma maior discrepância entre os valores calculados e os valores de referência. Há ainda uma forte dependência entre os erros nas idades com a metalicidade, estando os objetos mais pobres em metais mais distantes da linha $x=y$. Esse efeito pode ser visto de modo mais claro na Figura 4.4, que mostra a comparação dos valores obtidos com os de referência em escala linear, e na Figura 4.5 , que mostra a dependência de $\Delta t_{i}=t_{i}-t_{\text {iso }} \operatorname{com}[\mathrm{Fe} / \mathrm{H}]$ (sendo $t_{i}$ qualquer das diferentes idades definidas no início desta seção).

Uma das hipóteses para explicar tais resultados é o excesso de luz azul observado em alguns aglomerados globulares velhos e pobres em metais, em comparação com modelos de SSP de mesma idade e metalicidade. Um exemplo pode ser visto na Figura 4.6. A interpretação clássica para tal efeito é a presença de um ramo horizontal extendido ou de blue-stragglers. Tais estrelas geralmente não são levadas em conta na modelagem das SSPs, logo, o algoritmo pode interpretar tal "excesso" de luz azul como sendo proveniente de populações estelares jovens. Desse modo, a idade calculada acaba sendo menor que a idade proveniente de ajuste de isócronas.

Em Fernandes e Delgado (2010), os autores afirmam que a falta de estrelas velhas e azuis nos modelos de SSP pode ser o motivo pelo qual obtemos valores de $\chi^{2}$ menores em ajustes de multi-populações em aglomerados estelares quando, devido à natureza desses objetos, deveríamos ser capazes de conseguir melhores resultados utilizando ajustes de população simples.

No contexto de ajustes espectrais, Koleva et al. (2008) abordaram tal problema uti- 

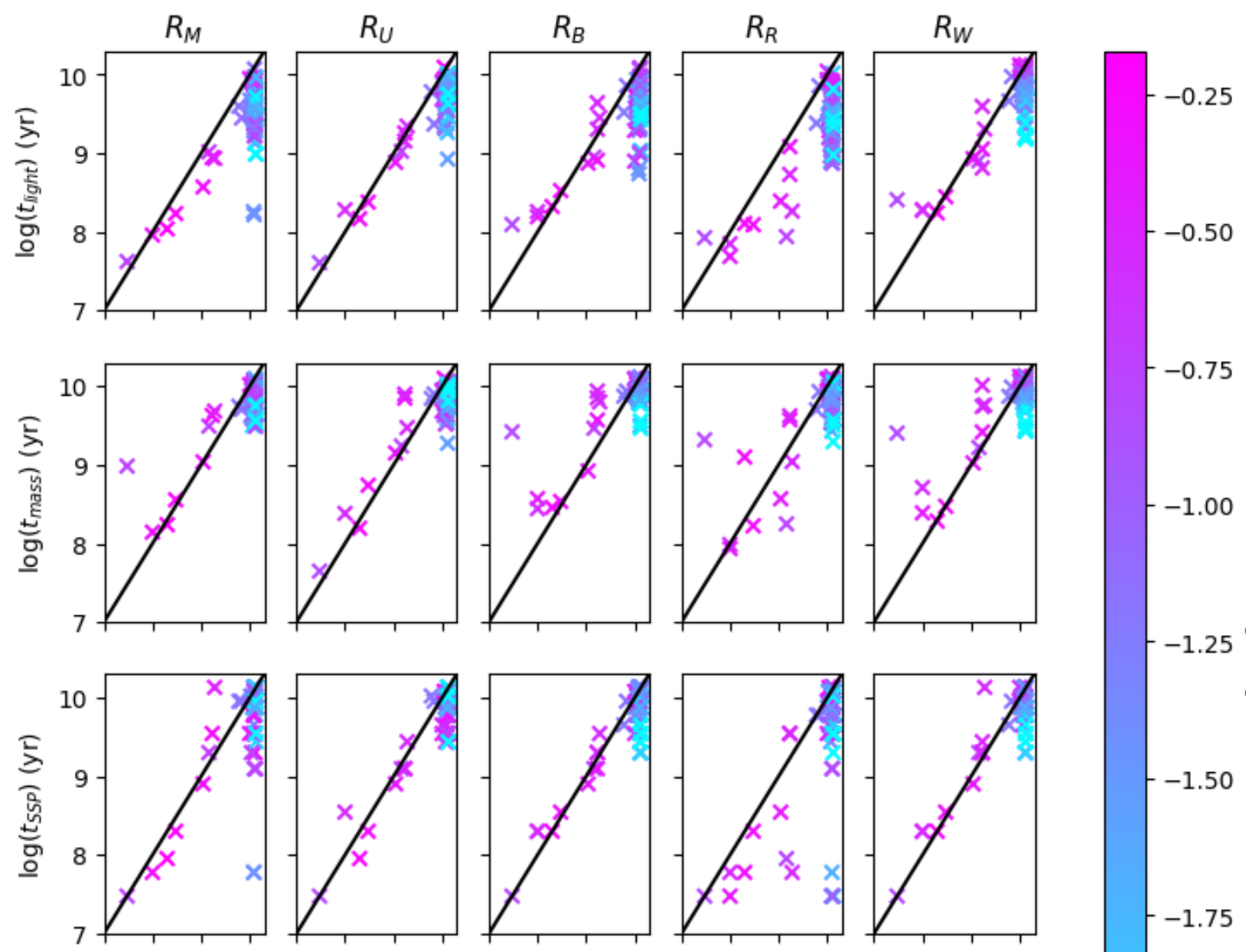

$-1.25 \underset{\text { 写 }}{\text { 金 }}$
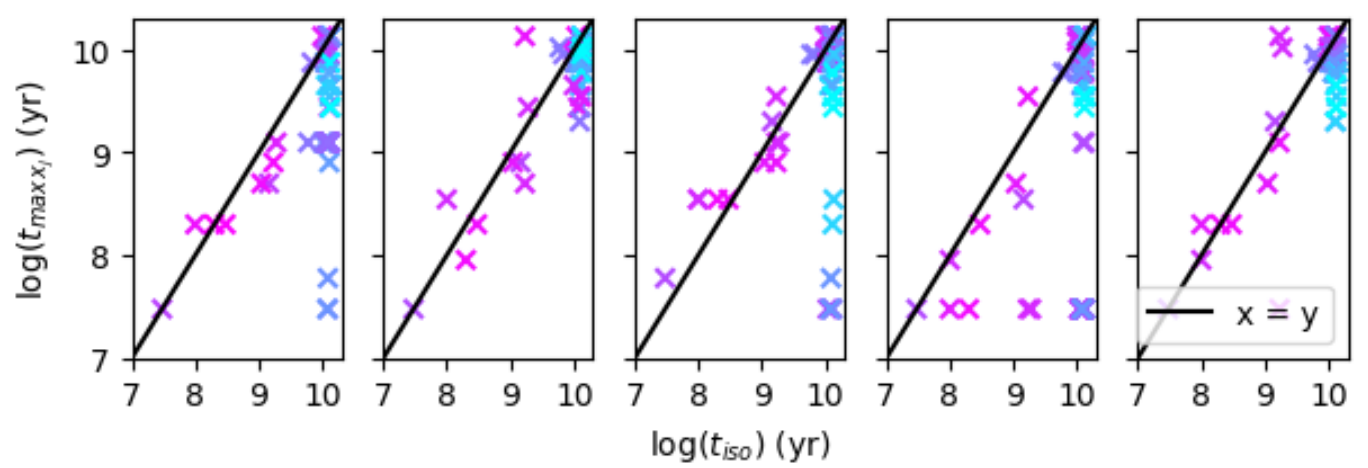

$-1.75$

$-1.50$

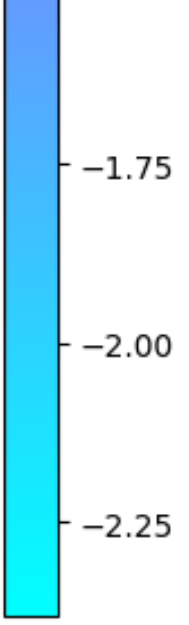

Figura 4.3: Idades resultantes dos ajustes espectrais (eixo y) versus idades provenientes de ajustes de isócrona (eixo x), em escala logarítimica. Cada linha mostra os resultados para uma das diferentes definições de idade descritas na Seção 4.3 . Cada coluna representa um dos intervalos de comprimento de onda testados neste trabalho (Tabela 4.1). A linha $\mathrm{x}=\mathrm{y}$ é mostrada em preto. Marcadores são coloridos por $[\mathrm{Fe} / \mathrm{H}]_{r e f}$. Crédito: Gonçalves et al. (submetido) 


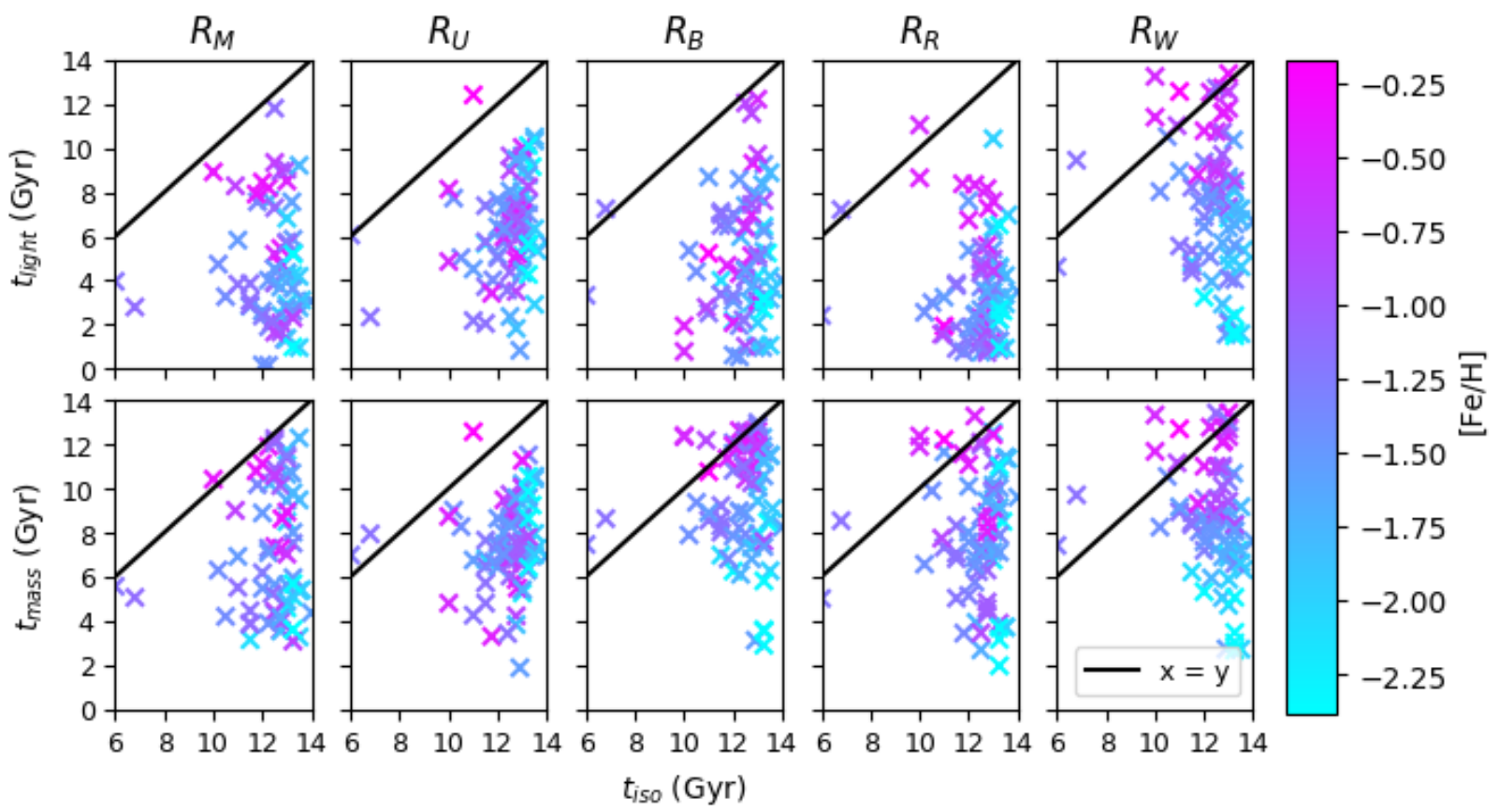

Figura 4.4: Idades resultantes dos ajustes espectrais (eixo y) versus idades provenientes de ajustes de isócrona (eixo x), em escala linear. A linha superior mostra resultados para idades ponderadas por luz, e a inferior, para idades ponderadas por massa. Cada coluna representa um dos intervalos de comprimento de onda testados neste trabalho (Tabela 4.1). A linha $\mathrm{x}=\mathrm{y}$ é mostrada em preto. Marcadores são coloridos por $[\mathrm{Fe} / \mathrm{H}]_{\text {ref }}$. Crédito: Gonçalves et al. (submetido)
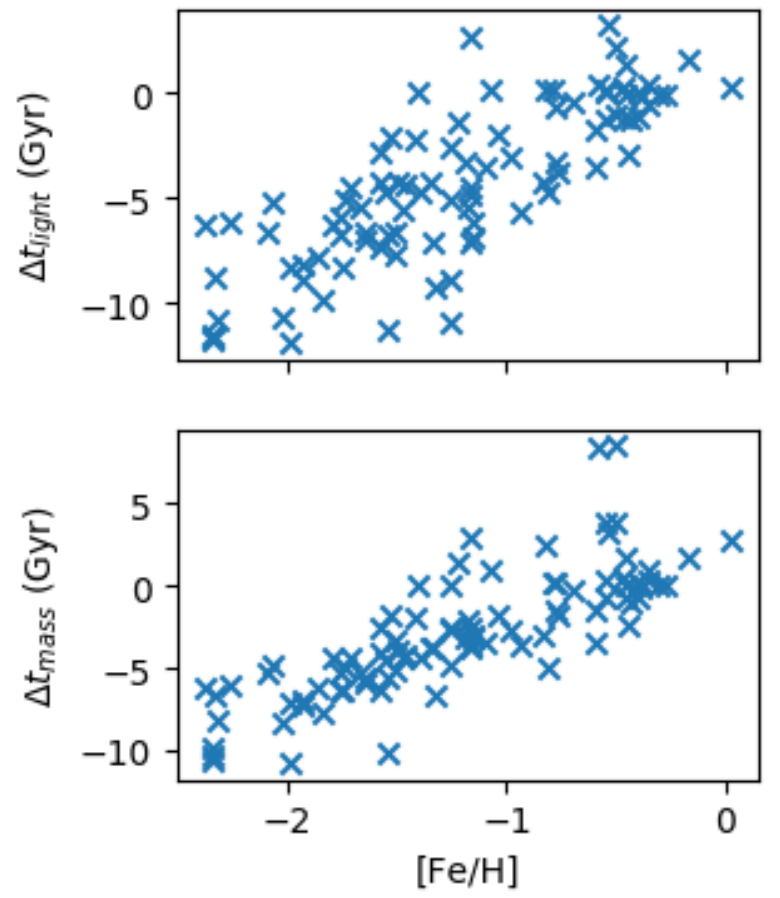

Figura 4.5: Valores de $\Delta t=t_{\text {Starlight }}-t_{\text {iso }}$ (ponderados por luz no painel superior e por massa no painel inferior) versus $[\mathrm{Fe} / \mathrm{H}]$, resultantes do ajuste utilizando o intervalo $R_{W}$. É notável que há uma clara correlação entre a diferença de idade (idade ajustada pelo STARLIGHT menos idade resultante de ajuste de isócrona) e a metalicidade. Crédito: Gonçalves et al. (submetido) 


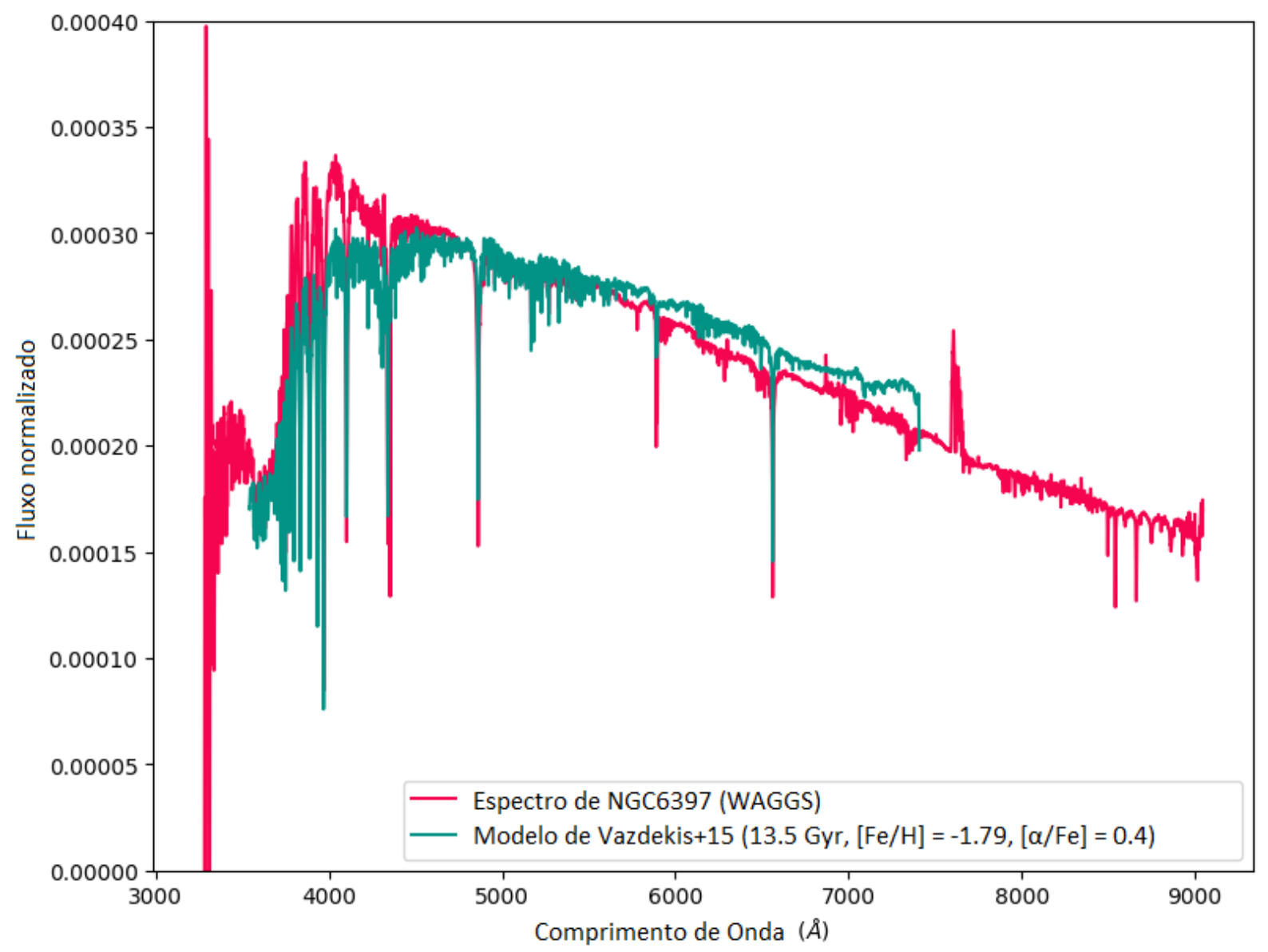

Figura 4.6: Espectro de NGC6397 (em rosa) e o modelo de SSP de Vazdekis et al. (2015) que mais se aproxima dos valores de referência (idade proveniente de ajuste de isócronas e metalicidade proveniente de análise de espectroscopia de alta resolução) para esse aglomerado (em verde). Nota-se o "excesso"de fluxo em comprimentos de onda mais curtos no espectro observado em comparação com o modelo. 
lizando espectros de estrelas quentes juntamente com os modelos de população estelar simples de modo a simular o efeito de um possível ramo horizontal extendido. Assim, eles foram capazes de inferir idades muito mais próximas das idades de isócronas dos aglomerados.

Ocvirk (2010) propõe, ainda, que qualquer resultado que indique um burst de formação estelar em meio a uma população velha com $[\mathrm{Fe} / \mathrm{H}]=[-2,-1.2]$ pode ser interpretada como um artefato caso represente menos de $12 \%$ da luz na região do óptico. Porém, os ajustes aqui estudados nem sempre se aplicam a esse caso, como exemplificam as Figuras 4.7 e 4.8. Elas mostram os resultados dos ajustes em $R_{B}$ para os aglomerados NGC6397 $\left(t_{i s o}=13.5 \mathrm{Gyr},[\mathrm{Fe} / \mathrm{H}]=-2.0\right)$ e NGC1916 $\left(t_{i s o}=12.9 \mathrm{Gyr},[\mathrm{Fe} / \mathrm{H}]=-1.5\right)$, ambos ajustados pelo Starlight com idades muito abaixo de suas respectivas idades resultantes de ajustes de isócronas. Em ambas as figuras, os painéis da esquerda mostram curvas de nível indicando as contribuições de luz $x_{j}$ do melhor ajuste no espaço de parâmetros idade versus metalicidade, enquanto os painéis direitos mostram a distribuição do vetor de população $x_{j}$ dos ajustes para idade. É notável que, em ambos, $\sum\left(x_{j}<1.0\right.$ Gyr $)$ (população jovem) ultrapassa o limite de $12 \%$ definido em Ocvirk (2010). Portanto, acreditamos haver algo mais fundamental na discrepância entre os parâmetros resultantes dos ajustes e os valores de referência para aglomerados pobres em metais.

\subsection{Abundâncias químicas}

Para o parâmetro indicador de metalicidade, $[\mathrm{Fe} / \mathrm{H}]$, utilizamos as mesmas definições descritas na Seção 4.3 .

A Figura 4.9 mostra os valores provenientes dos ajustes feitos com o STARLIGHT comparados com os valores de referência obtidos da literatura. É notável que, apesar de uma diferença sistemática ( $\sim 0.2$ dex), os valores ajustados das metalicidades ponderadas por luz e por massa mostram uma grande concordância com os valores de referência. $[\mathrm{Fe} / \mathrm{H}]_{\mathrm{SSP}}$ e $[\mathrm{Fe} / \mathrm{H}]_{\max x_{j}}$ também mostram alguma correlação, apesar de possuírem um espalhamento mais significativo (chegando em 2.0 dex em alguns casos).

Para o caso de $[\mathrm{Fe} / \mathrm{H}]_{\mathrm{SSP}}$, tal espalhamento pode ser explicado pelo caráter discretizado da base, que possui modelos com $[\mathrm{Fe} / \mathrm{H}]$ igual a $-2.27,-1.79,-1.49,-1.26,-0.96,-0.66,-0.35$, 0.06, 0.26 e 0.40. Possuindo apenas tais valores disponíveis para metalicidade, e não um 
NGC6397_B7000
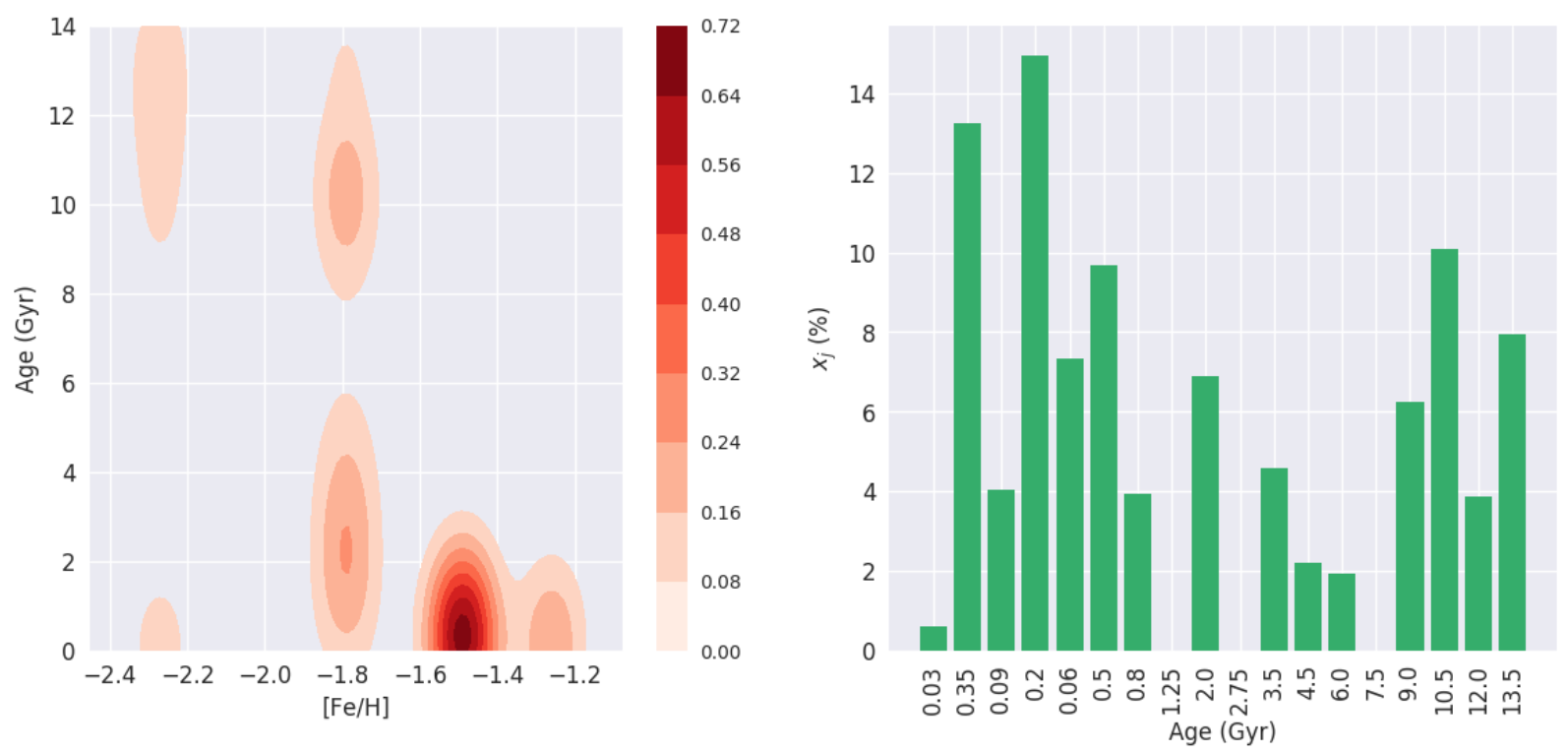

Figura 4.7: Representação visual do resultado do melhor ajuste em $R_{B}$ para o espectro de NGC6397 da biblioteca WAGGS. Esquerda: curvas de nível mostrando a distribuição das SSPs que compõem o melhor ajuste, no espaço de parâmetros idade versus $[\mathrm{Fe} / \mathrm{H}]$. Direita: distribuição dos componentes do vetor de população $x_{j}$ no espaço das idades.

NGC1916_B7000
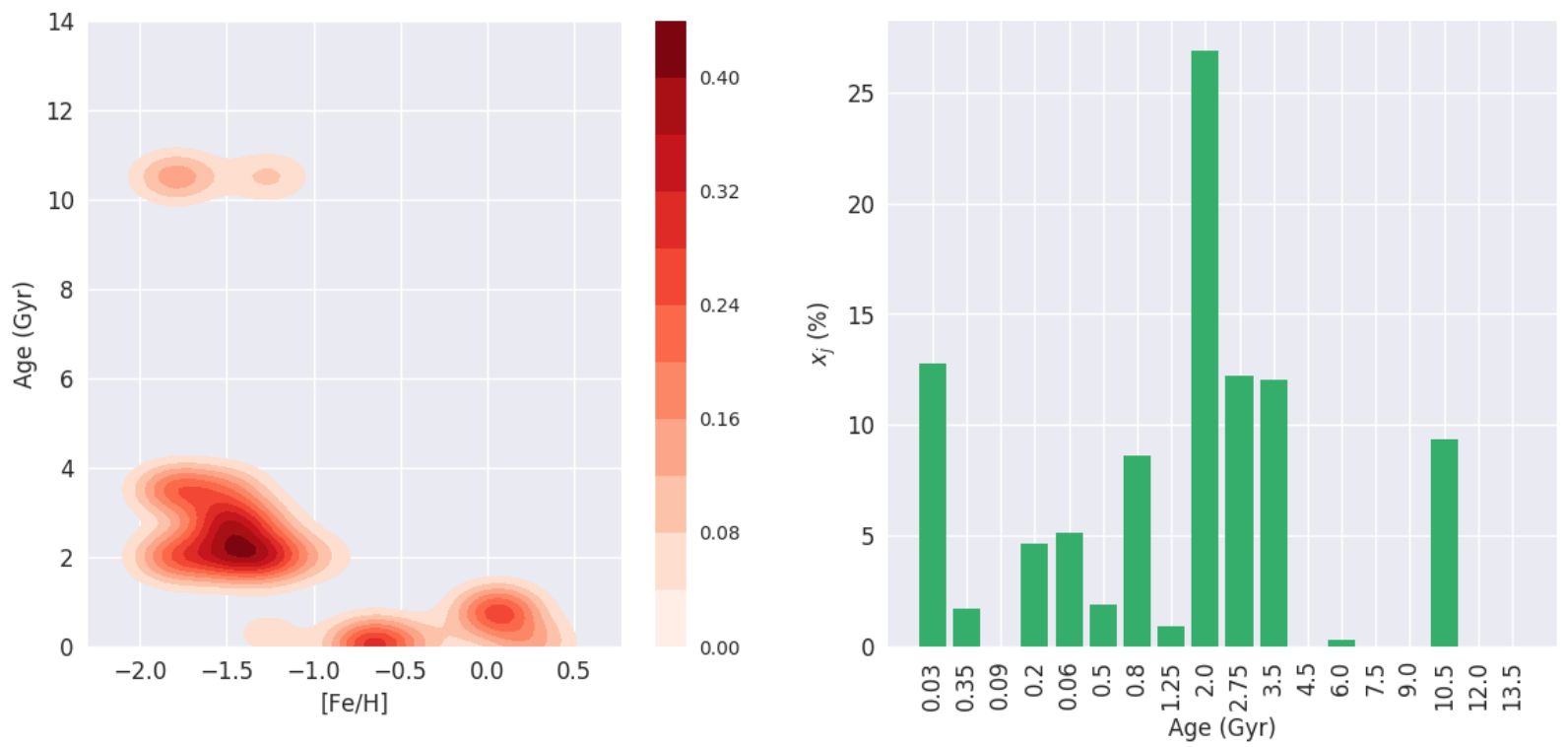

Figura 4.8: Representação visual do resultado do melhor ajuste em $R_{B}$ para o espectro de NGC1916 da biblioteca WAGGS. Esquerda: curvas de nível mostrando a distribuição das SSPs que compõem o melhor ajuste, no espaço de parâmetros idade versus $[\mathrm{Fe} / \mathrm{H}]$. Direita: distribuição dos componentes do vetor de população $x_{j}$ no espaço das idades. 
contínuo de valores como no caso dos ajustes de multi-população, é natural que o algoritmo não seja capaz de fazer um ajuste mais fino e que isso gere um espalhamento maior.

Para $[\mathrm{Fe} / \mathrm{H}]_{\max x_{j}}$, além da discretização da base, também há o fato de que tal parâmetro é, na verdade, uma fração de um outro parâmetro. Tomamos a informação integrada e tentamos ajustar apenas uma parte dela, o que pode ser uma fonte maior de erros. Se, por exemplo, um espectro é ajustado como sendo composto $30 \%$ por uma SSP rica em metais e mais sete SSPs pobres em metais, sendo $10 \%$ cada, o valor final de $[\mathrm{Fe} / \mathrm{H}]_{\max x_{j}}$ indicaria que o objeto é rico em metais.

O parâmetro $[\alpha / \mathrm{Fe}]$, indicador de abundância de elementos- $\alpha$, é de extrema importância em estudos de população estelar, pois funciona como um relógio cósmico da história de formação estelar de uma galáxia (e.g. Matteucci, 2003). Há mais de uma década, tal parâmetro tem sido medido principalmente usando índices espectrais (e.g. Thomas et al., 2005), mas trabalhos como Walcher et al. (2009) e Conroy et al. (2018) mediram esse parâmetro em espectros integrados utilizando ajuste espectral.

A Figura 4.10 apresenta os resultados dos ajustes feitos com o Starlight para $[\alpha / \mathrm{Fe}]$ ponderado tanto por luz quanto por massa. Optamos por mostrar os resultados na forma de distribuições de $\Delta[\alpha / \mathrm{Fe}]=[\alpha / \mathrm{Fe}]_{\text {Starlight }}-[\alpha / \mathrm{Fe}]_{\text {Reference }}$, e nota-se que para quase todos os intervalos de comprimento de onda ajustados (com exceção apenas de $R_{R}$ ), as distribuições mostram picos em torno de zero. Optamos por apresentar os resultados nesse formato devido ao caráter elusivo que os valores de $[\alpha / \mathrm{Fe}]$ podem mostrar em scatter plots, devido ao pequeno intervalo de valores disponíveis - de -0.2 a 0.5 para as observações, e apenas dois valores (0.0 e 0.4$)$ nos modelos.

\subsection{Incertezas}

Até o momento, todos os resultados foram apresentados sem incertezas. Neste tipo de estudo, há diferentes possíveis fontes de erro: eles podem ser de origem observacional, podem ter origem no algoritmo utilizado no ajuste ou ainda vir das incertezas dos modelos de SSP utilizados.

Avaliamos aqui as incertezas de origem observacional de duas maneiras diferentes, discutidas nas subseções a seguir. Uma discussão detalhada a respeito de possíveis erros provenientes dos modelos é apresentada em Coelho et al. (2009), e em Delgado e Fernan- 

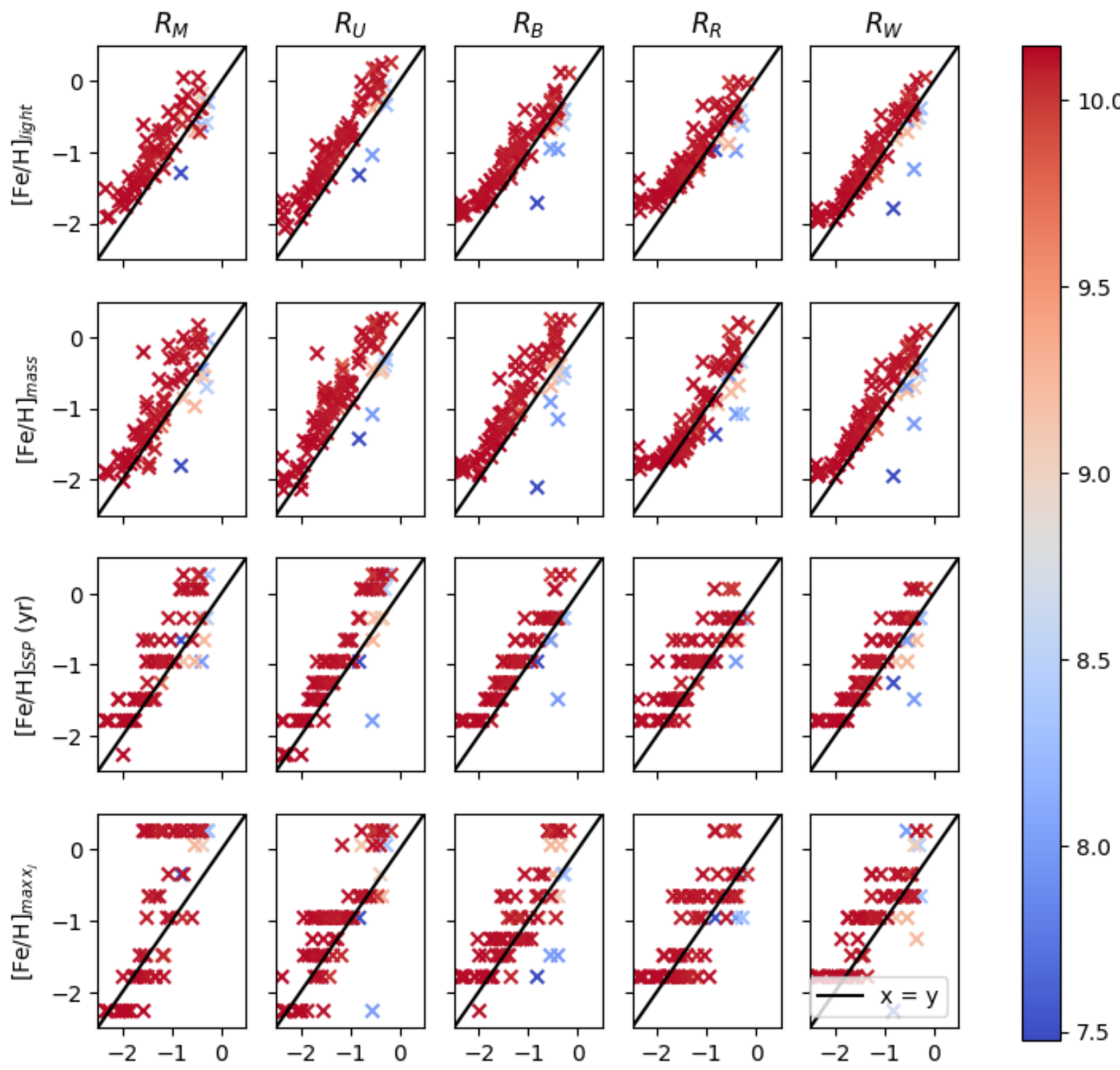

$[\mathrm{Fe} / \mathrm{H}]_{\text {Reference }}$

Figura 4.9: Valores de $[\mathrm{Fe} / \mathrm{H}]$ resultantes dos ajustes espectrais (eixo y) versus valores de referencia (eixo x). Cada linha mostra os resultados para uma das diferentes definições descritas na Seção 4.3. Cada coluna representa um dos intervalos de comprimento de onda testados neste trabalho (Tabela 4.1). A linha $\mathrm{x}=\mathrm{y}$ é mostrada em preto. Marcadores são coloridos por idades de isócrona. Crédito: Gonçalves et al. (submetido) 

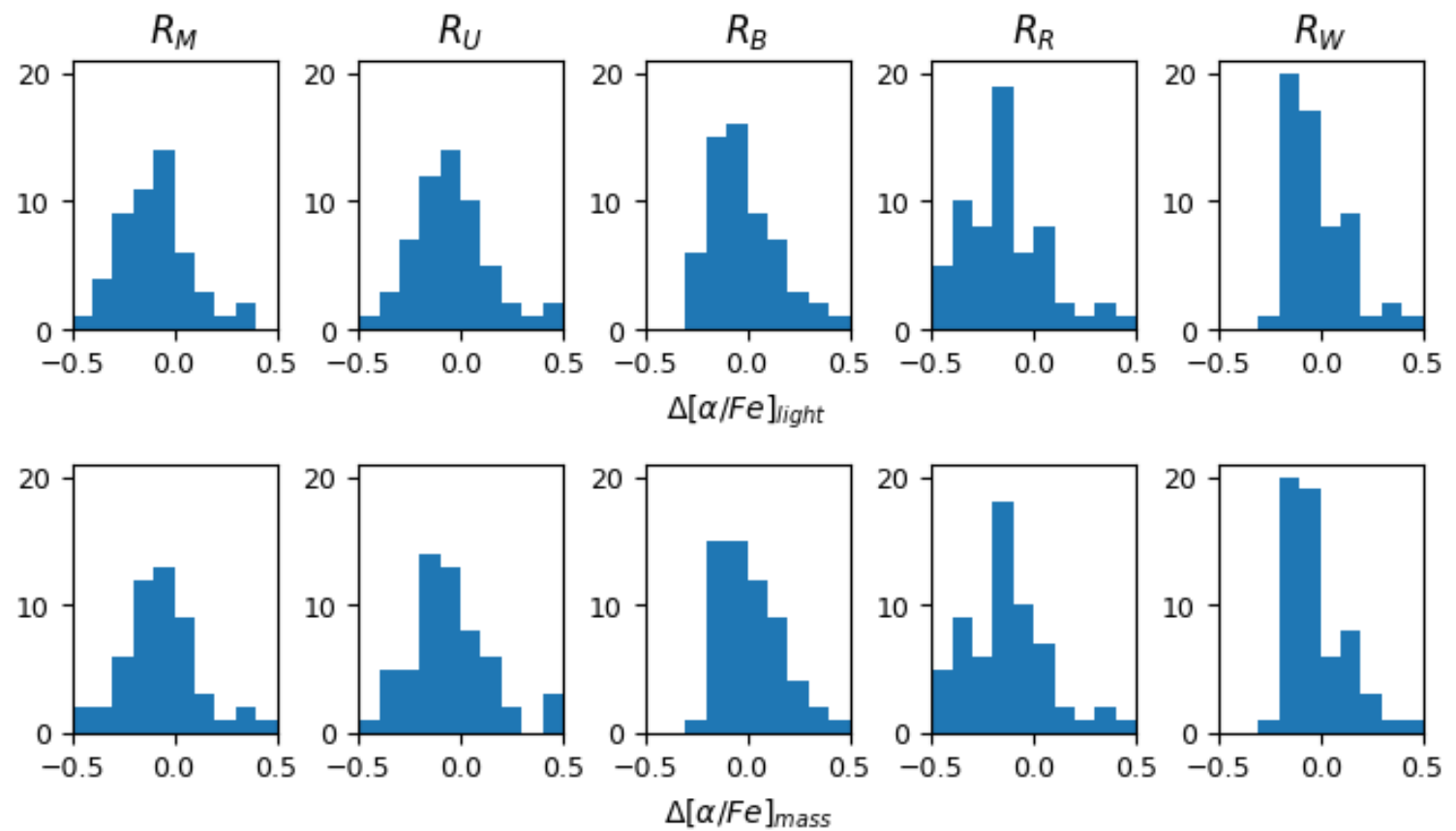

Figura 4.10: Distribuições de $\Delta[\alpha / \mathrm{Fe}]=[\alpha / \mathrm{Fe}]_{\text {Starlight }}-[\alpha / \mathrm{Fe}]_{\text {Reference. A linha superior mostra histogra- }}$ mas de $\Delta[\alpha / \mathrm{Fe}]$ ponderado por luz, enquanto a linha inferior mostra distribuições do mesmo parâmetro, porém ponderado por massa (Seção 4.3. Cada coluna representa um dos intervalos de comprimento de onda testados neste trabalho (Tabela 4.1). Crédito: Gonçalves et al. (submetido)

des (2010), os autores ajustam espectros de aglomerados globulares com o STARLight utilizando diversas bibliotecas de modelos e mostram que os resultados podem variar consideravelmente dependendo da escolha das SSPs.

\subsubsection{Incertezas devido ao SNR dos espectros}

Usando os valores de erro do fluxo disponíveis nos espectros da biblioteca WAGGS, criamos, para cada arquivo, vinte espectros perturbados. A cada valor de fluxo, em cada pixel, era somado seu valor de incerteza multiplicado por um valor aleatório de -1 a 1 (Equação 4.2). A seguir, todos os vinte espectros eram ajustados e a incerteza de cada parâmetro $P$ era dada pelo desvio padrão $\sigma_{P}$ dos vinte valores obtidos (Equação 4.3).

$$
\begin{aligned}
\text { flux }_{j}^{\text {perturbed }} & \rightarrow \text { flux }_{j}+\text { random }[-1,1] \times \operatorname{err}_{j} \\
\sigma_{P} & =\sqrt{\frac{\sum_{i=1}^{20}\left(P_{i}-\langle P\rangle\right)^{2}}{20}}
\end{aligned}
$$

Procurando otimizar tempo computacional, tal análise foi feita ajustando os espec- 
Tabela 4.2 - Desvio padrão típico dos parâmetros obtidos do ajuste de espéctros perturbados utilizando seus respectivos espectros de erro.

\begin{tabular}{ll}
\hline Parâmetro & mediana $(\sigma)$ \\
\hline $\log \left(t_{\text {light }}\right)$ & 0.002 \\
$\log \left(t_{\text {mass }}\right)$ & 0.002 \\
{$[\mathrm{Fe} / \mathrm{H}]_{\text {light }}$} & 0.02 \\
{$[\mathrm{Fe} / \mathrm{H}]_{\text {mass }}$} & 0.03 \\
{$[\alpha / \mathrm{Fe}]_{\text {light }}$} & 0.02 \\
{$[\alpha / \mathrm{Fe}]_{\text {mass }}$} & 0.02 \\
\hline
\end{tabular}

tros no intervalo $4828-5364 \AA\left(R_{W}\right)$ apenas. Os valores medianos para cada parâmetro (mostrados na Tabela 4.2) podem ser tomados como incertezas típicas para a biblioteca WAGGS, apesar de haver uma certa variação nos espectros individuais. Mas em todos os casos, tais simulações mostram que essas incertezas possuem valores relativamente baixos (os valores aglomerado a aglomerado são mostrados na Tabela C.1).

\subsubsection{Incertezas vindas de diferentes observações}

Uma outra fonte de erro pode ser a estratégia de observação e redução dos dados, que varia dependendo do telescópio e do instrumento utilizado.

De modo a testar essas possíveis variações, repetimos os ajustes no intervalo $R_{W}$, mas usando desta vez os espectros de luz integrada de aglomerados globulares de Schiavon et al. (2005). A Figura 4.11 mostra a comparação entre os parâmetros resultantes para os 35 aglomerados em comum entre as duas bibliotecas. Percebe-se que para os três parâmetros, há correlações entre os valores obtidos, com a maior parte dos resultados dos ajustes das duas bibliotecas apresentando diferenças menores que 0.1 dex para log(idade), 0.3 dex para $[\mathrm{Fe} / \mathrm{H}]$ e $0.1 \operatorname{dex}[\alpha / \mathrm{Fe}]$.

\subsection{Sumário de resultados}

As discussões apresentadas até aqui apontam para o fato de que há uma mudança significativa nos resultados quando variamos o intervalo espectral ajustado. Além disso, 

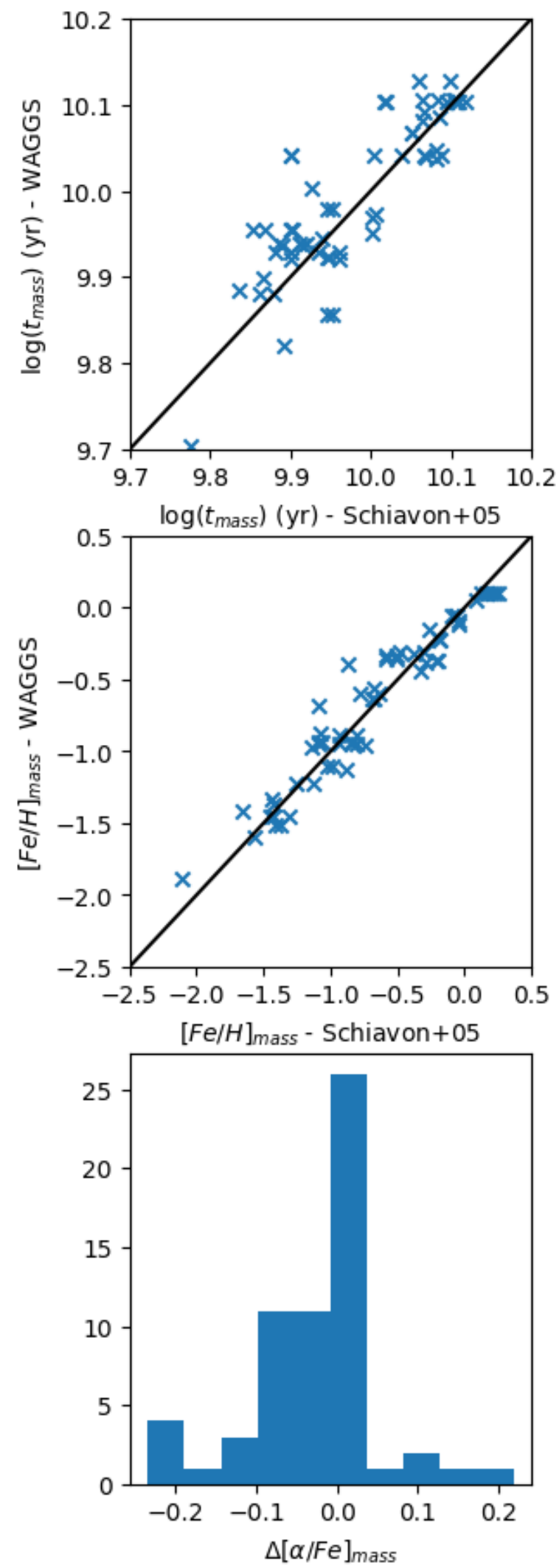

Figura 4.11: Valores de idade e $[\mathrm{Fe} / \mathrm{H}]$ (painéis superior e central, respectivamente) ponderados por massa resultantes dos ajustes feitos com o STARLight dos espectros da biblioteca WAGGS (eixo y) versus os resultados do mesmo processo, porém utilizando os espectros da biblioteca de Schiavon et al. (2005) para os aglomerados presentes em ambos os surveys. A linha $\mathrm{x}=\mathrm{y}$ é mostrada em preto. É notável que há uma alta correlação entre os valores de idade e de $[\mathrm{Fe} / \mathrm{H}]$. O painel inferior mostra a distribuição de $\Delta[\alpha / F e]=[\alpha / F e]_{\text {mass }, W A G G S}-[\alpha / F e]_{\text {mass,Schiavon }+05}$, onde é possível notar um pico em torno de zero. Crédito: Gonçalves et al. (submetido) 
Tabela 4.3 - Valores medianos de $\Delta$ parâmetros \pm intervalos interquartis (IQR) para cada intervalo de comprimento de onda. $\Delta P=P_{\text {Starlight }}-P_{\text {Reference }}$ onde $P$ representa cada parâmetro. Colunas $2-6$ indicam os intervalos ajustados, como definidos na Tabela 4.1 .

\begin{tabular}{|c|c|c|c|c|c|}
\hline Parâmetro & $R_{M}$ & $R_{U}$ & $R_{B}$ & $R_{R}$ & $R_{W}$ \\
\hline$\Delta \log \left(t_{\text {light }}\right)$ & $-0.44 \pm 0.45$ & $-0.27 \pm 0.23$ & $-0.40 \pm 0.41$ & $-0.56 \pm 0.41$ & $-0.19 \pm 0.29$ \\
$\Delta \log \left(t_{\text {mass }}\right)$ & $-0.19 \pm 0.34$ & $-0.19 \pm 0.16$ & $-0.06 \pm 0.17$ & $-0.17 \pm 0.18$ & $-0.14 \pm 0.22$ \\
$\Delta[\mathrm{Fe} / \mathrm{H}]_{\text {light }}$ & $0.33 \pm 0.28$ & $0.34 \pm 0.21$ & $0.24 \pm 0.20$ & $0.25 \pm 0.27$ & $0.18 \pm 0.24$ \\
$\Delta[\mathrm{Fe} / \mathrm{H}]_{\text {mass }}$ & $0.23 \pm 0.32$ & $0.39 \pm 0.29$ & $0.34 \pm 0.26$ & $0.09 \pm 0.39$ & $0.22 \pm 0.27$ \\
$\Delta[\alpha / \mathrm{Fe}]_{\text {light }}$ & $-0.10 \pm 0.19$ & $-0.05 \pm 0.22$ & $-0.05 \pm 0.23$ & $-0.17 \pm 0.22$ & $-0.05 \pm 0.17$ \\
$\Delta[\alpha / \mathrm{Fe}]_{\text {mass }}$ & $-0.08 \pm 0.20$ & $-0.06 \pm 0.22$ & $-0.02 \pm 0.22$ & $-0.14 \pm 0.23$ & $-0.05 \pm 0.17$ \\
$\Delta A_{V}$ & $0.10 \pm 0.30$ & $-0.25 \pm 0.50$ & $0.12 \pm 0.28$ & $0.31 \pm 0.40$ & $0.14 \pm 0.38$ \\
\hline
\end{tabular}

é importante ressaltar que usar o maior intervalo possível nem sempre retorna resultados mais fidedignos.

A Tabela 4.3 mostra as medianas e os intervalos interquartis (IQR) de cada $\Delta P=$ $P_{\text {Starlight }}-P_{\text {Reference, }}$, sendo $P$ qualquer um dos parâmetros ajustados. É notável que os valores de avermelhamento são melhores ajustados quando mais informação é fornecida: $R_{M}$ apresenta os menores valores de $\Delta A_{V}$. Para as idades, por outro lado, os valores se mostram mais próximos dos valores provenientes de ajustes de isócronas quando o ajuste é feito no intervalo $R_{B}$, e para o caso das idades ponderadas por massa.

No que diz respeito às abundâncias químicas, valores de $[\mathrm{Fe} / \mathrm{H}]$ mostram um desvio menor em $R_{R}$ (para valores ponderados por massa), enquanto $R_{W}$ retorna melhores ajustes de $[\alpha / \mathrm{Fe}]$ (não havendo uma diferença significativa entre os valores ponderados por luz e por massa). 
Capítulo 5

\section{Aplicação: bojos de galáxias do SDSS}

O método de ajuste espectral é mais comumente utilizado em espectros de galáxias, com o intuito de obter parâmetros de população estelar (idades e abundâncias químicas) médias desses objetos como um todo ou de seus componentes. Como discutido no capítulo anterior e mostrado na Figura 4.4 (para o caso das idades) e na Tabela 4.3, ajustes de objetos de metalicidade intermediária ou alta tendem a retornar valores consistentes para todos os parâmetros quando ajustados em $R_{W}$. Portanto, aplicamos a técnica descrita até aqui na amostra de galáxias apresentada em Gadotti (2009), cujos espectros das regiões centrais estão disponíveis no Sloan Digital Sky Survey.

A amostra contém galáxias elípticas e espirais (258 e 685 objetos, respectivamente). As espirais são sub-classificadas em bojo clássico, pseudo-bojo ou sem bojo (467, 202 e 16 objetos, respectivamente), podendo ou não conter barras ou núcleo ativo.

\subsection{Resultados do ajuste espectral}

As distribuições de $A_{V}$ ajustadas pelo Starlight são mostradas na Figura 5.1, separadas por tipo morfológico. Tais distribuições mostram um comportamento esperado: elípticas possuem valores mais baixos de $A_{V}$, o que é explicado pela menor quantidade de gás e poeira que esperamos nesse tipo de galáxia, enquanto os maiores valores aparecem em galáxias com pseudo-bojo, que podem possuir uma taxa de formação estelar não desprezível e presença de gás e poeira. Notamos também que as galáxias espirais barradas possuem, em média, valores de $A_{V}$ maiores que galáxias não barradas, o que pode ser uma evidência de que as barras de fato desempenham o papel de mover grandes quantidades de gás e poeira do disco em direção ao bojo. 


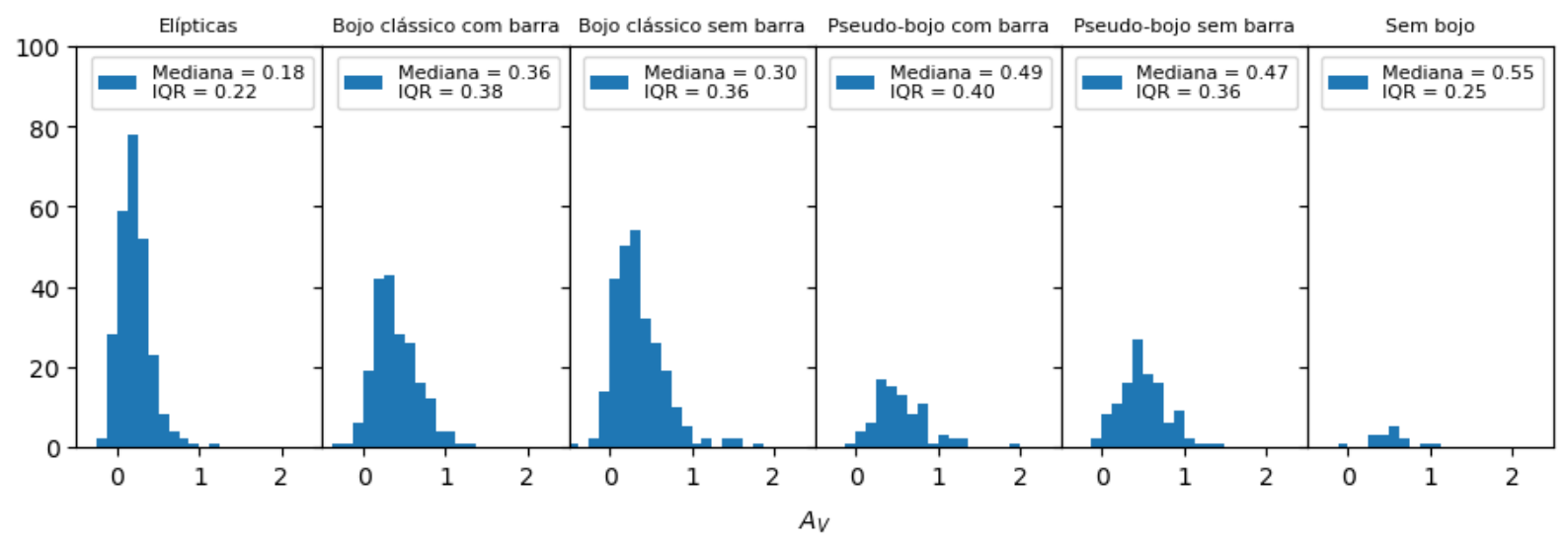

Figura 5.1: Distribuições de $A_{V}$ calculados pelo STARLight para os espectros das galáxias da amostra de Gadotti (2009) observados pelo SDSS. Diferentes paineis mostram resultados dos ajustes de diferentes morfologias. A média e a mediana de cada distribuição é mostrada na legenda de cada painel.

A Figura 5.2 mostra os valores calculados de idade, $[\mathrm{Fe} / \mathrm{H}]$ e $[\alpha / \mathrm{Fe}]$ ponderados por massa. Percebe-se que as elípticas e espirais com bojos clássicos são ajustadas com idades maiores e metalicidades mais altas, enquanto galáxias com pseudo-bojo tendem a apresentar valores mais uniformemente distribuídos.

Tais diferenças são esperadas, dado que bojos clássicos no geral possuem populações estelares muito semelhantes as de galáxias elípticas, sendo esses dois tipos de objeto resultados de grandes eventos de colisão. Já pseudo-bojos são resultado da evolução secular das galáxias, apresentando portanto uma população estelar mais próxima do que esperamos encontrar em discos, com taxas de formação estelar não desprezível (Kormendy, 2016).

\subsection{Comparação com a literatura}

Um subgrupo dessa amostra (essencialmente, as galáxias disco com bojo) foram estudadas em Coelho e Gadotti (2011), onde os autores discutem o impacto da presença de barras nas distribuições de idade das populações estelares, e portanto, nos processos de formação estelar em bojos galácticos. Eles concluem que existem diferenças significativas nas distribuições de idades de galáxias barradas e sem barra, mas que tais diferenças desaparecem quando apenas galáxias AGN são levadas em conta.

Como os autores fizeram os ajustes no intervalo que aqui chamamos de $R_{M}$, também ajustamos os espectros nessa faixa de comprimento de onda. Assim, a única diferença entre os dois casos é a escolha das bibliotecas de SSP. A seguir, comparamos os resultados. 

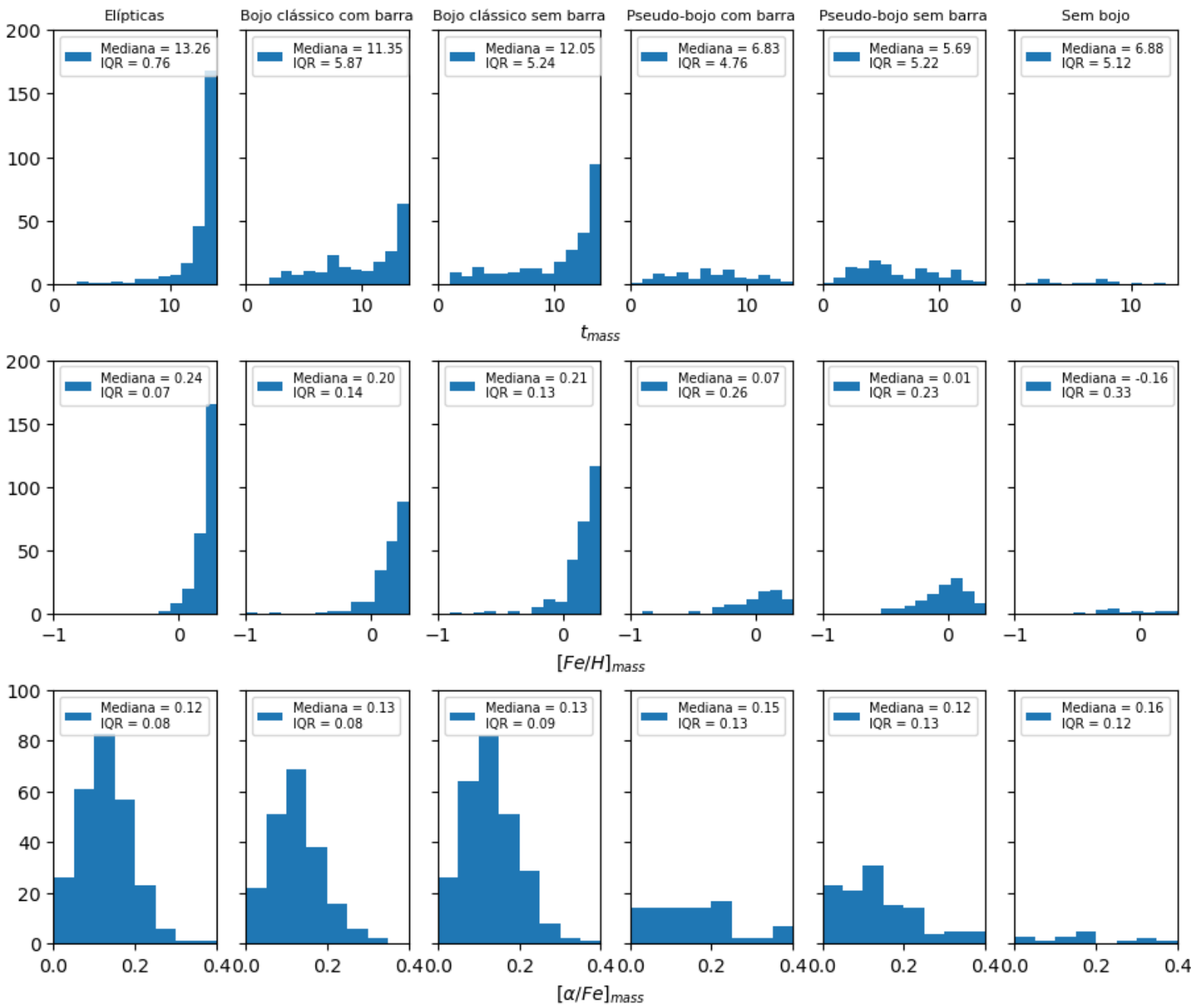

Figura 5.2: Parâmetroos de população estelar ajustados pelo STARLIGHT para os espectros das galáxias da amostra de Gadotti (2009) observados pelo SDSS. A linha superior apresenta distribuições de idade ponderada por massa, a linha central apresenta as distribuições de $[\mathrm{Fe} / \mathrm{H}]$ e a linha inferior apresenta distribuições de $[\alpha / \mathrm{Fe}]$. Diferentes colunas mostram resultados dos ajustes de diferentes morfologias. 

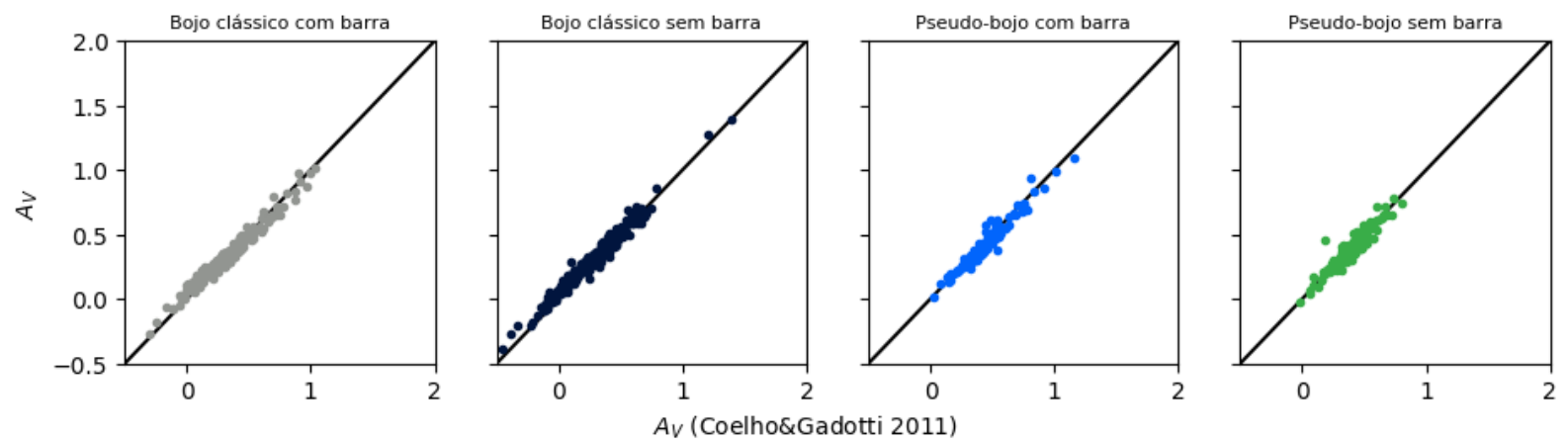

Figura 5.3: Valores de $A_{V}$ ajustados pelo Starlight para os espectros das galáxias da amostra de Gadotti (2009) observados pelo SDSS. O eixo y mostra os resultados deste trabalho, enquanto o eixo x mostra os resultados dos ajustes feitos em Coelho e Gadotti (2011). Diferentes colunas separam diferentes morfologias. A linha $\mathrm{x}=\mathrm{y}$ é mostrada em preto.
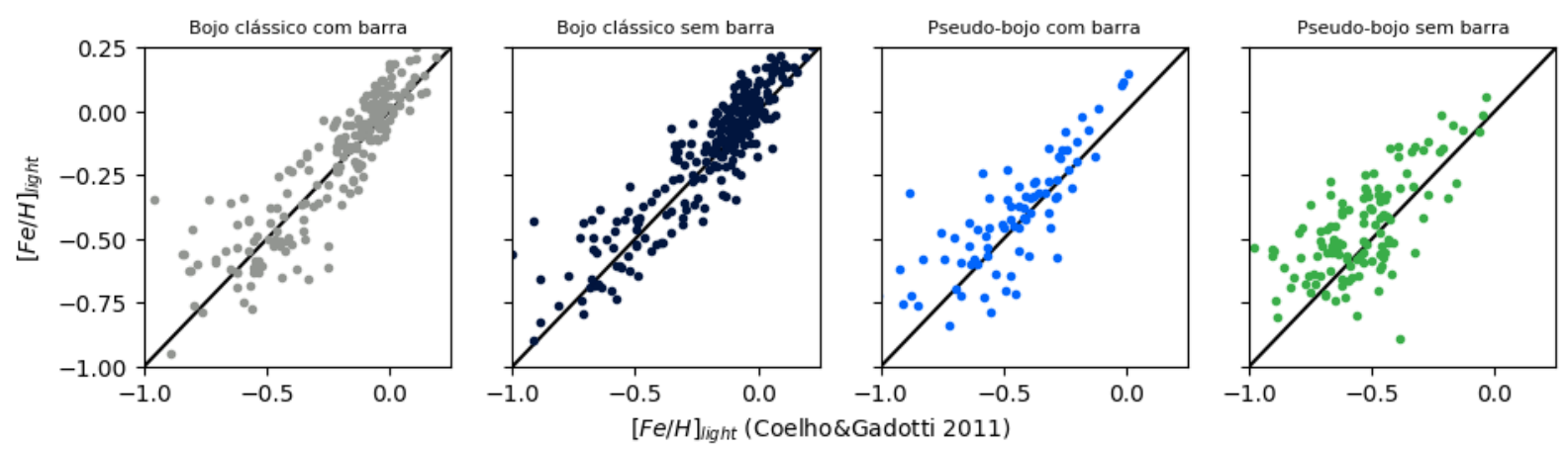

Figura 5.4: Valores de $[\mathrm{Fe} / \mathrm{H}]_{\text {light }}$ ajustados pelo StARLIGHT para os espectros das galáxias da amostra de Gadotti (2009) observados pelo SDSS. O eixo y mostra os resultados deste trabalho, enquanto o eixo x mostra os resultados dos ajustes feitos em Coelho e Gadotti (2011). Diferentes colunas separam diferentes morfologias. A linha $\mathrm{x}=\mathrm{y}$ é mostrada em preto.

Os valores de $A_{V}$ calculados pelos autores mostram uma alta correlação com os valores ajustados neste trabalho, como mostra a Figura 5.3 .

$[\mathrm{Fe} / \mathrm{H}]_{\text {light }}$ (Figura 5.4) mostra um espalhamento em torno da linha $\mathrm{x}=\mathrm{y}$ (por volta de $\sim 0.3$ dex). Para $t_{\text {light }}$ (Figura 5.5), os valores mostram uma alta correlação.

Uma possível hipótese para as diferenças (espalhamento em torno da linha $\mathrm{x}=\mathrm{y}$ ) entre os valores deste estudo e o de Coelho e Gadotti (2011) é o uso de diferentes modelos: os autores utilizaram a biblioteca de SSPs de Vazdekis et al. (2010), que é feita baseada numa biblioteca de espectros estelares empírica, enquanto utilizamos a de Vazdekis et al. (2015), que além de uma biblioteca empírica, também utiliza espectros sintéticos (o que permite a criação de modelos com $[\alpha / \mathrm{Fe}]$ diferente de 0.0$)$. 

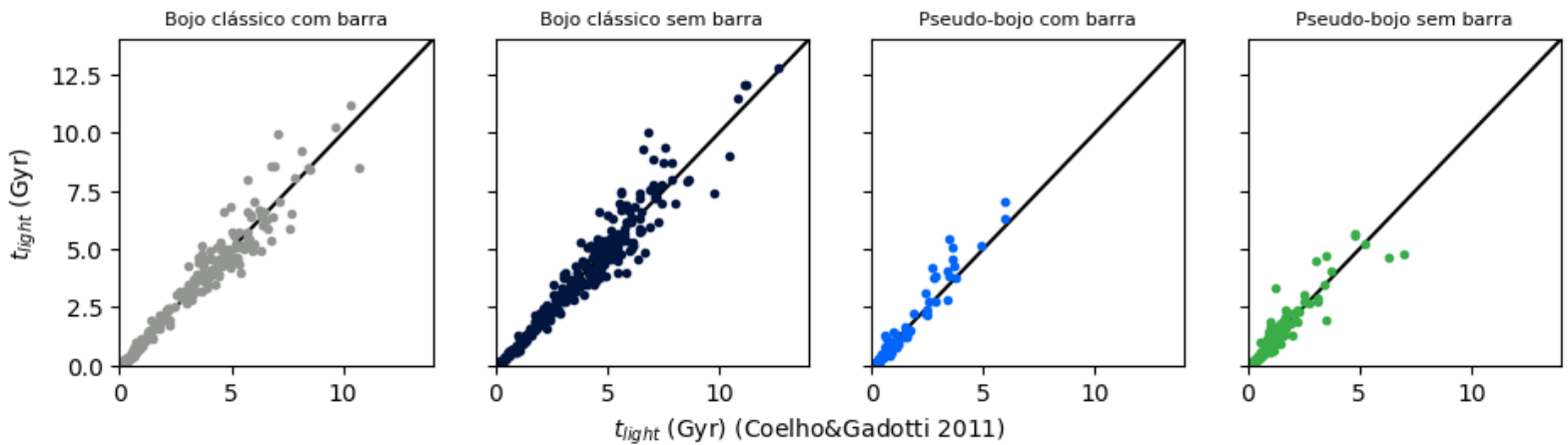

Figura 5.5: Valores de $t_{\text {light }}$ ajustados pelo Starlight para os espectros das galáxias da amostra de Gadotti (2009) observados pelo SDSS. O eixo y mostra os resultados deste trabalho, enquanto o eixo x mostra os resultados dos ajustes feitos em Coelho e Gadotti (2011). Diferentes colunas separam diferentes morfologias. A linha $\mathrm{x}=\mathrm{y}$ é mostrada em preto.

\subsection{Correlações com morfologia}

Nesta seção investigamos a presença (ou não) de correlações entre os aspectos morfológicos das galáxias e nossos resultados de população estelar para suas regiões centrais. Utilizamos os valores disponíveis em Gadotti (2009) para os parâmetros morfológicos desses objetos (ver Tabela 2.3) e os valores de $A_{V}$, idade, $[\mathrm{Fe} / \mathrm{H}]$ e $[\alpha / \mathrm{Fe}]$ resultantes de ajustes feitos com o Starlight. Para tal análise, empregamos o cálculo de coeficientes de correlação de Pearson (Pearson, 1900).

O coeficiente de correlação de Pearson, representado por $r$, é aplicado em variáveis quantitativas para medir o grau de correlação linear entre elas. O coeficiente $r$ é dado por

$$
r=\frac{\sum\left(x_{i}-x_{\text {médio }}\right)\left(y_{i}-y_{\text {médio }}\right)}{\sqrt{\left(\sum\left(x_{i}-x_{\text {médio }}\right)^{2}\right)\left(\sum\left(y_{i}-y_{\text {médio }}\right)^{2}\right)}}
$$

onde x e y são as duas variáveis entre as quais se deseja calcular a correlação. $r$ varia de -1 a 1, com valores negativos indicando correlação negativa (ou anti-correlação) e valores positivos indicando correlação positiva.

Os limites do que se considera uma correlação forte não são consenso em estudos estatísticos. Nesse trabalho, usaremos as definições dadas em Callegari-Jacques (2003):

- Se $0 \leqslant|r|<0.3$, a correlação linear é fraca.

- Se $0.3 \leqslant|r|<0.6$, a correlação linear é moderada.

- Se $0.6 \leqslant|r|<0.9$, a correlação linear é forte. 
Tabela 5.1 - Pares de parâmetros (morfologia/população estelar) que mostram correlações moderadas. As colunas representam os quatro grandes grupos morfológicos estudados (elípticas $E$ e dois tipos de espirais $S p)$, cada linha representa um dos quatro parâmetros de população estelar $\left(A_{V}\right.$, idade, $\left.[\mathrm{Fe} / \mathrm{H}] \mathrm{e}[\alpha / \mathrm{Fe}]\right)$. Cada célula contém o parâmetro morfológico que apresenta correlações moderadas com o parâmetro de população estelar em questão. Os parâmetros morfológicos são listados e descritos na tabela 2.3 .

\begin{tabular}{|c|c|c|c|}
\hline & $\mathbf{E}$ & $S p$ bojo clássico & $S p$ pseudo-bojo \\
\hline$A_{V}$ & - & $\begin{array}{l}\mu_{e, b a r} ; \quad \epsilon_{b a r} ; \quad n_{b a r} ; \mathrm{B} / \mathrm{T} ; \\
\mathrm{D} / \mathrm{T} ; M / L_{d i s k}\end{array}$ & $\mathrm{~h} ; M / L_{\text {bulge }} ; M / L_{b a r}$ \\
\hline Idade & $M / L_{\text {bulge }}$ & $\begin{array}{l}M / L_{\text {bulge }} ; \quad M / L_{\text {disk }} ; \\
M_{\text {bulge }}\end{array}$ & $\begin{array}{l}\mathrm{h} ; \quad \mu_{e, b a r} ; \quad r_{e, b a r} ; \quad \epsilon_{\text {bar }} ; \\
n_{\text {bar }} ; \mathrm{c} ; \operatorname{Bar} / \mathrm{T} ; M / L_{\text {bulge }} ; \\
M / L_{\text {disk }}\end{array}$ \\
\hline$[\mathrm{Fe} / \mathbf{H}]$ & $r_{e} ; M / L_{\text {bulge }} ; M_{\text {bulge }}$ & 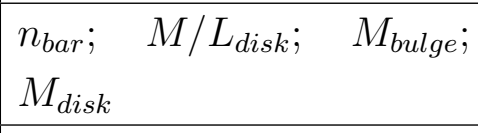 & $\begin{array}{l}\mathrm{h} ; r_{e} ; r_{e, b a r} ; \epsilon_{b a r} ; n_{b a r} ; \mathrm{c} ; \\
\mathrm{Bar} / \mathrm{T} ; M / L_{b a r}\end{array}$ \\
\hline$[\alpha / \mathbf{F e}]$ & $M_{\text {bulge }}$ & $\begin{array}{l}\mathrm{h} ; \mu_{e, b a r} ; \mu_{e} ; L_{b a r} ; \mathrm{B} / \mathrm{T} ; \\
\mathrm{D} / \mathrm{T} ; \operatorname{Bar} / \mathrm{T}\end{array}$ & $\begin{array}{l}\mu_{0} ; \mathrm{n} ; \mu_{e, b a r} ; \mathrm{B} / \mathrm{T} ; \mathrm{D} / \mathrm{T} ; \\
M / L_{\text {disk }} ; M / L_{b a r}\end{array}$ \\
\hline
\end{tabular}

- Se $0.9 \leqslant|r|<1$, a correlação linear é muito forte.

Calculamos os coeficientes de correlação para todos os parâmetros (morfológicos e de população estelar). As galáxias foram divididas em grupos dependendo de sua morfologia (elípticas, espirais com bojo clássico, espirais com pseudo-bojo, espirais sem bojo) e também pela presença ou não de barras e AGNs.

Considerando correlações moderadas e fortes $(0.3 \leqslant|r|<0.6)$, encontramos algumas possíveis correlações entre parâmetros morfológicos das galáxias e as populações estelares de suas partes centrais. Esses pares de parâmetros são listados na Tabela 5.1. As correlações mais fortes (maiores valores de $|r|$ ) foram encontradas para galáxias AGN com pseudo-bojo e não barradas, conforme ilustrado na matriz de correlações da Figura 5.6 . Idades mostram correlações fortes com o raio efetivo do bojo $(r=0.75)$ e com a massa do bojo $(r=0.72)$, e uma anti-correlação forte com a razão massa-luminosidade do bojo $(r=-0.69)$.

As correlações mostram que bojos maiores e mais massivos tendem a ter populações mais velhas, o que pode ser resultado do fato de que tais objetos tiveram mais tempo para "crescer" (seja por formação estelar in situ ou por acreção). A anti-correlação com massa-luminosidade é esperada: populações mais velhas possuem M/L menores. O que não fica claro é porque tais correlações e anti-correlação aparecem mais fortemente em uma morfologia específica (no caso pseudo-bojo sem barras), e qual o papel do AGN nesses 
resultados.

Valores de $[\alpha / \mathrm{Fe}]$ mostram uma correlação forte com o raio efetivo do bojo $(r=0.65)$ e com a razão luminosidade do bojo-luminosidade total $(r=0.65)$ e anti-corrrelações fortes com a razão luminosidade do disco-luminosidade total $(r=-0.65)$ e com a razão massa-luminosidade do bojo $(r=-0.7)$.

As demais matrizes de correlação são mostradas no apêndice D. As implicações dessas possíveis correlações serão melhor investigadas em um trabalho futuro. 


\section{Pseudo bojo sem barra, AGN}

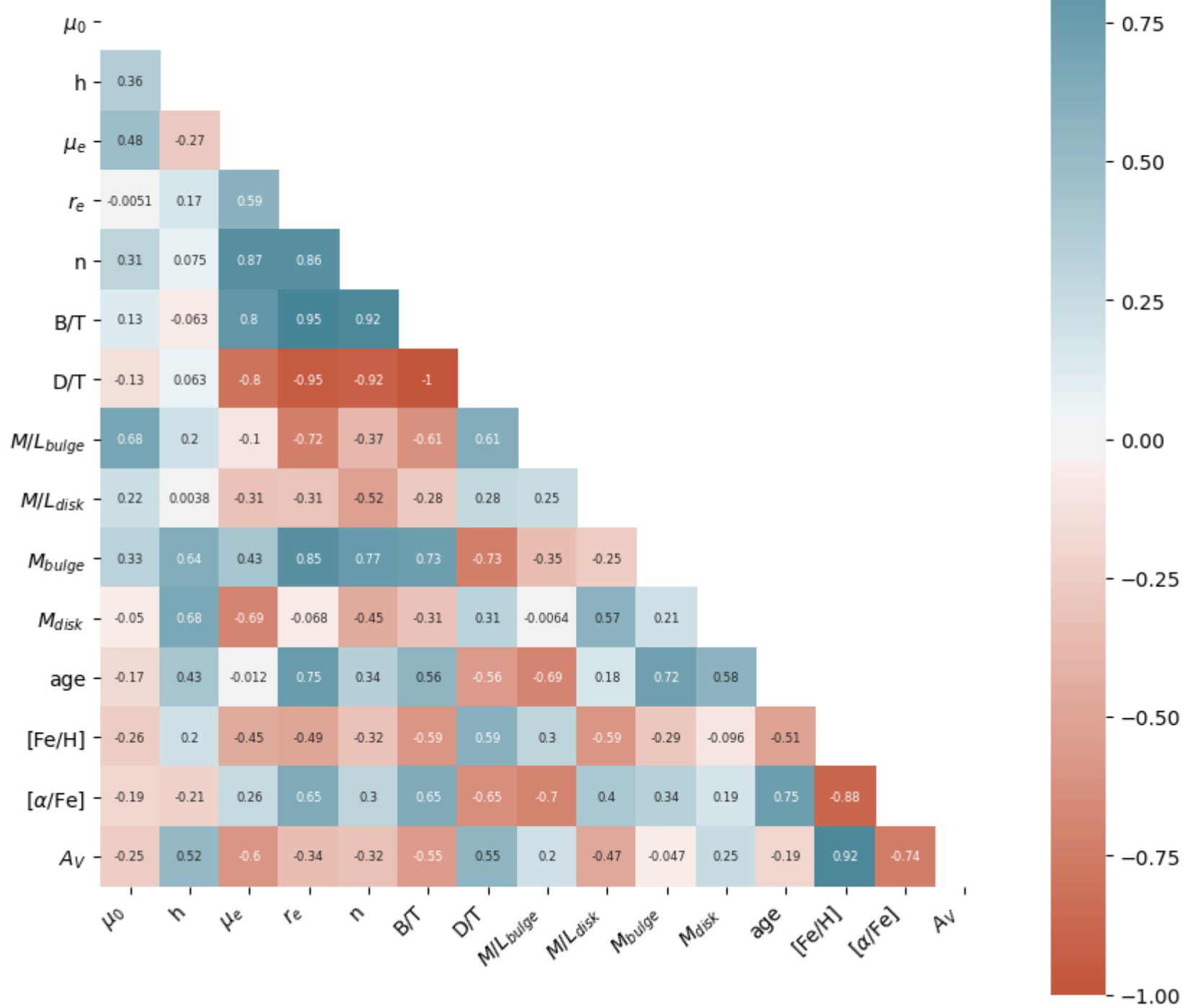

Figura 5.6: Matriz de correlações para todos os parâmetros (morfológicos - mostrados na Tabela 2.3 e de população estelar - calculados com o StARLight) para galáxias AGN com pseudo bojo sem barra da amostra de Gadotti (2009). Os números em cada célula representam o coeficiente de correlação de Pearson $r$ entre os parâmetros. Tons de azul representam correlações positivas, enquanto tons de vermelho representam correlações negativas. 


\section{Capítulo 6}

\section{Conclusões}

Neste trabalho, obtivemos valores de $A_{V}$, idades, $[\mathrm{Fe} / \mathrm{H}]$ e $[\alpha / \mathrm{Fe}]$ através de ajuste espectral de espectros de luz integrada de aglomerados globulares (Usher et al., 2017), simulando um processo que é comumente utilizado em espectros de galáxias. Repetimos a análise para diferentes intervalos de comprimento de onda, e comparamos os resultados com valores de referência compilados da literatura (obtidos de análises de CMDs e de espectroscopia de alta resolução) de modo a avaliar quais intervalos retornam resultados mais fiduciais. Nossas conclusões são apresentadas abaixo:

- Para todos os parâmetros, a mudança do intervalo de comprimento de onda ajustado acarreta em mudanças nos parâmetros resultantes.

- Avermelhamento é o único parâmetro favorecido pelo uso do maior intervalo possível, contrariando o senso comum de "quanto mais, melhor" existente na comunidade científica que utiliza o método. $[\alpha / \mathrm{Fe}]$, como contrapartida, foi melhor ajustado com o menor intervalo testado neste trabalho.

- Os intervalos que favorecem idades, $[\mathrm{Fe} / \mathrm{H}]$ e $[\alpha / \mathrm{Fe}]$ são diferentes $\left(R_{B} 4170-5540\right.$, $R_{R} 5280-7020$ e $R_{W}$ 4828-5364, respectivamente).

- Objetos pobres em metais retornam idades muito discrepantes com os valores resultantes de ajustes de isócrona. A correlação entre os resíduos de idade (ajustadas - valores da literatura) com a metalicidade pode ser um indicador dos efeitos de estrelas de ramo horizontal extendido ou blue stragglers, já discutidos na literatura. Além disso, o fato de os modelos serem feitos utilizando bibliotecas estelares esparçamente populadas em regiões de baixa metalicidade pode também ter um papel no que observamos (Coelho et al., 2020). 
As explicações para nossas conclusões podem ter diversas origens, incluindo a escolha dos modelos (Vazdekis et al., 2015) ou código (Cid Fernandes et al., 2005) utilizados. Porém, reiteramos que nossos resultados são qualitativamente similares a outros trabalhos, que utilizaram diferentes códigos e modelos (Walcher et al., 2009; Cezario et al., 2013, por exemplo). Assim, concluímos que há algo mais fundamental por trás dos efeitos observados, e que usuários de algoritmos de ajuste espectral analisando espectros integrados de galáxias não obterão os melhores resultados utilizando necessariamente mais informação (maiores intervalos de comprimento de onda).

Aplicamos então a técnica em uma amostra de galáxias do Universo Local, ajustando espectros SDSS de suas regiões centrais. Os parâmetros de população estelar resultantes mostram que galáxias elípticas e bojos clássicos de espirais tendem a ter populações mais velhas e mais metálicas, quando comparadas a pseudo-bojos e galáxias sem bojo. Além disso, as distribuições de $A_{V}$ possuem picos em valores mais altos para galáxias barradas, o que pode indicar que barras aumentam a concentração de gás e poeira nas regiões centrais das galáxias. Por fim, correlacionamos esses resultados com parâmetros morfológicos. Algumas correlações fortes foram encontradas para uma das morfologias (galáxias não barradas com pseudo-bojo e AGN), além de diversas correlações moderadas em quase todos os tipos morfológicos.

\section{Perspectivas}

Este trabalho faz uma análise de confiabilidade do método de ajuste espectral pixela-pixel, utilizando o software StARLight e os modelos de população estelar simples de Vazdekis et al. (2015), com foco nas mudanças causadas nos parâmetros resultados devido à escolha do intervalo de comprimento de onda do ajuste.

Um dos resultados apresentados é um conjunto de intervalos espectrais que, em nossos testes, retornaram resultados mais próximos dos valores de referência. Porém, tais intervalos são altamente dependentes do instrumento utilizado na observação dos espectros

de aglomerados globulares da biblioteca WAGGS (Usher et al., 2017), conjunto de dados utilizado em grande parte da análise.

Uma possível maneira de refinar tais resultados seria isolar as regiões espectrais específicas responsáveis pelos resultados (observamos, por exemplo, que o melhor intervalo 
para o ajuste das idades contém $H \beta$ e $H \gamma$, mas não $H \alpha$ ou $H \delta$, e que o melhor intervalo para $[\alpha / \mathrm{Fe}]$ contém o tripleto de Magnésio. Estudos da sensibilidade de diversas regiões espectrais a elementos químicos já foram feitos extensivamente na literatura, através de trabalhos com índices espectrais. No entanto, ainda faltam algumas diretrizes mais específicas em como melhor usar esse conhecimento em ajustes espectrais pixel-a-pixel, onde a comunidade tende a favorecer usar todo o intervalo espectral observado. Identificar e assegurar tais dependências nos permitiria entender quais características específicas afetam diretamente os resultados quando o método de ajuste espectral é utilizado. 


\section{Referências Bibliográficas}

Abazajian K., Adelman-McCarthy J. K., Agüeros M. A., Allam S. S., Anderson The Second Data Release of the Sloan Digital Sky Survey, AJ, 2004, vol. 128, p. 502

Abel T., Bryan G. L., Norman M. L., The Formation of the First Star in the Universe, Science, 2002, vol. 295, p. 93

Alves-Brito A., Forbes D. A., Mendel J. T., Hau G. K. T., Murphy M. T., The outer halo globular clusters of M31, MNRAS, 2009, vol. 395, p. L34

Athanassoula E., Formation and Dynamical Evolution of Galaxies and of their Components. In Planetary Nebulae as Astronomical Tools, vol. 804 of American Institute of Physics Conference Series, 2005, p. 333

Baade W., NGC 147 and NGC 185, Two New Members of the Local Group of Galaxies, ApJ, 1944, vol. 100, p. 147

Barbuy B., Bica E., Ortolani S., Globular clusters within 5(deg) of the Galactic center, A\&A, 1998, vol. 333, p. 117

Barbuy B., Chiappini C., Cantelli E., Depagne E., Pignatari M., Hirschi R., Cescutti G., Ortolani S., Hill V., Zoccali M., Minniti D., Trevisan M., Bica E., Gómez A., Highresolution abundance analysis of red giants in the globular cluster NGC 6522, A\&A, 2014, vol. 570, p. A76

Barbuy B., Muniz L., Ortolani S., Ernandes H., Dias B., Saviane I., Kerber L., Bica E., Pérez-Villegas A., Rossi L., Held E. V., High-resolution abundance analysis of four red giants in the globular cluster NGC 6558[U+2605], A\&A, 2018, vol. 619, p. A178 
Barbuy B., Zoccali M., Ortolani S., Hill V., Minniti D., Bica E., Renzini A., Gómez A., VLT-FLAMES analysis of 8 giants in the bulge metal-poor globular cluster NGC 6522: oldest cluster in the Galaxy?. Analysis of 8 giants in NGC 6522, A\&A, 2009, vol. 507, p. 405

Bastian N., Lardo C., Multiple Stellar Populations in Globular Clusters, ARA\&A, 2018, vol. 56 , p. 83

Bastian N., Silva-Villa E., Constraints on possible age spreads within young massive clusters in the Large Magellanic Cloud, MNRAS, 2013, vol. 431, p. L122

Beers T. C., Christlieb N., The Discovery and Analysis of Very Metal-Poor Stars in the Galaxy, ARA\&A, 2005, vol. 43, p. 531

Bica E., Alloin D., Analysis of absorption-line spectra in a sample of 164 galactic nuclei., A\&AS, 1987, vol. 70, p. 281

Boberg O. M., Friel E. D., Vesperini E., Chemical Abundances in NGC 5024 (M53): A Mostly First Generation Globular Cluster, ApJ, 2016, vol. 824, p. 5

Bragaglia A., Carretta E., Sollima A., Donati P., D’Orazi V., Gratton R. G., Lucatello S., Sneden C., NGC 6139: a normal massive globular cluster, or a first-generation dominated cluster? Clues from the light elements, A\&A, 2015, vol. 583, p. A69

Bromm V., Coppi P. S., Larson R. B., Forming the First Stars in the Universe: The Fragmentation of Primordial Gas, The Astrophysical Journal, 1999, vol. 527, p. L5

Bruzual G., Charlot S., Stellar population synthesis at the resolution of 2003, MNRAS, 2003, vol. 344, p. 1000

Bruzual A. G., Charlot S., Spectral Evolution of Stellar Populations Using Isochrone Synthesis, ApJ, 1993, vol. 405, p. 538

Callegari-Jacques S., Bioestatística: princípios e aplicações. Artmed, 2003

Carretta E., Bragaglia A., Gratton R., Lucatello S., Na-O anticorrelation and HB. VIII. Proton-capture elements and metallicities in 17 globular clusters from UVES spectra, A\&A, 2009, vol. 505, p. 139 
Carretta E., Bragaglia A., Gratton R. G., D'Orazi V., Lucatello S., Momany Y., Sollima A., Bellazzini M., Catanzaro G., Leone F., The extreme chemistry of multiple stellar populations in the metal-poor globular cluster NGC 4833, A\&A, 2014, vol. 564, p. A60

Carretta E., Bragaglia A., Gratton R. G., D'Orazi V., Lucatello S., Sollima A., Momany Y., Catanzaro G., Leone F., The normal chemistry of multiple stellar populations in the dense globular cluster NGC 6093 (M 80), A\&A, 2015, vol. 578, p. A116

Carretta E., Bragaglia A., Gratton R. G., Lucatello S., Bellazzini M., Catanzaro G., Leone F., Momany Y., Piotto G., D'Orazi V., Detailed abundances of a large sample of giant stars in M 54 and in the Sagittarius nucleus, A\&A, 2010, vol. 520, p. A95

Carretta E., Bragaglia A., Gratton R. G., Lucatello S., D’Orazi V., Bellazzini M., Catanzaro G., Leone F., Momany Y., Sollima A., VizieR Online Data Catalog: Detailed chemical abundances in the GlC NGC 362 (Carretta+ 2013), VizieR Online Data Catalog, 2013, vol. 355

Carretta E., Bragaglia A., Lucatello S., D’Orazi V., Gratton R. G., Donati P., Sollima A., Sneden C., Chemical characterisation of the globular cluster NGC 5634 associated to the Sagittarius dwarf spheroidal galaxy, A\&A, 2017, vol. 600, p. A118

Carretta E., Lucatello S., Gratton R. G., Bragaglia A., D’Orazi V., Multiple stellar populations in the globular cluster NGC 1851, A\&A, 2011, vol. 533, p. A69

Cezario E., Coelho P. R. T., Alves-Brito A., Forbes D. A., Brodie J. P., Full spectral fitting of Milky Way and M 31 globular clusters: ages and metallicities, A\&A, 2013, vol. 549, p. A60

Childress M. J., Vogt F. P. A., Nielsen J., Sharp R. G., PyWiFeS: a rapid data reduction pipeline for the Wide Field Spectrograph (WiFeS), Ap\&SS, 2014, vol. 349, p. 617

Chilingarian I., Prugniel P., Sil'Chenko O., Koleva M., NBursts: Simultaneous Extraction of Internal Kinematics and Parametrized SFH from Integrated Light Spectra. In Stellar Populations as Building Blocks of Galaxies, vol. 241 of IAU Symposium, 2007, p. 175

Cid Fernandes R., Paleontology of Galaxies: Recovering the Star Formation Chemical Enrichment Histories from Galaxy Spectra, ArXiv Astrophysics e-prints, 2007 
Cid Fernandes R., Mateus A., Sodré L., Stasińska G., Gomes J. M., Semi-empirical analysis of Sloan Digital Sky Survey galaxies - I. Spectral synthesis method, MNRAS, 2005, vol. 358 , p. 363

Cid Fernandes R., On tests of full spectral fitting algorithms, Monthly Notices of the Royal Astronomical Society, 2018, vol. 480, p. 4480

Coelho P., Barbuy B., Meléndez J., Schiavon R. P., Castilho B. V., A library of high resolution synthetic stellar spectra from $300 \mathrm{~nm}$ to $1.8 \mu \mathrm{m}$ with solar and $\alpha$-enhanced composition, A\&A, 2005, vol. 443, p. 735

Coelho P., Bruzual G., Charlot S., Weiss A., Barbuy B., Ferguson J. W., Spectral models for solar-scaled and alpha-enhanced stellar populations, MNRAS, 2007, vol. 382, p. 498

Coelho P., Gadotti D. A., Bars Rejuvenating Bulges? Evidence from Stellar Population Analysis, ApJ, 2011, vol. 743, p. L13

Coelho P., Mendes de Oliveira C., Cid Fernandes R., An analysis of the composite stellar population in M32, MNRAS, 2009, vol. 396, p. 624

Coelho P. R. T., A new library of theoretical stellar spectra with scaled-solar and alphaenhanced mixtures, MNRAS, 2014, vol. 440, p. 1027

Coelho P. R. T., Bruzual G., Charlot S., To use or not to use synthetic stellar spectra in population synthesis models?, MNRAS, 2020, vol. 491, p. 2025

Cohen J. G., Meléndez J., Abundances in a Large Sample of Stars in M3 and M13, AJ, 2005, vol. 129 , p. 303

Colucci J. E., Bernstein R. A., Cameron S. A., McWilliam A., Globular Cluster Abundances from High-resolution, Integrated-light Spectroscopy. IV. The Large Magellanic Cloud: alpha, Fe-peak, Light, and Heavy Elements, ApJ, 2012, vol. 746, p. 29

Colucci J. E., Bernstein R. A., McWilliam A., Globular Cluster Abundances from Highresolution, Integrated-light Spectroscopy. II. Expanding the Metallicity Range for Old Clusters and Updated Analysis Techniques, ApJ, 2017, vol. 834, p. 105 
Conroy C., Graves G. J., van Dokkum P. G., Early-type Galaxy Archeology: Ages, Abundance Ratios, and Effective Temperatures from Full-spectrum Fitting, ApJ, 2014, vol. 780 , p. 33

Conroy C., Villaume A., van Dokkum P. G., Lind K., Metal-rich, Metal-poor: Updated Stellar Population Models for Old Stellar Systems, ApJ, 2018, vol. 854, p. 139

De Angeli F., Piotto G., Cassisi S., Busso G., Recio-Blanco A., Salaris M., Aparicio A., Rosenberg A., Galactic Globular Cluster Relative Ages, AJ, 2005, vol. 130, p. 116

de Boer T. J. L., Fraser M., Four and one more: The formation history and total mass of globular clusters in the Fornax dSph, A\&A, 2016, vol. 590, p. A35

de Souza R. E., Gadotti D. A., dos Anjos S., BUDDA: A New Two-dimensional Bulge/Disk Decomposition Code for Detailed Structural Analysis of Galaxies, ApJS, 2004, vol. 153, p. 411

Delgado R. M. G., Fernandes R. C., Testing spectral models for stellar populations with star clusters - II. Results, Monthly Notices of the Royal Astronomical Society, 2010, vol. 403, p. 797

Dickens R. J., Croke B. F. W., Cannon R. D., Bell R. A., Evidence from stellar abundances for a large age difference between two globular clusters, Nature, 1991, vol. 351, p. 212

Dirsch B., Richtler T., Gieren W. P., Hilker M., Age and metallicity for six LMC clusters and their surrounding field population, A\&A, 2000, vol. 360, p. 133

Dopita M., Hart J., McGregor P., Oates P., Bloxham G., Jones D., The Wide Field Spectrograph (WiFeS), Ap\&SS, 2007, vol. 310, p. 255

Dopita M., Rhee J., Farage C., McGregor P., Bloxham G., Green A., Roberts B., Neilson J., Wilson G., Young P., Firth P., Busarello G., Merluzzi P., The Wide Field Spectrograph (WiFeS): performance and data reduction, Ap\&SS, 2010, vol. 327, p. 245

Dotter A., Chaboyer B., Jevremović D., Baron E., Ferguson J. W., Sarajedini A., Anderson J., The ACS Survey of Galactic Globular Clusters. II. Stellar Evolution Tracks, Isochrones, Luminosity Functions, and Synthetic Horizontal-Branch Models, The Astronomical Journal, 2007, vol. 134, p. 376 
Dotter A., Sarajedini A., Anderson J., Globular Clusters in the Outer Galactic Halo: New Hubble Space Telescope/Advanced Camera for Surveys Imaging of Six Globular Clusters and the Galactic Globular Cluster Age-metallicity Relation, ApJ, 2011, vol. 738, p. 74

Dotter A., Sarajedini A., Anderson J., Aparicio A., Bedin L. R., Chaboyer B., Majewski S., Marín-Franch A., Milone A., Paust N., Piotto G., Reid I. N., Rosenberg A., Siegel M., The ACS Survey of Galactic Globular Clusters. IX. Horizontal Branch Morphology and the Second Parameter Phenomenon, ApJ, 2010, vol. 708, p. 698

Faber S. M., Friel E. D., Burstein D., Gaskell C. M., Old stellar populations. II. an analysis of K-giant spectra., ApJS, 1985, vol. 57, p. 711

Feltzing S., Primas F., Johnson R. A., Stellar abundances and ages for metal-rich Milky Way globular clusters. Stellar parameters and elemental abundances for 9 HB stars in NGC 6352, A\&A, 2009, vol. 493, p. 913

Fernandes R. C., Delgado R. M. G., Testing spectral models for stellar populations with star clusters - I. Methodology, Monthly Notices of the Royal Astronomical Society, 2010, vol. 403, p. 780

Filler D., Ivans I. I., Simmerer J., Galactic Globular Cluster NGC1261: "Young", Lowalpha and Star-to-Star Abundance Variations In Na-O?. In American Astronomical Society Meeting Abstracts \#219 , vol. 219 of American Astronomical Society Meeting Abstracts, 2012, p. 152.05

Gadotti D. A., Structural properties of pseudo-bulges, classical bulges and elliptical galaxies: a Sloan Digital Sky Survey perspective, MNRAS, 2009, vol. 393, p. 1531

Gallazzi A., Charlot S., Brinchmann J., White S. D. M., Tremonti C. A., The ages and metallicities of galaxies in the local universe, MNRAS, 2005, vol. 362, p. 41

Glatt K., Gallagher III J. S., Grebel E. K., Nota A., Sabbi E., Sirianni M., Clementini G., Tosi M., Harbeck D., Koch A., Cracraft M., An Accurate Age Determination for the Small Magellanic Cloud Star Cluster NGC 121 with the Hubble Space Telescope/Advanced Camera for Surveys, AJ, 2008, vol. 135, p. 1106 
Glatt K., Grebel E. K., III J. S. G., Nota A., Sabbi E., Sirianni M., Clementini G., Costa G. D., Tosi M., Harbeck D., Koch A., Kayser A., Structural Parameters of Seven Small Magellanic Cloud Intermediate-Age and Old Star Clusters, The Astronomical Journal, 2009, vol. 138, p. 1403

Glatt K., Grebel E. K., Sabbi E., Gallagher III J. S., Nota A., Sirianni M., Clementini G., Tosi M., Harbeck D., Koch A., Kayser A., Da Costa G., Age Determination of Six Intermediate-Age Small Magellanic Cloud Star Clusters with HST/ACS, AJ, 2008, vol. 136, p. 1703

González Delgado R. M., Cerviño M., Martins L. P., Leitherer C., Hauschildt P. H., Evolutionary stellar population synthesis at high spectral resolution: optical wavelengths, MNRAS, 2005, vol. 357 , p. 945

Goudfrooij P., Girardi L., Kozhurina-Platais V., Kalirai J. S., Platais I., Puzia T. H., Correnti M., Bressan A., Chandar R., Kerber L., Marigo P., Rubele S., Extended Main Sequence Turnoffs in Intermediate-age Star Clusters: A Correlation between Turnoff Width and Early Escape Velocity, ApJ, 2014, vol. 797, p. 35

Grocholski A. J., Cole A. A., Sarajedini A., Geisler D., Smith V. V., Ca II Triplet Spectroscopy of Large Magellanic Cloud Red Giants. I. Abundances and Velocities for a Sample of Populous Clusters, AJ, 2006, vol. 132, p. 1630

Gupta A. C., CCD Photometric Observations of the Galactic Globular Clusters NGC 1904 and NGC 6341, ArXiv Astrophysics e-prints, 2003

Harris W. E., Globular cluster systems in galaxies beyond the Local Group., ARA\&A, 1991, vol. 29, p. 543

Harris W. E., A Catalog of Parameters for Globular Clusters in the Milky Way, AJ, 1996, vol. 112 , p. 1487

Hill V., Abundances of cool supergiants in the SMC young cluster NGC 330, A\&A, 1999, vol. 345 , p. 430

Johnson C. I., Caldwell N., Rich R. M., Mateo M., Bailey III J. I., Olszewski E. W., Walker M. G., Chemical Complexity in the Eu-enhanced Monometallic Globular NGC 5986, ApJ, 2017, vol. 842, p. 24 
Johnson J. A., Bolte M., Hesser J. E., Ivans I. I., Stetson P. B., Abundances in LMC and SMC Globular Clusters, Origin and Evolution of the Elements, 2004, p. 29

Jones K., Jones K., Messier C., Messier's Nebulae and Star Clusters. Practical Astronomy Handbooks, Cambridge University Press, 1991

Kacharov N., Koch A., McWilliam A., A comprehensive chemical abundance study of the outer halo globular cluster M 75, A\&A, 2013, vol. 554, p. A81

Kennedy E. S., The Observatory in Islam and Its Place in the General History of the Observatory. Aydin Sayili, Isis, 1962, vol. 53, p. 237

Kerber L. O., Santiago B. X., Brocato E., Physical parameters of 15 intermediate-age LMC clusters from modelling of HST colour-magnitude diagrams, A\&A, 2007, vol. 462, p. 139

Khokhlov A., Mueller E., Hoeflich P., Light curves of type IA supernova models with different explosion mechanisms., A\&A, 1993, vol. 270, p. 223

Koleva M., Prugniel P., Bouchard A., Wu Y., ULySS: a full spectrum fitting package, A\&A, 2009, vol. 501, p. 1269

Koleva M., Prugniel P., Ocvirk P., Le Borgne D., Soubiran C., Spectroscopic ages and metallicities of stellar populations: validation of full spectrum fitting, Monthly Notices of the Royal Astronomical Society, 2008, vol. 385, p. 1998

Kormendy J., , 2016 Elliptical Galaxies and Bulges of Disc Galaxies: Summary of Progress and Outstanding Issues. p. 431

Lagioia E. P., Milone A. P., Stetson P. B., Bono G., Prada Moroni P. G., Dall'Ora M., Aparicio A., Buonanno R., Calamida A., Ferraro I., Gilmozzi R., Iannicola G., Matsunaga N., Monelli M., Walker A., On the Kinematic Separation of Field and Cluster Stars across the Bulge Globular NGC 6528, ApJ, 2014, vol. 782, p. 50

Lapenna E., Mucciarelli A., Ferraro F. R., Origlia L., Lanzoni B., Massari D., Dalessandro E., Chemical Analysis of Asymptotic Giant Branch Stars in M62, ApJ, 2015, vol. 813, p. 97 
Larsen S. S., Brodie J. P., Strader J., Detailed abundance analysis from integrated highdispersion spectroscopy: globular clusters in the Fornax dwarf spheroidal, A\&A, 2012, vol. 546, p. A53

Lee J.-W., A Chemical Abundance Study of three RHB and two RGB stars in NGC637 (M69). In Revista Mexicana de Astronomia y Astrofisica Conference Series , vol. 28 of Revista Mexicana de Astronomia y Astrofisica, vol. 27, 2007, p. 120

Lee J.-W., Carney B. W., Chemical Abundances of Three Metal-poor Globular Clusters (NGC 6287, NGC 6293, and NGC 6541) in the Inner Halo, AJ, 2002, vol. 124, p. 1511

Lee J.-W., López-Morales M., Carney B. W., A Chemical Abundance Study of One Red Giant Star in NGC 5694: A Globular Cluster with a Dwarf Spheroidal Chemical Signature?, ApJ, 2006, vol. 646, p. L119

Leitherer C., Schaerer D., Goldader J. D., Delgado R. M. G., Robert C., Kune D. F., de Mello D. F., Devost D., Heckman T. M., Starburst99: Synthesis Models for Galaxies with Active Star Formation, ApJS, 1999, vol. 123, p. 3

Maciel W. J., Introdução à estrutura e evolução estelar. Editora da Universidade de São Paulo, 1999

Mackey A. D., Gilmore G. F., Surface brightness profiles and structural parameters for 53 rich stellar clusters in the Large Magellanic Cloud, MNRAS, 2003, vol. 338, p. 85

Maraston C., Strömbäck G., Stellar population models at high spectral resolution, MNRAS, 2011, vol. 418, p. 2785

Marino A. F., Milone A. P., Karakas A. I., Casagrande L., Yong D., Shingles L., Da Costa G., Norris J. E., Stetson P. B., Lind K., Asplund M., Collet R., Jerjen H., Sbordone L., Aparicio A., Cassisi S., Iron and s-elements abundance variations in NGC 5286: comparison with 'anomalous' globular clusters and Milky Way satellites, MNRAS, 2015, vol. 450 , p. 815

Marino A. F., Milone A. P., Piotto G., Villanova S., Bedin L. R., Bellini A., Renzini A., A double stellar generation in the globular cluster NGC 6656 (M 22). Two stellar groups with different iron and s-process element abundances, A\&A, 2009, vol. 505, p. 1099 
Massari D., Mucciarelli A., Dalessandro E., Bellazzini M., Cassisi S., Fiorentino G., Ibata R. A., Lardo C., Salaris M., The chemical composition of the low-mass Galactic globular cluster NGC 6362, MNRAS, 2017, vol. 468, p. 1249

Mateus A., Sodré L., Cid Fernandes R., Stasińska G., Schoenell W., Gomes J. M., Semiempirical analysis of Sloan Digital Sky Survey galaxies - II. The bimodality of the galaxy population revisited, MNRAS, 2006, vol. 370, p. 721

Matteucci F., What determines galactic evolution?, Ap\&SS, 2003, vol. 284, p. 539

McLaughlin D. E., van der Marel R. P., Resolved Massive Star Clusters in the Milky Way and Its Satellites: Brightness Profiles and a Catalog of Fundamental Parameters, ApJS, 2005a, vol. 161, p. 304

McLaughlin D. E., van der Marel R. P., Resolved Massive Star Clusters in the Milky Way and Its Satellites: Brightness Profiles and a Catalog of Fundamental Parameters, ApJS, 2005b, vol. 161, p. 304

McWilliam A., Abundance Ratios and Galactic Chemical Evolution, ARA\&A, 1997, vol. 35 , p. 503

Meissner F., Weiss A., Global fitting of globular cluster age indicators, A\&A, 2006, vol. 456, p. 1085

Metropolis N., Rosenbluth A. W., Rosenbluth M. N., Teller A. H., Teller E., Equation of State Calculations by Fast Computing Machines, The Journal of Chemical Physics, 1953, vol. 21, p. 1087

Mighell K. J., Sarajedini A., French R. S., WFPC2 Observations of Star Clusters in the Magellanic Clouds. II. The Oldest Star Clusters in the Small Magellanic Cloud, AJ, 1998, vol. 116, p. 2395

Milone A. P., Marino A. F., Dotter A., Norris J. E., Jerjen H., Piotto G., Cassisi S., Bedin L. R., Recio Blanco A., Sarajedini A., Asplund M., Monelli M., Aparicio A., Global and Nonglobal Parameters of Horizontal-branch Morphology of Globular Clusters, ApJ, 2014, vol. 785 , p. 21 
Morgan W. W., The Integrated Spectral Types of Globular Clusters, Publications of the Astronomical Society of the Pacific, 1956, vol. 68, p. 509

Mucciarelli A., Carretta E., Origlia L., Ferraro F. R., The Chemical Composition of Red Giant Stars in Four Intermediate-Age Clusters of the Large Magellanic Cloud, AJ, 2008, vol. 136 , p. 375

Mucciarelli A., Ferraro F. R., Origlia L., Fusi Pecci F., The Globular Cluster NGC 1978 in the Large Magellanic Cloud, AJ, 2007, vol. 133, p. 2053

Mucciarelli A., Origlia L., Ferraro F. R., Chemical Composition of the Old Globular Clusters NGC 1786, NGC 2210, and NGC 2257 in the Large Magellanic Cloud, ApJ, 2010, vol. 717 , p. 277

Mura-Guzmán A., Villanova S., Muñoz C., Tang B., Chemical study of the metal-rich globular cluster NGC 5927, MNRAS, 2018, vol. 474, p. 4541

Niederhofer F., Hilker M., Bastian N., Silva-Villa E., No evidence for significant age spreads in young massive LMC clusters, A\&A, 2015, vol. 575, p. A62

Norris J. E., Ryan S. G., Beers T. C., Extremely Metal-Poor Stars. VIII. High-Resolution, High Signal-to-Noise Ratio Analysis of Five Stars with [Fe/H]\&lt; ₹-3.5, ApJ, 2001, vol. 561, p. 1034

North J., North J., Porter R., The Norton History of Astronomy and Cosmology. Norton history of science, Norton, 1994

Ocvirk P., Fake Star Formation Bursts: Blue Horizontal Branch Stars Masquerade as Young Massive Stars in Optical Integrated Light Spectroscopy, ApJ, 2010, vol. 709, p. 88

Ocvirk P., Pichon C., Lançon A., Thiébaut E., STECKMAP: STEllar Content and Kinematics from high resolution galactic spectra via Maximum A Posteriori, MNRAS, 2006, vol. 365 , p. 74

Olsen K. A. G., Hodge P. W., Mateo M., Olszewski E. W., Schommer R. A., Suntzeff N. B., Walker A. R., HST colour-magnitude diagrams of six old globular clusters in the LMC, Monthly Notices of the Royal Astronomical Society, 1998, vol. 300, p. 665 
Olszewski E. W., Schommer R. A., Suntzeff N. B., Harris H. C., Spectroscopy of giants in LMC clusters. I - Velocities, abundances, and the age-metallicity relation, AJ, 1991, vol. 101 , p. 515

O’Malley E. M., Knaizev A., McWilliam A., Chaboyer B., High-resolution Spectroscopic Abundances of Red Giant Branch Stars in NGC 6681, ApJ, 2017, vol. 846, p. 23

Origlia L., Valenti E., Rich R. M., High-resolution infrared spectra of NGC 6342 and 6528: two moderately reddened bulge globular clusters, MNRAS, 2005, vol. 356, p. 1276

Origlia L., Valenti E., Rich R. M., High resolution infrared spectra of NGC 6440 and NGC 6441: two massive bulge globular clusters, MNRAS, 2008, vol. 388, p. 1419

Pearson K., On the Criterion that a given System of Deviations from the Probable in the Case of a Correlated System of Variables is such that can be reasonably supposed to have arisen from Random Sampling, Philosophical Magazine, 1900, vol. 50, p. 157

Penaloza F., Pessev P., Vasquez S., Borissova J., Kurtev R., Zoccali M., Chemical Abundances of the Highly Obscured Galactic Globular Clusters 2MASS GC02 and Mercer 5, Publications of the Astronomical Society of the Pacific, 2015, vol. 127

Pietrinferni A., Cassisi S., Salaris M., Castelli F., A Large Stellar Evolution Database for Population Synthesis Studies. I. Scaled Solar Models and Isochrones, ApJ, 2004, vol. 612, p. 168

Pietrinferni A., Cassisi S., Salaris M., Castelli F., A Large Stellar Evolution Database for Population Synthesis Studies. II. Stellar Models and Isochrones for an $\alpha$-enhanced Metal Distribution, ApJ, 2006, vol. 642, p. 797

Pipino A., Matteucci F., The timescales of chemical enrichment in the Galaxy. In The Ages of Stars , vol. 258 of IAU Symposium, 2009, p. 39

Prugniel P., Soubiran C., Koleva M., Le Borgne D., VizieR Online Data Catalog: ELODIE library V3.1 (Prugniel+ 2007), VizieR Online Data Catalog, 2007, p. III/251

Renzini A., On the many uses of globular cluster luminosity functions, Mem. Soc. Astron. Italiana, 1986, vol. 57, p. 357 
Rodgers A. W., Harding P., The calcium abundances in NGC 6584, NGC 6864, and NGC 6981, PASP, 1990, vol. 102, p. 235

Roederer I. U., Mateo M., Bailey J. I., Spencer M., Crane J. D., Shectman S. A., Detailed chemical abundances in NGC 5824: another metal-poor globular cluster with internal heavy element abundance variations, MNRAS, 2016, vol. 455, p. 2417

Rojas-Arriagada A., Zoccali M., Vásquez S., Ripepi V., Musella I., Marconi M., Grado A., Limatola L., High resolution spectroscopic analysis of seven giants in the bulge globular cluster NGC 6723, A\&A, 2016, vol. 587, p. A95

Sagan C., Cosmos. Random House, 1980

Sakari C. M., Shetrone M., Venn K., McWilliam A., Dotter A., Spectrum syntheses of high-resolution integrated light spectra of Galactic globular clusters, MNRAS, 2013, vol. 434 , p. 358

Sánchez-Blázquez P., Peletier R. F., Jiménez-Vicente J., Cardiel N., Cenarro A. J., FalcónBarroso J., Gorgas J., Selam S., Vazdekis A., Medium-resolution Isaac Newton Telescope library of empirical spectra, MNRAS, 2006, vol. 371, p. 703

Santos Jr. J. F. C., Piatti A. E., Ages and metallicities of star clusters: New calibrations and diagnostic diagrams from visible integrated spectra, A\&A, 2004, vol. 428, p. 79

Schawinski K., Thomas D., Sarzi M., Maraston C., Kaviraj S., Joo S.-J., Yi S. K., Silk J., Observational evidence for AGN feedback in early-type galaxies, MNRAS, 2007, vol. 382, p. 1415

Schiavon R. P., Population Synthesis in the Blue. IV. Accurate Model Predictions for Lick Indices and UBV Colors in Single Stellar Populations, ApJS, 2007, vol. 171, p. 146

Schiavon R. P., Caldwell N., Rose J. A., The Integrated Spectrum of M67 and the Spectroscopic Age of M32, AJ, 2004, vol. 127, p. 1513

Schiavon R. P., Rose J. A., Courteau S., MacArthur L. A., A Library of Integrated Spectra of Galactic Globular Clusters, ApJS, 2005, vol. 160, p. 163 
Sharma S., Borissova J., Kurtev R., Ivanov V. D., Geisler D., Toward the General Red Giant Branch Slope-Metallicity-Age Calibration. I. Metallicities, Ages, and Kinematics for Eight Large Magellanic Cloud Clusters, AJ, 2010, vol. 139, p. 878

Sirianni M., Nota A., De Marchi G., Leitherer C., Clampin M., The Low End of the Initial Mass Function in Young Clusters. II. Evidence for Primordial Mass Segregation in NGC 330 in the Small Magellanic Cloud, ApJ, 2002, vol. 579, p. 275

Smith B. D., Sigurdsson S., The Transition from the First Stars to the Second Stars in the Early Universe, ApJ, 2007, vol. 661, p. L5

Thomas D., Maraston C., Bender R., Stellar population models of Lick indices with variable element abundance ratios, MNRAS, 2003, vol. 339, p. 897

Thomas D., Maraston C., Bender R., de Oliveira C. M., The Epochs of Early-Type Galaxy Formation as a Function of Environment, ApJ, 2005, vol. 621, p. 673

Till J., Galactic archaeology with metal- poor stars , 2017

Trager S. C., Faber S. M., Worthey G., González J. J., The Stellar Population Histories of Early-Type Galaxies. II. Controlling Parameters of the Stellar Populations, AJ, 2000, vol. 120 , p. 165

Usher C., Pastorello N., Bellstedt S., Alabi A., Cerulo P., Chevalier L., Fraser-McKelvie A., Penny S., Foster C., McDermid R. M., Schiavon R. P., Villaume A., The WAGGS project - I. The WiFeS Atlas of Galactic Globular cluster Spectra, MNRAS, 2017, vol. 468, p. 3828

Valenti E., Origlia L., Rich R. M., High-resolution near-infrared spectra of NGC 6624 and 6569, MNRAS, 2011, vol. 414, p. 2690

Vazdekis A., Evolutionary Stellar Population Synthesis at $2 \AA$ Spectral Resolution, ApJ, 1999, vol. 513, p. 224

Vazdekis A., Casuso E., Peletier R. F., Beckman J. E., A New Chemo-evolutionary Population Synthesis Model for Early-Type Galaxies. I. Theoretical Basis, ApJS, 1996, vol. 106, p. 307 
Vazdekis A., Cenarro A. J., Gorgas J., Cardiel N., Peletier R. F., Empirical calibration of the near-infrared CaII triplet - IV. The stellar population synthesis models, MNRAS, 2003, vol. 340, p. 1317

Vazdekis A., Coelho P., Cassisi S., Ricciardelli E., Falcón-Barroso J., Sánchez-Blázquez P., Barbera F. L., Beasley M. A., Pietrinferni A., Evolutionary stellar population synthesis with MILES - II. Scaled-solar and alpha-enhanced models, MNRAS, 2015, vol. 449, p. 1177

Vazdekis A., Sánchez-Blázquez P., Falcón-Barroso J., Cenarro A. J., Beasley M. A., Cardiel N., Gorgas J., Peletier R. F., Evolutionary stellar population synthesis with MILES - I. The base models and a new line index system, MNRAS, 2010, vol. 404, p. 1639

Villanova S., Monaco L., Moni Bidin C., Assmann P., A spectroscopic study of the globular Cluster NGC 4147, MNRAS, 2016, vol. 460, p. 2351

Villanova S., Piotto G., King I. R., Anderson J., Bedin L. R., Gratton R. G., Cassisi S., Momany Y., Bellini A., Cool A. M., Recio-Blanco A., Renzini A., The Multiplicity of the Subgiant Branch of omega Centauri: Evidence for Prolonged Star Formation, ApJ, 2007, vol. 663, p. 296

Walcher C. J., Böker T., Charlot S., Ho L. C., Rix H. W., Rossa J., Shields J. C., van der Marel R. P., Stellar Populations in the Nuclei of Late-Type Spiral Galaxies, ApJ, 2006, vol. 649 , p. 692

Walcher C. J., Coelho P., Gallazzi A., Charlot S., Differential stellar population models: how to reliably measure $[\mathrm{Fe} / \mathrm{H}]$ and $[\mathrm{alpha} / \mathrm{Fe}]$ in galaxies, MNRAS, 2009, vol. 398, p. L44

Walcher J., Groves B., Budavári T., Dale D., Fitting the integrated spectral energy distributions of galaxies, Ap\&SS, 2011, vol. 331, p. 1

Wood D. B., Multicolor Photoelectric Photometry of Galaxies, ApJ, 1966, vol. 145, p. 36

Worthey G., Comprehensive Stellar Population Models and the Disentanglement of Age and Metallicity Effects, ApJS, 1994, vol. 95, p. 107 
Zhao F.-Y., Strom R. G., Jiang S.-Y., The Guest Star of AD185 must have been a Supernova, Chinese Journal of Astronomy and Astrophysics, 2006, vol. 6, p. 635

Zinn R., West M. J., The globular cluster system of the Galaxy. III. Measurements of radial velocity and metallicity for 60 clusters and a compilation of metallicities for 121 clusters., ApJS, 1984, vol. 55, p. 45

Zoccali M., Renzini A., Ortolani S., Bica E., Barbuy B., The Proper Motion of the Globular Cluster NGC 6553 and of Bulge Stars with the Hubble Space Telescope, The Astronomical Journal, 2001, vol. 121, p. 2638 
Apêndice 



\section{Apêndice A}

\section{Velocidades radiais e valores de sinal-ruído}

Tabela A.1 - Velocidades radiais (calculadas como descrito na seção 3.3 e razão sinal-ruído médio para cada espectro da biblioteca WAGGS.

\begin{tabular}{lrr}
\hline Arquivo & Velocidade radial $(\mathrm{km} / \mathrm{s})$ & $\langle\mathrm{SNR}\rangle$ \\
\hline Fornax3_B7000_2015-08-09 & $61.2 \pm 2.0$ & 40 \\
Fornax4_B7000_2015-08-10 & $50.7 \pm 0.5$ & 20 \\
Fornax5_B7000_2015-08-11 & $62.0 \pm 1.6$ & 13 \\
NGC0104_B7000_2015-01-30 & $-17.5 \pm 0.5$ & 359 \\
NGC0121_B7000_2015-08-11 & $143.5 \pm 0.5$ & 57 \\
NGC0330_B7000_2015-11-02 & $155.0 \pm 4.4$ & 24 \\
NGC0361_B7000_2015-11-02 & $173.8 \pm 0.9$ & 13 \\
NGC0362_B7000_2015-01-30 & $223.5 \pm 0.4$ & 176 \\
NGC0416_B7000_2015-08-08 & $157.4 \pm 0.6$ & 32 \\
NGC0419_B7000_2015-12-18 & $185.9 \pm 1.1$ & 44 \\
NGC1261_B7000_2015-08-08 & $73.4 \pm 0.7$ & 78 \\
NGC1783_B7000_2016-04-02 & $277.5 \pm 0.8$ & 27 \\
NGC1786_B7000_2016-10-03 & $257.8 \pm 1.6$ & 25 \\
NGC1846_B7000_2016-04-01 & $240.1 \pm 1.6$ & 2 \\
NGC1846_B7000_2016-04-02 & $240.7 \pm 0.7$ & 8 \\
NGC1850_B7000_2016-04-01 & $248.2 \pm 4.2$ & 37 \\
NGC1851_B7000_2015-01-31 & $322.4 \pm 0.5$ & 262 \\
NGC1856_B7000_2016-04-02 & $265.8 \pm 3.9$ & 37 \\
NGC1866_B7000_2016-04-02 & $301.5 \pm 3.0$ & 38 \\
NGC1868_B7000_2016-10-03 & $285.4 \pm 3.0$ & 19 \\
\hline
\end{tabular}




\begin{tabular}{|c|c|c|}
\hline Arquivo & Velocidade radial $(\mathrm{km} / \mathrm{s})$ & $\langle\mathrm{SNR}\rangle$ \\
\hline NGC1898_B7000_2016-10-03 & $209.0 \pm 0.8$ & 11 \\
\hline NGC1904_B7000_2015-01-30 & $205.4 \pm 0.9$ & 68 \\
\hline NGC1916_B7000_2015-12-18 & $272.6 \pm 1.4$ & 45 \\
\hline NGC1978_B7000_2016-10-03 & $290.1 \pm 0.8$ & 25 \\
\hline NGC2004_B7000_2016-09-30 & $294.6 \pm 2.8$ & 11 \\
\hline NGC2019_B7000_2016-09-30 & $265.8 \pm 1.0$ & 19 \\
\hline NGC2100_B7000_2015-12-17 & $33.9 \pm 4.3$ & 21 \\
\hline NGC2136_B7000_2016-10-03 & $269.1 \pm 3.9$ & 24 \\
\hline NGC2808_B7000_2015-01-31 & $106.0 \pm 0.4$ & 271 \\
\hline NGC2808_B7000_2015-07-10 & $104.8 \pm 0.4$ & 194 \\
\hline NGC3201_B7000_2015-04-15 & $493.9 \pm 0.5$ & 76 \\
\hline NGC4147_B7000_2015-01-30 & $182.5 \pm 1.5$ & 17 \\
\hline NGC4590_B7000_2015-04-13 & $-90.1 \pm 2.0$ & 73 \\
\hline NGC4833_B7000_2015-01-30 & $203.1 \pm 2.0$ & 73 \\
\hline NGC5024_B7000_2015-01-30 & $-63.4 \pm 1.2$ & 60 \\
\hline NGC5139_B7000_2015-04-13 & $231.4 \pm 1.2$ & 45 \\
\hline NGC5272_B7000_2016-04-02 & $-145.0 \pm 0.8$ & 93 \\
\hline NGC5286_B7000_2015-04-14 & $61.8 \pm 0.9$ & 75 \\
\hline NGC5634_B7000_2015-08-10 & $-17.9 \pm 1.3$ & 83 \\
\hline NGC5694_B7000_2015-08-11 & $-142.9 \pm 1.3$ & 107 \\
\hline NGC5824_B7000_2016-04-01 & $-25.5 \pm 1.3$ & 43 \\
\hline NGC5904_B7000_2015-04-15 & $58.0 \pm 0.7$ & 119 \\
\hline NGC5927_B7000_2015-01-31 & $-105.0 \pm 0.6$ & 43 \\
\hline NGC5986_B7000_2015-07-10 & $96.4 \pm 0.6$ & 61 \\
\hline NGC6093_B7000_2015-04-14 & $15.0 \pm 0.9$ & 13 \\
\hline NGC6121_B7000_2015-04-15 & $75.6 \pm 0.6$ & 36 \\
\hline NGC6139_B7000_2015-08-11 & $21.4 \pm 0.5$ & 34 \\
\hline NGC6171_B7000_2015-07-09 & $-35.5 \pm 0.4$ & 74 \\
\hline NGC6218_B7000_2015-07-09 & $-48.2 \pm 0.5$ & 53 \\
\hline NGC6254_B7000_2015-04-13 & $78.2 \pm 0.6$ & 49 \\
\hline NGC6266_B7000_2015-08-09 & $-74.1 \pm 0.4$ & 105 \\
\hline
\end{tabular}




\begin{tabular}{|c|c|c|}
\hline Arquivo & Velocidade radial $(\mathrm{km} / \mathrm{s})$ & $\langle\mathrm{SNR}\rangle$ \\
\hline NGC6273_B7000_2015-08-10 & $142.7 \pm 0.7$ & 72 \\
\hline NGC6284_B7000_2016-04-01 & $29.5 \pm 0.4$ & 29 \\
\hline NGC6284_B7000_2016-09-29 & $36.0 \pm 0.9$ & 24 \\
\hline NGC6293_B7000_2015-08-11 & $-141.0 \pm 1.4$ & 72 \\
\hline NGC6304_B7000_2015-04-14 & $-109.1 \pm 0.6$ & 13 \\
\hline NGC6304_B7000_2015-08-08 & $-105.4 \pm 0.5$ & 16 \\
\hline NGC6316_B7000_2016-04-02 & $98.4 \pm 0.6$ & 41 \\
\hline NGC6333_B7000_2016-04-02 & $315.3 \pm 0.7$ & 68 \\
\hline NGC6342_B7000_2015-07-10 & $118.6 \pm 0.5$ & 17 \\
\hline NGC6342_B7000_2015-08-10 & $119.5 \pm 0.5$ & 13 \\
\hline NGC6352_B7000_2015-07-09 & $-127.6 \pm 0.5$ & 27 \\
\hline NGC6356_B7000_2016-04-01 & $49.2 \pm 0.5$ & 40 \\
\hline NGC6362_B7000_2015-08-11 & $-10.0 \pm 0.8$ & 67 \\
\hline NGC6388_B7000_2015-04-13 & $83.1 \pm 0.5$ & 175 \\
\hline NGC6397_B7000_2015-04-13 & $18.3 \pm 3.5$ & 81 \\
\hline NGC6440_B7000_2016-10-03 & $-71.0 \pm 1.4$ & 11 \\
\hline NGC6441_B7000_2015-04-15 & $22.3 \pm 0.6$ & 120 \\
\hline NGC6522_B7000_2016-04-02 & $-9.9 \pm 0.5$ & 75 \\
\hline NGC6528_B7000_2015-04-13 & $211.7 \pm 0.6$ & 12 \\
\hline NGC6541_B7000_2015-04-14 & $-163.8 \pm 1.0$ & 134 \\
\hline NGC6553_B7000_2015-04-15 & $1.7 \pm 0.8$ & 14 \\
\hline NGC6569_B7000_2015-08-10 & $-52.1 \pm 0.5$ & 35 \\
\hline NGC6584_B7000_2015-08-08 & $262.4 \pm 0.6$ & 22 \\
\hline NGC6624_B7000_2015-07-09 & $52.8 \pm 0.4$ & 67 \\
\hline NGC6637_B7000_2015-07-09 & $46.6 \pm 0.5$ & 67 \\
\hline NGC6652_B7000_2015-08-08 & $-97.3 \pm 0.5$ & 27 \\
\hline NGC6656_B7000_2015-07-09 & $-142.8 \pm 0.7$ & 64 \\
\hline NGC6681_B7000_2015-08-10 & $218.3 \pm 0.9$ & 56 \\
\hline NGC6715_B7000_2015-07-09 & $144.4 \pm 0.6$ & 72 \\
\hline NGC6717_B7000_2015-04-15 & $31.0 \pm 0.5$ & 24 \\
\hline NGC6717_B7000_2016-04-01 & $31.0 \pm 0.6$ & 41 \\
\hline
\end{tabular}




\begin{tabular}{|c|c|c|}
\hline Arquivo & Velocidade radial $(\mathrm{km} / \mathrm{s})$ & $\langle\mathrm{SNR}\rangle$ \\
\hline NGC6723_B7000_2016-09-29 & $-98.2 \pm 0.7$ & 40 \\
\hline NGC6752_B7000_2015-04-14 & $-24.7 \pm 1.0$ & 111 \\
\hline NGC6809_B7000_2015-08-09 & $180.3 \pm 1.9$ & 114 \\
\hline NGC6838_B7000_2015-08-08 & $-29.9 \pm 0.6$ & 39 \\
\hline NGC6864_B7000_2015-08-10 & $-187.2 \pm 0.4$ & 64 \\
\hline NGC6934_B7000_2015-08-08 & $-405.5 \pm 1.0$ & 19 \\
\hline NGC7006_B7000_2015-08-09 & $-380.3 \pm 0.9$ & 31 \\
\hline NGC7078_B7000_2015-07-09 & $-108.3 \pm 2.0$ & 105 \\
\hline NGC7089_B7000_2015-07-09 & $-3.3 \pm 1.0$ & 147 \\
\hline NGC7099_B7000_2015-07-09 & $-180.4 \pm 2.9$ & 16 \\
\hline NGC7099_B7000_2016-09-30 & $-190.1 \pm 3.7$ & 33 \\
\hline NGC7099_B7000_2016-10-01 & $-189.4 \pm 4.5$ & 66 \\
\hline Fornax4_R7000_2015-08-10 & $46.6 \pm 0.5$ & 32 \\
\hline Fornax5_R7000_2015-08-11 & $71.9 \pm 2.0$ & 12 \\
\hline NGC0104_R7000_2015-01-30 & $-15.9 \pm 0.7$ & 727 \\
\hline NGC0121_R7000_2015-08-11 & $144.9 \pm 0.5$ & 97 \\
\hline NGC0330_R7000_2015-11-02 & $154.9 \pm 1.1$ & 61 \\
\hline NGC0361_R7000_2015-11-02 & $168.7 \pm 3.0$ & 34 \\
\hline NGC0362_R7000_2015-01-30 & $222.1 \pm 0.7$ & 306 \\
\hline NGC0416_R7000_2015-08-08 & $155.9 \pm 0.6$ & 55 \\
\hline NGC0419_R7000_2015-12-18 & $201.1 \pm 0.8$ & 71 \\
\hline NGC1261_R7000_2015-08-08 & $71.4 \pm 0.4$ & 149 \\
\hline NGC1783_R7000_2016-04-02 & $279.2 \pm 0.8$ & 43 \\
\hline NGC1786_R7000_2016-10-03 & $259.1 \pm 1.1$ & 42 \\
\hline NGC1846_R7000_2016-04-02 & $241.3 \pm 0.8$ & 17 \\
\hline NGC1850_R7000_2016-04-01 & $244.6 \pm 3.8$ & 66 \\
\hline NGC1851_R7000_2015-01-31 & $321.9 \pm 0.6$ & 379 \\
\hline NGC1856_R7000_2016-04-02 & $265.4 \pm 1.2$ & 58 \\
\hline NGC1866_R7000_2016-04-02 & $301.8 \pm 0.9$ & 47 \\
\hline NGC1868_R7000_2016-10-03 & $284.6 \pm 1.3$ & 22 \\
\hline NGC1898_R7000_2016-10-03 & $208.1 \pm 1.0$ & 20 \\
\hline
\end{tabular}




\begin{tabular}{|c|c|c|}
\hline Arquivo & Velocidade radial $(\mathrm{km} / \mathrm{s})$ & $\langle\mathrm{SNR}\rangle$ \\
\hline NGC1904_R7000_2015-01-30 & $204.2 \pm 0.6$ & 109 \\
\hline NGC1916_R7000_2015-12-18 & $297.8 \pm 1.1$ & 71 \\
\hline NGC1978_R7000_2016-10-03 & $292.0 \pm 0.8$ & 24 \\
\hline NGC2004_R7000_2016-09-30 & $307.4 \pm 5.8$ & 1 \\
\hline NGC2019_R7000_2016-09-30 & $277.1 \pm 1.5$ & 11 \\
\hline NGC2100_R7000_2015-12-17 & $253.4 \pm 2.3$ & 24 \\
\hline NGC2136_R7000_2016-10-03 & $269.9 \pm 1.1$ & 23 \\
\hline NGC2808_R7000_2015-01-31 & $107.3 \pm 0.8$ & 474 \\
\hline NGC2808_R7000_2015-07-09 & $104.4 \pm 0.8$ & 244 \\
\hline NGC3201_R7000_2015-01-31 & $496.9 \pm 0.7$ & 163 \\
\hline NGC3201_R7000_2015-04-15 & $496.8 \pm 0.9$ & 188 \\
\hline NGC4147_R7000_2015-01-30 & $181.8 \pm 1.0$ & 32 \\
\hline NGC4590_R7000_2015-04-13 & $-96.9 \pm 3.2$ & 176 \\
\hline NGC4833_R7000_2015-01-30 & $203.0 \pm 1.7$ & 90 \\
\hline NGC5024_R7000_2015-01-30 & $-63.1 \pm 0.9$ & 102 \\
\hline NGC5139_R7000_2015-04-13 & $233.9 \pm 1.7$ & 63 \\
\hline NGC5272_R7000_2016-04-02 & $-142.6 \pm 0.8$ & 133 \\
\hline NGC5286_R7000_2015-04-14 & $62.3 \pm 1.5$ & 180 \\
\hline NGC5286_R7000_2016-04-01 & $59.8 \pm 1.3$ & 64 \\
\hline NGC5634_R7000_2015-08-10 & $-14.0 \pm 0.8$ & 158 \\
\hline NGC5694_R7000_2015-08-11 & $-137.5 \pm 1.0$ & 169 \\
\hline NGC5824_R7000_2016-04-01 & $-22.0 \pm 1.3$ & 19 \\
\hline NGC5904_R7000_2015-04-15 & $57.7 \pm 0.8$ & 189 \\
\hline NGC5927_R7000_2015-01-31 & $-105.4 \pm 1.0$ & 117 \\
\hline NGC5986_R7000_2015-07-10 & $97.1 \pm 1.3$ & 116 \\
\hline NGC6093_R7000_2015-04-14 & $10.9 \pm 1.0$ & 60 \\
\hline NGC6121_R7000_2015-04-15 & $74.9 \pm 1.0$ & 76 \\
\hline NGC6139_R7000_2015-08-11 & $22.3 \pm 1.3$ & 111 \\
\hline NGC6171_R7000_2015-07-09 & $-31.6 \pm 0.7$ & 134 \\
\hline NGC6218_R7000_2015-07-09 & $-40.9 \pm 0.6$ & 102 \\
\hline NGC6254_R7000_2015-04-13 & $70.5 \pm 0.9$ & 125 \\
\hline
\end{tabular}




\begin{tabular}{|c|c|c|}
\hline Arquivo & Velocidade radial $(\mathrm{km} / \mathrm{s})$ & $\langle\mathrm{SNR}\rangle$ \\
\hline NGC6266_R7000_2015-08-09 & $-73.4 \pm 1.2$ & 298 \\
\hline NGC6273_R7000_2015-08-10 & $143.2 \pm 1.0$ & 159 \\
\hline NGC6284_R7000_2016-09-29 & $22.7 \pm 0.8$ & 67 \\
\hline NGC6293_R7000_2015-08-11 & $-141.9 \pm 2.0$ & 142 \\
\hline NGC6304_R7000_2015-04-14 & $-108.2 \pm 0.9$ & 65 \\
\hline NGC6304_R7000_2015-08-08 & $-100.2 \pm 0.8$ & 85 \\
\hline NGC6316_R7000_2016-04-02 & $100.4 \pm 1.0$ & 117 \\
\hline NGC6333_R7000_2016-04-02 & $313.9 \pm 0.9$ & 111 \\
\hline NGC6342_R7000_2015-08-10 & $120.6 \pm 0.7$ & 133 \\
\hline NGC6352_R7000_2015-07-09 & $-125.2 \pm 0.9$ & 60 \\
\hline NGC6356_R7000_2016-04-01 & $47.4 \pm 0.7$ & 89 \\
\hline NGC6362_R7000_2015-08-11 & $-8.4 \pm 0.9$ & 143 \\
\hline NGC6388_R7000_2015-04-13 & $80.8 \pm 1.2$ & 281 \\
\hline NGC6397_R7000_2015-04-13 & $12.2 \pm 2.1$ & 111 \\
\hline NGC6440_R7000_2016-10-03 & $-61.2 \pm 1.2$ & 68 \\
\hline NGC6441_R7000_2015-04-15 & $10.0 \pm 1.0$ & 285 \\
\hline NGC6522_R7000_2016-04-02 & $-10.1 \pm 0.9$ & 172 \\
\hline NGC6528_R7000_2015-04-13 & $210.5 \pm 0.8$ & 34 \\
\hline NGC6541_R7000_2015-04-14 & $-163.4 \pm 1.3$ & 245 \\
\hline NGC6553_R7000_2015-04-15 & $2.2 \pm 0.9$ & 61 \\
\hline NGC6569_R7000_2015-08-10 & $-45.8 \pm 0.8$ & 92 \\
\hline NGC6584_R7000_2015-08-08 & $259.2 \pm 0.7$ & 69 \\
\hline NGC6624_R7000_2015-07-09 & $50.7 \pm 0.8$ & 142 \\
\hline NGC6637_R7000_2015-07-09 & $43.4 \pm 0.9$ & 131 \\
\hline NGC6652_R7000_2015-08-08 & $-91.0 \pm 0.6$ & 90 \\
\hline NGC6656_R7000_2015-07-09 & $-142.8 \pm 1.2$ & 137 \\
\hline NGC6681_R7000_2015-08-10 & $219.9 \pm 0.8$ & 104 \\
\hline NGC6715_R7000_2015-07-09 & $142.8 \pm 0.7$ & 233 \\
\hline NGC6717_R7000_2016-04-01 & $26.6 \pm 0.7$ & 107 \\
\hline NGC6723_R7000_2015-07-10 & $-96.2 \pm 0.7$ & 138 \\
\hline NGC6723_R7000_2016-04-01 & $-95.9 \pm 0.6$ & 47 \\
\hline
\end{tabular}




\begin{tabular}{|c|c|c|}
\hline Arquivo & Velocidade radial $(\mathrm{km} / \mathrm{s})$ & $\langle\mathrm{SNR}\rangle$ \\
\hline NGC6723_R7000_2016-09-29 & $-95.4 \pm 0.6$ & 75 \\
\hline NGC6752_R7000_2015-04-14 & $-26.3 \pm 0.9$ & 91 \\
\hline NGC6809_R7000_2015-08-09 & $171.8 \pm 1.3$ & 175 \\
\hline NGC6838_R7000_2015-08-08 & $-24.2 \pm 0.7$ & 192 \\
\hline NGC6864_R7000_2015-08-10 & $-188.7 \pm 0.7$ & 144 \\
\hline NGC6934_R7000_2015-08-08 & $-407.6 \pm 1.0$ & 89 \\
\hline NGC7006_R7000_2015-08-09 & $-383.3 \pm 1.1$ & 138 \\
\hline NGC7078_R7000_2015-07-09 & $-101.8 \pm 2.1$ & 323 \\
\hline NGC7089_R7000_2015-07-09 & $-3.5 \pm 0.7$ & 240 \\
\hline NGC7099_R7000_2015-07-09 & $-182.0 \pm 2.7$ & 27 \\
\hline NGC7099_R7000_2016-09-30 & $-187.2 \pm 1.8$ & 135 \\
\hline NGC7099_R7000_2016-10-01 & $-186.1 \pm 1.8$ & 102 \\
\hline Fornax3_U7000_2015-08-09 & $64.2 \pm 3.4$ & 9 \\
\hline Fornax4_U7000_2015-08-10 & $45.6 \pm 7.0$ & 4 \\
\hline Fornax5_U7000_2015-08-11 & - & 2 \\
\hline NGC0104_U7000_2015-01-30 & $-14.8 \pm 2.3$ & 74 \\
\hline NGC0121_U7000_2015-08-11 & $145.5 \pm 2.6$ & 10 \\
\hline NGC0330_U7000_2015-11-02 & $164.2 \pm 11.7$ & 16 \\
\hline NGC0361_U7000_2015-11-02 & $171.1 \pm 14.1$ & 4 \\
\hline NGC0362_U7000_2015-01-30 & $223.0 \pm 2.2$ & 28 \\
\hline NGC0416_U7000_2015-08-08 & $156.7 \pm 3.4$ & 6 \\
\hline NGC0419_U7000_2015-12-18 & $196.9 \pm 4.9$ & 8 \\
\hline NGC1261_U7000_2015-08-08 & $73.1 \pm 2.8$ & 19 \\
\hline NGC1783_U7000_2016-04-02 & $279.2 \pm 5.2$ & 5 \\
\hline NGC1786_U7000_2016-10-03 & $258.8 \pm 5.8$ & 5 \\
\hline NGC1846_U7000_2016-04-02 & - & 1 \\
\hline NGC1850_U7000_2016-04-01 & $255.9 \pm 12.0$ & 16 \\
\hline NGC1851_U7000_2015-01-31 & $321.4 \pm 2.6$ & 45 \\
\hline NGC1856_U7000_2016-04-02 & - & 12 \\
\hline NGC1866_U7000_2016-04-02 & $306.5 \pm 8.1$ & 9 \\
\hline NGC1868_U7000_2016-10-03 & $277.6 \pm 9.1$ & 3 \\
\hline
\end{tabular}




\begin{tabular}{|c|c|c|}
\hline Arquivo & Velocidade radial $(\mathrm{km} / \mathrm{s})$ & $\langle\mathrm{SNR}\rangle$ \\
\hline NGC1898_U7000_2016-10-03 & $211.4 \pm 7.9$ & 2 \\
\hline NGC1904_U7000_2015-01-30 & $205.6 \pm 2.5$ & 13 \\
\hline NGC1916_U7000_2015-12-18 & $284.8 \pm 7.0$ & 8 \\
\hline NGC1978_U7000_2016-10-03 & $284.4 \pm 10.2$ & 2 \\
\hline NGC2004_U7000_2016-09-30 & - & 0 \\
\hline NGC2019_U7000_2016-09-30 & - & 1 \\
\hline NGC2100_U7000_2015-12-17 & - & 11 \\
\hline NGC2136_U7000_2016-10-03 & $267.1 \pm 10.1$ & 4 \\
\hline NGC2808_U7000_2015-01-31 & $106.0 \pm 2.8$ & 45 \\
\hline NGC2808_U7000_2015-07-09 & $102.7 \pm 2.8$ & 19 \\
\hline NGC3201_U7000_2015-01-31 & $497.7 \pm 2.3$ & 11 \\
\hline NGC3201_U7000_2015-04-15 & $500.7 \pm 3.2$ & 14 \\
\hline NGC4147_U7000_2015-01-30 & $184.0 \pm 14.2$ & 4 \\
\hline NGC4590_U7000_2015-04-13 & $-98.1 \pm 8.7$ & 29 \\
\hline NGC4833_U7000_2015-01-30 & $200.6 \pm 5.7$ & 8 \\
\hline NGC5024_U7000_2015-01-30 & $-62.9 \pm 3.6$ & 11 \\
\hline NGC5139_U7000_2015-04-13 & $234.0 \pm 10.1$ & 6 \\
\hline NGC5272_U7000_2016-04-02 & $-144.4 \pm 2.6$ & 6 \\
\hline NGC5286_U7000_2015-04-14 & $64.6 \pm 3.5$ & 17 \\
\hline NGC5286_U7000_2016-04-01 & $60.9 \pm 2.3$ & 6 \\
\hline NGC5634_U7000_2015-08-10 & $-15.1 \pm 3.3$ & 20 \\
\hline NGC5694_U7000_2015-08-11 & $-141.5 \pm 3.2$ & 20 \\
\hline NGC5824_U7000_2016-04-01 & $-26.6 \pm 10.4$ & 2 \\
\hline NGC5904_U7000_2015-04-15 & $56.3 \pm 3.0$ & 23 \\
\hline NGC5927_U7000_2015-01-31 & $-104.8 \pm 3.9$ & 4 \\
\hline NGC5986_U7000_2015-07-10 & $97.6 \pm 3.0$ & 10 \\
\hline NGC6093_U7000_2015-04-14 & $14.3 \pm 5.9$ & 6 \\
\hline NGC6121_U7000_2015-04-15 & $73.2 \pm 5.3$ & 6 \\
\hline NGC6139_U7000_2015-08-11 & $23.5 \pm 14.1$ & 4 \\
\hline NGC6171_U7000_2015-07-09 & $-39.8 \pm 2.1$ & 8 \\
\hline NGC6218_U7000_2015-07-09 & $-42.1 \pm 2.6$ & 8 \\
\hline
\end{tabular}




\begin{tabular}{|c|c|c|}
\hline Arquivo & Velocidade radial $(\mathrm{km} / \mathrm{s})$ & $\langle\mathrm{SNR}\rangle$ \\
\hline NGC6254_U7000_2015-04-13 & $65.1 \pm 4.4$ & 11 \\
\hline NGC6266_U7000_2015-08-09 & $-77.4 \pm 3.4$ & 19 \\
\hline NGC6273_U7000_2015-08-10 & $142.5 \pm 3.3$ & 10 \\
\hline NGC6284_U7000_2016-09-29 & $25.4 \pm 7.4$ & 4 \\
\hline NGC6293_U7000_2015-08-11 & $-142.2 \pm 4.5$ & 11 \\
\hline NGC6304_U7000_2015-04-14 & $-109.3 \pm 6.8$ & 3 \\
\hline NGC6304_U7000_2015-08-08 & $-97.9 \pm 4.0$ & 4 \\
\hline NGC6316_U7000_2016-04-02 & $99.7 \pm 4.1$ & 4 \\
\hline NGC6333_U7000_2016-04-02 & $315.6 \pm 3.3$ & 7 \\
\hline NGC6342_U7000_2015-08-10 & $122.7 \pm 4.1$ & 6 \\
\hline NGC6352_U7000_2015-07-09 & $-124.9 \pm 3.8$ & 3 \\
\hline NGC6356_U7000_2016-04-01 & $49.6 \pm 1.9$ & 5 \\
\hline NGC6362_U7000_2015-08-11 & $-11.6 \pm 2.2$ & 10 \\
\hline NGC6388_U7000_2015-04-13 & $81.6 \pm 3.2$ & 17 \\
\hline NGC6397_U7000_2015-04-13 & $22.9 \pm 5.1$ & 18 \\
\hline NGC6440_U7000_2016-10-03 & - & 1 \\
\hline NGC6441_U7000_2015-04-15 & $14.8 \pm 3.2$ & 15 \\
\hline NGC6522_U7000_2016-04-02 & $-9.6 \pm 2.9$ & 8 \\
\hline NGC6528_U7000_2015-04-13 & - & 1 \\
\hline NGC6541_U7000_2015-04-14 & $-163.9 \pm 3.6$ & 31 \\
\hline NGC6553_U7000_2015-04-15 & - & 1 \\
\hline NGC6569_U7000_2015-08-10 & $-46.3 \pm 11.5$ & 3 \\
\hline NGC6584_U7000_2015-08-08 & $260.7 \pm 2.5$ & 6 \\
\hline NGC6624_U7000_2015-07-09 & $52.7 \pm 1.9$ & 8 \\
\hline NGC6637_U7000_2015-07-09 & $43.9 \pm 1.8$ & 9 \\
\hline NGC6652_U7000_2015-08-08 & $-96.3 \pm 2.0$ & 7 \\
\hline NGC6656_U7000_2015-07-09 & $-144.8 \pm 3.6$ & 10 \\
\hline NGC6681_U7000_2015-08-10 & $217.6 \pm 4.5$ & 11 \\
\hline NGC6715_U7000_2015-07-09 & $142.1 \pm 3.3$ & 25 \\
\hline NGC6717_U7000_2016-04-01 & $28.4 \pm 2.2$ & 8 \\
\hline NGC6723_U7000_2015-07-10 & $-97.9 \pm 2.3$ & 13 \\
\hline
\end{tabular}




\begin{tabular}{lrr}
\hline Arquivo & Velocidade radial $(\mathrm{km} / \mathrm{s})$ & $\langle\mathrm{SNR}\rangle$ \\
\hline NGC6723_U7000_2016-04-01 & $-97.7 \pm 3.1$ & 4 \\
NGC6723_U7000_2016-09-29 & $-98.4 \pm 4.0$ & 7 \\
NGC6752_U7000_2015-04-14 & $-27.2 \pm 3.2$ & 11 \\
NGC6809_U7000_2015-08-09 & $168.3 \pm 3.3$ & 27 \\
NGC6838_U7000_2015-08-08 & $-19.3 \pm 2.0$ & 10 \\
NGC6864_U7000_2015-08-10 & $-191.0 \pm 2.7$ & 11 \\
NGC6934_U7000_2015-08-08 & $-404.6 \pm 5.5$ & 6 \\
NGC7006_U7000_2015-08-09 & $-382.8 \pm 3.2$ & 14 \\
NGC7078_U7000_2015-07-09 & $-104.1 \pm 4.8$ & 43 \\
NGC7089_U7000_2015-07-09 & $-4.1 \pm 3.5$ & 33 \\
NGC7099_U7000_2015-07-09 & $-187.1 \pm 9.0$ & 4 \\
NGC7099_U7000_2016-09-30 & $-187.7 \pm 5.6$ & 21 \\
NGC7099_U7000_2016-10-01 & $-189.6 \pm 5.1$ & 15 \\
\hline
\end{tabular}




\title{
Apêndice B
}

\section{Script usado na convolução dos espectros}

\author{
from math import * \\ import numpy as $\mathrm{np}$ \\ import scipy.interpolate as $\mathrm{s}$ \\ import scipy.signal as ss \\ from scipy import arange, array, exp \\ from scipy.ndimage import gaussian_filter
}

\#Python version of convolve_rtofwhm.pro by pcoelho

\#Writen: Mar 18th 2019

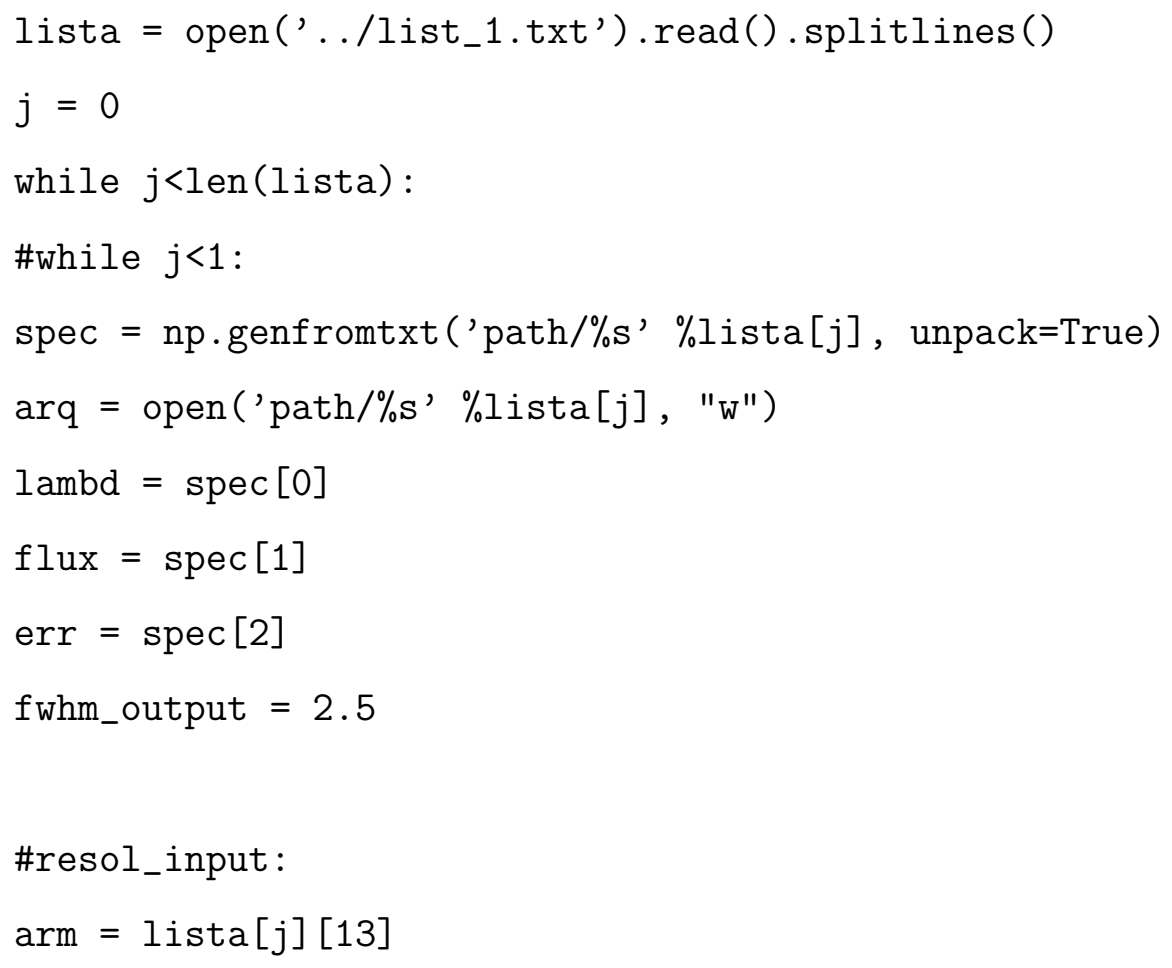


if $\operatorname{arm}==$ ' $U$ ':

$\mathrm{a}=-3.14065518 \mathrm{e}-03$

$b=2.70772425 e+01$

$c=-5.09265633 e+04$

elif $\operatorname{arm}==$ 'B':

$a=7.49796944 e-04$

$b=-5.92841923 e+00$

$c=1.73738613 e+04$

elif arm $==$ ' $R$ ':

$\mathrm{a}=1.38747364 \mathrm{e}-04$

$b=-1.93845959 e-01$

$c=2.74935679 \mathrm{e}+03$

elif arm=='I':

$\mathrm{a}=-2.03974244 \mathrm{e}-07$

$b=8.13850797 e-01$

$c=4.10352225 \mathrm{e}+02$

resol_input $=\mathrm{a} *(\mathrm{lambd}) * * 2+\mathrm{b} * 1 \mathrm{ambd}+\mathrm{c}$

pix_bin $=1 \operatorname{ambd}[1]-\operatorname{lambd}[0]$

filter_width $=10$ \#pixels

extrap_lambda $=n p$. arange $(\operatorname{lambd}[0]-10 *$ pix_bin, lambd[-1]+10*pix_bin, pix_bin $)$

interp_function = s.interpld(lambd, flux, fill_value="extrapolate")

inter_flux = interp_function(extrap_lambda)

interp_function2 = s.interp1d(lambd, err, fill_value="extrapolate")

inter_err = interp_function2(extrap_lambda)

fwhm_input $=$ lambd/resol_input

fwhm_input_pix $=$ fwhm_input $/$ pix_bin

fwhm_output_pix $=$ fwhm_output $/$ pix_bin 
$i=10$

while $i<($ len (lambd)):

fwhm_convol $=\operatorname{sqrt}(f$ whm_output_pix**2 - fwhm_input_pix[i]**2)

if $\mathrm{fwhm}_{-}$convol $<0$ :

print $\left(r^{\prime} \$ \backslash\right.$ lambda $\$=\%$ : something is wrong and the FWHM to convol is less than zero. convolved $[i]=f l u x[i]$

else:

\#Para WAGGS :

sigma $=$ fwhm_convol/(2.355)

gauss $=$ ss.gaussian $(2 *$ filter_width +1, sigma $)$

flux_window $=$ inter_flux $\left[\left(i-f i l t e r_{-}\right.\right.$width $):\left(i+f i l t e r_{-}\right.$width $\left.)\right]$

err_window $=$ inter_err $\left[\left(i-f i l t e r \_w i d t h\right):\left(i+f i l t e r \_w i d t h\right)\right]$

\#print(flux_window)

fold = ss.convolve(flux_window, gauss, method='auto') / sum(gauss)

fold2 = ss.convolve(err_window, gauss, method='auto') / sum(gauss)

convolved $=$ fold $[$ int $(\operatorname{len}($ fold $) / 2)]$

e_convolved $=$ fold $2[\operatorname{int}(\operatorname{len}($ fold $) / 2)]$

arq.write( $\% 4.3 f \quad \%+1.5 e \quad \% 1.5 e \backslash n$ ' $\%$ (extrap_lambda[i], convolved, e_convolved))

$i+=1$

$\operatorname{print}(j)$

$j+=1$ 
Apêndice C

\section{Resultados do ajuste em $R_{W}$}

Tabela C.1 - Resultados do ajuste em $R_{W}$ e incertezas provenientes do ajuste de espectros perturbados da biblioteca WAGGS.

\begin{tabular}{|c|c|c|c|c|c|c|}
\hline Aglomerado & $\log \left(t_{\text {light }}\right)$ & $\log \left(t_{\text {mass }}\right)$ & {$[\mathrm{Fe} / \mathrm{H}]_{\text {light }}$} & {$[\mathrm{Fe} / \mathrm{H}]_{\text {mass }}$} & {$[\alpha / F e]_{\text {light }}$} & {$[\alpha / F e]_{\text {mass }}$} \\
\hline Fornax3_B7000_2015-08-09 & $9.512 \pm 0.002$ & $9.726 \pm 0.001$ & $-1.96 \pm 0.02$ & $-1.91 \pm 0.01$ & $0.36 \pm 0.01$ & $0.40 \pm 0.00$ \\
\hline Fornax4_B7000_2015-08-10 & $9.905 \pm 0.001$ & $9.916 \pm 0.001$ & $-1.35 \pm 0.04$ & $-1.30 \pm 0.03$ & $0.04 \pm 0.08$ & $0.06 \pm 0.08$ \\
\hline Fornax5_B7000_2015-08-11 & $9.685 \pm 0.004$ & $9.793 \pm 0.003$ & $-1.89 \pm 0.06$ & $-1.85 \pm 0.04$ & $0.39 \pm 0.03$ & $0.40 \pm 0.03$ \\
\hline NGC0104_B7000_2015-01-30 & $9.952 \pm 0.002$ & $10.038 \pm 0.000$ & $-0.50 \pm 0.01$ & $-0.32 \pm 0.01$ & $0.38 \pm 0.01$ & $0.38 \pm 0.01$ \\
\hline NGC0121_B7000_2015-08-11 & $10.022 \pm 0.000$ & $10.023 \pm 0.000$ & $-1.38 \pm 0.01$ & $-1.36 \pm 0.01$ & $0.19 \pm 0.03$ & $0.18 \pm 0.03$ \\
\hline NGC0330_B7000_2015-11-02 & $8.409 \pm 0.004$ & & & & $0.29 \pm 0.08$ & $0.19 \pm 0.08$ \\
\hline NGC0361_B7000_2015-11-02 & $9.975 \pm 0.003$ & $9.987 \pm 0.002$ & & & $0.19 \pm 0.07$ & $0.21 \pm 0.06$ \\
\hline NGC0362_B7000_2015-01-30 & $9.640 \pm 0.004$ & $9.949 \pm 0.000$ & $-0.92 \pm 0.04$ & $-0.94 \pm 0.02$ & $0.35 \pm 0.02$ & $0.40 \pm 0.04$ \\
\hline NGC0416_B7000_2015-08-08 & $9.665 \pm 0.004$ & $9.870 \pm 0.001$ & $-1.13 \pm 0.06$ & $-1.18 \pm 0.04$ & $0.18 \pm 0.03$ & $0.23 \pm 0.03$ \\
\hline NGC0419_B7000_2015-12-18 & & & & & & $0.11 \pm 0.02$ \\
\hline NGC1261_B7000_2015-08-08 & $9.915 \pm 0.001$ & $9.925 \pm 0.000$ & $-1.22 \pm 0.01$ & $-1.16 \pm 0.01$ & $0.34 \pm 0.02$ & $0.35 \pm 0.02$ \\
\hline NGC1783_B7000_2016-04-02 & $9.055 \pm 0.003$ & & $-0.51 \pm 0.07$ & $-0.47 \pm 0.04$ & $0.16 \pm 0.04$ & $0.15 \pm 0.05$ \\
\hline NGC1786_B7000_2016-10-03 & $9.598 \pm 0.004$ & & $-1.80 \pm 0.03$ & $-1.76 \pm 0.03$ & $0.16 \pm 0.06$ & $0.16 \pm 0.06$ \\
\hline NGC1846_B7000_2016-04-01 & & & & & & $0.35 \pm 0.16$ \\
\hline NGC1846_B7000_2016-04-02 & $8.813 \pm 0.010$ & $9.741 \pm 0.010$ & $-0.43 \pm 0.15$ & $-0.41 \pm 0.16$ & $0.06 \pm 0.04$ & $0.04 \pm 0.09$ \\
\hline NGC1850_B7000_2016-04-01 & $8.282 \pm 0.003$ & & & & $0.20 \pm 0.07$ & $0.18 \pm 0.07$ \\
\hline NGC1851_B7000_2015-01-31 & $9.746 \pm 0.003$ & & $-0.93 \pm 0.03$ & $-0.93 \pm 0.01$ & $0.36 \pm 0.01$ & $0.40 \pm 0.02$ \\
\hline NGC1856_B7000_2016-04-02 & $8.448 \pm 0.002$ & $8.473 \pm 0.002$ & $-0.51 \pm 0.08$ & $-0.53 \pm 0.07$ & $0.17 \pm 0.05$ & $0.19 \pm 0.05$ \\
\hline NGC1866_B7000_2016-04-02 & $8.242 \pm 0.002$ & $8.283 \pm 0.003$ & $-0.39 \pm 0.06$ & $-0.39 \pm 0.08$ & $0.12 \pm 0.04$ & $0.10 \pm 0.05$ \\
\hline NGC1868_B7000_2016-10-03 & $8.932 \pm 0.004$ & $9.024 \pm 0.004$ & $-0.59 \pm 0.06$ & $-0.71 \pm 0.13$ & $0.15 \pm 0.05$ & $0.15 \pm 0.05$ \\
\hline NGC1898_B7000_2016-10-03 & $9.881 \pm 0.002$ & $9.905 \pm 0.001$ & $-1.30 \pm 0.07$ & $-1.18 \pm 0.05$ & $0.06 \pm 0.07$ & $0.08 \pm 0.06$ \\
\hline NGC1904_B7000_2015-01-30 & $9.825 \pm 0.001$ & $9.881 \pm 0.000$ & $-1.56 \pm 0.01$ & $-1.51 \pm 0.01$ & $0.23 \pm 0.03$ & $0.24 \pm 0.02$ \\
\hline NGC1916_B7000_2015-12-18 & $9.194 \pm 0.003$ & $9.436 \pm 0.003$ & $-1.37 \pm 0.05$ & $-1.18 \pm 0.07$ & $0.14 \pm 0.04$ & $0.19 \pm 0.05$ \\
\hline NGC1978_B7000_2016-10-03 & $9.307 \pm 0.006$ & $9.760 \pm 0.004$ & $-0.72 \pm 0.03$ & $-0.76 \pm 0.06$ & $0.13 \pm 0.03$ & $0.17 \pm 0.05$ \\
\hline
\end{tabular}




\begin{tabular}{|c|c|c|c|c|c|c|}
\hline Aglomerado & $\log \left(t_{\text {light }}\right)$ & $\log \left(t_{\text {mass }}\right)$ & {$[\mathrm{Fe} / H]_{\text {light }}$} & {$[\mathrm{Fe} / \mathrm{H}]_{\text {mass }}$} & {$[\alpha / F e]_{\text {light }}$} & {$[\alpha / F e]_{\text {mass }}$} \\
\hline NGC2004_B7000_2016-09-30 & $8.613 \pm 0.013$ & $9.923 \pm 0.010$ & $-1.05 \pm 0.14$ & $-0.74 \pm 0.23$ & $0.28 \pm 0.06$ & $0.31 \pm 0.06$ \\
\hline NGC2019_B7000_2016-09-30 & $9.852 \pm 0.002$ & $9.861 \pm 0.001$ & $-1.41 \pm 0.03$ & $-1.41 \pm 0.04$ & $0.12 \pm 0.05$ & $0.11 \pm 0.06$ \\
\hline NGC2100_B7000_2015-12-17 & $8.490 \pm 0.004$ & $9.451 \pm 0.003$ & $-2.16 \pm 0.04$ & $-2.01 \pm 0.04$ & $0.24 \pm 0.07$ & $0.37 \pm 0.02$ \\
\hline NGC2136_B7000_2016-10-03 & $8.277 \pm 0.005$ & $8.711 \pm 0.008$ & $-0.55 \pm 0.10$ & $-0.69 \pm 0.15$ & $0.02 \pm 0.05$ & $0.02 \pm 0.07$ \\
\hline NGC2808_B7000_2015-01-31 & $9.730 \pm 0.004$ & $9.929 \pm 0.000$ & $-0.90 \pm 0.02$ & $-0.94 \pm 0.01$ & $0.34 \pm 0.01$ & $0.36 \pm 0.02$ \\
\hline NGC2808_B7000_2015-07-10 & $9.668 \pm 0.003$ & $9.922 \pm 0.000$ & $-0.99 \pm 0.05$ & $-0.95 \pm 0.01$ & $0.25 \pm 0.01$ & $0.25 \pm 0.01$ \\
\hline NGC3201_B7000_2015-04-15 & $9.728 \pm 0.003$ & $9.937 \pm 0.001$ & $-1.23 \pm 0.02$ & $-1.23 \pm 0.03$ & $0.27 \pm 0.02$ & $0.31 \pm 0.02$ \\
\hline NGC4147_B7000_2015-01-30 & $9.468 \pm 0.005$ & $9.704 \pm 0.004$ & $-1.66 \pm 0.07$ & $-1.70 \pm 0.06$ & $0.35 \pm 0.06$ & $0.36 \pm 0.06$ \\
\hline NGC4590_B7000_2015-04-13 & $9.834 \pm 0.001$ & $9.844 \pm 0.000$ & $-1.91 \pm 0.01$ & $-1.88 \pm 0.01$ & $0.40 \pm 0.00$ & $0.40 \pm 0.00$ \\
\hline NGC4833_B7000_2015-01-30 & $9.353 \pm 0.001$ & $9.673 \pm 0.002$ & $-1.78 \pm 0.03$ & $-1.81 \pm 0.01$ & $0.40 \pm 0.01$ & $0.40 \pm 0.00$ \\
\hline NGC5024_B7000_2015-01-30 & $9.907 \pm 0.002$ & $9.924 \pm 0.002$ & $-1.80 \pm 0.02$ & $-1.77 \pm 0.05$ & $0.32 \pm 0.03$ & $0.30 \pm 0.03$ \\
\hline NGC5139_B7000_2015-04-13 & $9.952 \pm 0.001$ & $9.962 \pm 0.001$ & $-1.50 \pm 0.02$ & $-1.47 \pm 0.05$ & $0.40 \pm 0.00$ & $0.40 \pm 0.00$ \\
\hline NGC5272_B7000_2016-04-02 & $9.892 \pm 0.001$ & $9.908 \pm 0.001$ & $-1.43 \pm 0.01$ & $-1.36 \pm 0.02$ & $0.24 \pm 0.01$ & $0.27 \pm 0.01$ \\
\hline NGC5286_B7000_2015-04-14 & $9.929 \pm 0.001$ & $9.939 \pm 0.001$ & $-1.51 \pm 0.01$ & $-1.46 \pm 0.02$ & $0.33 \pm 0.02$ & $0.33 \pm 0.02$ \\
\hline NGC5634_B7000_2015-08-10 & $9.710 \pm 0.001$ & $9.835 \pm 0.000$ & $-1.59 \pm 0.01$ & $-1.59 \pm 0.01$ & $0.33 \pm 0.01$ & $0.33 \pm 0.01$ \\
\hline NGC5694_B7000_2015-08-11 & $9.731 \pm 0.001$ & $9.801 \pm 0.000$ & $-1.80 \pm 0.01$ & $-1.79 \pm 0.00$ & $0.26 \pm 0.01$ & $0.26 \pm 0.01$ \\
\hline NGC5824_B7000_2016-04-01 & $9.856 \pm 0.001$ & $9.857 \pm 0.001$ & $-1.79 \pm 0.02$ & $-1.79 \pm 0.02$ & $0.32 \pm 0.03$ & $0.33 \pm 0.03$ \\
\hline NGC5904_B7000_2015-04-15 & $9.706 \pm 0.002$ & $9.929 \pm 0.000$ & $-1.15 \pm 0.02$ & $-1.10 \pm 0.02$ & $0.36 \pm 0.01$ & $0.40 \pm 0.01$ \\
\hline NGC5927_B7000_2015-01-31 & $10.098 \pm 0.000$ & $10.106 \pm 0.000$ & $-0.14 \pm 0.01$ & $-0.06 \pm 0.01$ & $0.28 \pm 0.01$ & $0.31 \pm 0.01$ \\
\hline NGC5986_B7000_2015-07-10 & $9.817 \pm 0.002$ & $9.884 \pm 0.000$ & $-1.38 \pm 0.01$ & $-1.37 \pm 0.01$ & $0.29 \pm 0.02$ & $0.29 \pm 0.01$ \\
\hline NGC6093_B7000_2015-04-14 & $9.860 \pm 0.006$ & $9.962 \pm 0.003$ & $-1.61 \pm 0.05$ & $-1.59 \pm 0.07$ & $0.32 \pm 0.07$ & $0.34 \pm 0.06$ \\
\hline NGC6121_B7000_2015-04-15 & $9.906 \pm 0.003$ & $9.944 \pm 0.002$ & $-1.14 \pm 0.02$ & $-1.13 \pm 0.05$ & $0.39 \pm 0.00$ & $0.40 \pm 0.00$ \\
\hline NGC6139_B7000_2015-08-11 & $10.016 \pm 0.002$ & $10.028 \pm 0.001$ & $-1.35 \pm 0.02$ & $-1.30 \pm 0.05$ & $0.40 \pm 0.01$ & $0.40 \pm 0.00$ \\
\hline NGC6171_B7000_2015-07-09 & $10.030 \pm 0.000$ & $10.042 \pm 0.000$ & $-0.70 \pm 0.01$ & $-0.63 \pm 0.01$ & $0.40 \pm 0.00$ & $0.40 \pm 0.00$ \\
\hline NGC6218_B7000_2015-07-09 & $9.607 \pm 0.004$ & $9.820 \pm 0.002$ & $-1.00 \pm 0.02$ & $-0.97 \pm 0.03$ & $0.37 \pm 0.02$ & $0.40 \pm 0.00$ \\
\hline NGC6254_B7000_2015-04-13 & $9.940 \pm 0.001$ & $9.955 \pm 0.001$ & $-1.38 \pm 0.02$ & $-1.34 \pm 0.03$ & $0.38 \pm 0.03$ & $0.38 \pm 0.02$ \\
\hline NGC6266_B7000_2015-08-09 & $9.955 \pm 0.002$ & $9.955 \pm 0.000$ & $-0.88 \pm 0.01$ & $-0.87 \pm 0.01$ & $0.38 \pm 0.01$ & $0.38 \pm 0.01$ \\
\hline NGC6273_B7000_2015-08-10 & $9.913 \pm 0.001$ & $9.919 \pm 0.001$ & $-1.43 \pm 0.02$ & $-1.36 \pm 0.03$ & $0.38 \pm 0.02$ & $0.38 \pm 0.02$ \\
\hline NGC6284_B7000_2016-04-01 & $9.972 \pm 0.002$ & $9.979 \pm 0.001$ & $-1.02 \pm 0.03$ & $-0.95 \pm 0.03$ & $0.40 \pm 0.02$ & $0.40 \pm 0.02$ \\
\hline NGC6284_B7000_2016-09-29 & $9.838 \pm 0.002$ & $9.857 \pm 0.002$ & $-1.07 \pm 0.04$ & $-0.89 \pm 0.06$ & $0.40 \pm 0.01$ & $0.40 \pm 0.02$ \\
\hline NGC6293_B7000_2015-08-11 & $9.674 \pm 0.002$ & $9.769 \pm 0.000$ & $-1.84 \pm 0.01$ & $-1.82 \pm 0.01$ & $0.40 \pm 0.01$ & $0.40 \pm 0.00$ \\
\hline NGC6304_B7000_2015-04-14 & $10.064 \pm 0.001$ & $10.082 \pm 0.001$ & $-0.31 \pm 0.03$ & $-0.09 \pm 0.04$ & $0.34 \pm 0.03$ & $0.34 \pm 0.02$ \\
\hline NGC6304_B7000_2015-08-08 & $10.105 \pm 0.001$ & $10.106 \pm 0.001$ & $-0.19 \pm 0.01$ & $-0.12 \pm 0.03$ & $0.31 \pm 0.01$ & $0.32 \pm 0.01$ \\
\hline NGC6316_B7000_2016-04-02 & $10.057 \pm 0.000$ & $10.067 \pm 0.000$ & $-0.43 \pm 0.01$ & $-0.30 \pm 0.02$ & $0.39 \pm 0.01$ & $0.39 \pm 0.01$ \\
\hline NGC6333_B7000_2016-04-02 & $9.943 \pm 0.001$ & $9.956 \pm 0.001$ & $-1.62 \pm 0.01$ & $-1.59 \pm 0.02$ & $0.27 \pm 0.02$ & $0.27 \pm 0.02$ \\
\hline NGC6342_B7000_2015-07-10 & $10.031 \pm 0.001$ & $10.041 \pm 0.001$ & $-0.49 \pm 0.02$ & $-0.35 \pm 0.04$ & $0.37 \pm 0.01$ & $0.35 \pm 0.02$ \\
\hline NGC6342_B7000_2015-08-10 & $9.953 \pm 0.001$ & $9.954 \pm 0.001$ & $-0.48 \pm 0.03$ & $-0.33 \pm 0.05$ & $0.39 \pm 0.02$ & $0.37 \pm 0.02$ \\
\hline
\end{tabular}




\begin{tabular}{|c|c|c|c|c|c|c|}
\hline Aglomerado & $\log \left(t_{\text {light }}\right)$ & $\log \left(t_{\text {mass }}\right)$ & {$[\mathrm{Fe} / \mathrm{H}]_{\text {light }}$} & {$[\mathrm{Fe} / \mathrm{H}]_{\text {mass }}$} & {$[\alpha / F e]_{\text {light }}$} & {$[\alpha / F e]_{\text {mass }}$} \\
\hline NGC6352_B7000_2015-07-09 & $10.072 \pm 0.000$ & $10.091 \pm 0.000$ & $-0.33 \pm 0.04$ & $-0.15 \pm 0.03$ & $0.34 \pm 0.03$ & $0.32 \pm 0.02$ \\
\hline NGC6356_B7000_2016-04-01 & $10.066 \pm 0.000$ & $10.085 \pm 0.000$ & $-0.38 \pm 0.01$ & $-0.21 \pm 0.02$ & $0.38 \pm 0.01$ & $0.35 \pm 0.01$ \\
\hline NGC6362_B7000_2015-08-11 & $10.104 \pm 0.003$ & $10.127 \pm 0.000$ & $-0.48 \pm 0.03$ & $-0.39 \pm 0.02$ & $0.40 \pm 0.02$ & $0.40 \pm 0.01$ \\
\hline NGC6388_B7000_2015-04-13 & $9.944 \pm 0.002$ & $9.969 \pm 0.000$ & $-0.47 \pm 0.01$ & $-0.44 \pm 0.01$ & $0.18 \pm 0.01$ & $0.18 \pm 0.01$ \\
\hline NGC6397_B7000_2015-04-13 & $9.203 \pm 0.001$ & $9.435 \pm 0.001$ & $-1.96 \pm 0.03$ & $-1.97 \pm 0.02$ & $0.40 \pm 0.01$ & $0.40 \pm 0.00$ \\
\hline NGC6440_B7000_2016-10-03 & $10.122 \pm 0.001$ & $10.124 \pm 0.001$ & $-0.23 \pm 0.04$ & $-0.13 \pm 0.04$ & $0.34 \pm 0.03$ & $0.31 \pm 0.03$ \\
\hline NGC6441_B7000_2015-04-15 & $10.033 \pm 0.002$ & $10.041 \pm 0.000$ & $-0.38 \pm 0.01$ & $-0.23 \pm 0.01$ & $0.29 \pm 0.01$ & $0.28 \pm 0.01$ \\
\hline NGC6522_B7000_2016-04-02 & $9.866 \pm 0.001$ & $9.974 \pm 0.001$ & $-1.06 \pm 0.01$ & $-0.95 \pm 0.03$ & $0.40 \pm 0.01$ & $0.40 \pm 0.00$ \\
\hline NGC6528_B7000_2015-04-13 & $10.099 \pm 0.002$ & $10.103 \pm 0.002$ & $0.05 \pm 0.03$ & $0.10 \pm 0.03$ & $0.13 \pm 0.02$ & $0.15 \pm 0.02$ \\
\hline NGC6541_B7000_2015-04-14 & $9.866 \pm 0.002$ & $9.906 \pm 0.000$ & $-1.65 \pm 0.01$ & $-1.60 \pm 0.01$ & $0.33 \pm 0.01$ & $0.33 \pm 0.01$ \\
\hline NGC6553_B7000_2015-04-15 & $10.126 \pm 0.001$ & $10.127 \pm 0.001$ & $0.01 \pm 0.02$ & $0.06 \pm 0.02$ & $0.22 \pm 0.03$ & $0.23 \pm 0.02$ \\
\hline NGC6569_B7000_2015-08-10 & $10.042 \pm 0.000$ & $10.047 \pm 0.000$ & $-0.66 \pm 0.02$ & $-0.57 \pm 0.03$ & $0.38 \pm 0.01$ & $0.39 \pm 0.01$ \\
\hline NGC6584_B7000_2015-08-08 & $9.905 \pm 0.002$ & $9.921 \pm 0.001$ & $-1.21 \pm 0.03$ & $-1.13 \pm 0.04$ & $0.34 \pm 0.05$ & $0.36 \pm 0.05$ \\
\hline NGC6624_B7000_2015-07-09 & $10.099 \pm 0.000$ & $10.103 \pm 0.000$ & $-0.39 \pm 0.01$ & $-0.37 \pm 0.01$ & $0.37 \pm 0.01$ & $0.37 \pm 0.00$ \\
\hline NGC6637_B7000_2015-07-09 & $9.966 \pm 0.001$ & $10.040 \pm 0.000$ & $-0.41 \pm 0.01$ & $-0.38 \pm 0.01$ & $0.40 \pm 0.00$ & $0.40 \pm 0.00$ \\
\hline NGC6652_B7000_2015-08-08 & $9.930 \pm 0.001$ & $9.922 \pm 0.001$ & $-0.65 \pm 0.01$ & $-0.60 \pm 0.03$ & $0.40 \pm 0.01$ & $0.40 \pm 0.01$ \\
\hline NGC6656_B7000_2015-07-09 & $9.830 \pm 0.002$ & $9.858 \pm 0.001$ & $-1.54 \pm 0.02$ & $-1.54 \pm 0.02$ & & $0.26 \pm 0.03$ \\
\hline NGC6681_B7000_2015-08-10 & $9.805 \pm 0.002$ & $9.870 \pm 0.000$ & $-1.46 \pm 0.02$ & $-1.42 \pm 0.03$ & $0.37 \pm 0.02$ & $0.37 \pm 0.02$ \\
\hline NGC6715_B7000_2015-07-09 & $9.748 \pm 0.002$ & $9.908 \pm 0.000$ & $-1.13 \pm 0.03$ & $-1.05 \pm 0.03$ & $0.17 \pm 0.02$ & $0.16 \pm 0.01$ \\
\hline NGC6717_B7000_2015-04-15 & $9.321 \pm 0.009$ & $10.013 \pm 0.001$ & $-0.79 \pm 0.06$ & $-0.56 \pm 0.06$ & $0.29 \pm 0.04$ & $0.35 \pm 0.04$ \\
\hline NGC6717_B7000_2016-04-01 & $9.613 \pm 0.007$ & $10.114 \pm 0.000$ & $-0.87 \pm 0.05$ & $-0.68 \pm 0.04$ & $0.27 \pm 0.04$ & $0.33 \pm 0.05$ \\
\hline NGC6723_B7000_2016-09-29 & $9.987 \pm 0.001$ & $10.004 \pm 0.001$ & $-0.82 \pm 0.01$ & $-0.68 \pm 0.03$ & $0.40 \pm 0.00$ & $0.40 \pm 0.00$ \\
\hline NGC6752_B7000_2015-04-14 & $9.889 \pm 0.001$ & $9.899 \pm 0.000$ & $-1.49 \pm 0.00$ & $-1.42 \pm 0.01$ & $0.34 \pm 0.01$ & $0.35 \pm 0.01$ \\
\hline NGC6809_B7000_2015-08-09 & $9.665 \pm 0.001$ & $9.822 \pm 0.001$ & $-1.82 \pm 0.01$ & $-1.79 \pm 0.00$ & $0.37 \pm 0.01$ & $0.40 \pm 0.00$ \\
\hline NGC6838_B7000_2015-08-08 & $9.917 \pm 0.001$ & $9.979 \pm 0.001$ & $-0.62 \pm 0.02$ & $-0.49 \pm 0.02$ & $0.40 \pm 0.00$ & $0.40 \pm 0.01$ \\
\hline NGC6864_B7000_2015-08-10 & $9.879 \pm 0.001$ & $9.941 \pm 0.000$ & $-0.93 \pm 0.01$ & $-0.94 \pm 0.01$ & $0.26 \pm 0.01$ & $0.27 \pm 0.01$ \\
\hline NGC6934_B7000_2015-08-08 & $9.809 \pm 0.005$ & $9.880 \pm 0.003$ & $-1.39 \pm 0.03$ & $-1.38 \pm 0.05$ & $0.32 \pm 0.05$ & $0.30 \pm 0.06$ \\
\hline NGC7006_B7000_2015-08-09 & $9.894 \pm 0.001$ & $9.909 \pm 0.002$ & $-1.45 \pm 0.03$ & $-1.37 \pm 0.04$ & $0.39 \pm 0.03$ & $0.39 \pm 0.02$ \\
\hline NGC7078_B7000_2015-07-09 & $9.383 \pm 0.002$ & $9.705 \pm 0.000$ & $-1.95 \pm 0.01$ & $-1.89 \pm 0.01$ & $0.38 \pm 0.01$ & $0.40 \pm 0.00$ \\
\hline NGC7089_B7000_2015-07-09 & $9.756 \pm 0.001$ & $9.838 \pm 0.000$ & $-1.57 \pm 0.01$ & $-1.51 \pm 0.01$ & $0.21 \pm 0.01$ & $0.24 \pm 0.01$ \\
\hline NGC7099_B7000_2015-07-09 & $9.215 \pm 0.005$ & $9.541 \pm 0.004$ & $-1.90 \pm 0.06$ & $-1.90 \pm 0.06$ & $0.36 \pm 0.03$ & $0.39 \pm 0.01$ \\
\hline NGC7099_B7000_2016-09-30 & $9.175 \pm 0.002$ & $9.427 \pm 0.002$ & $-1.94 \pm 0.04$ & $-1.94 \pm 0.03$ & $0.37 \pm 0.04$ & $0.39 \pm 0.02$ \\
\hline NGC7099_B7000_2016-10-01 & $9.225 \pm 0.001$ & $9.478 \pm 0.001$ & $-1.97 \pm 0.01$ & $-1.95 \pm 0.02$ & $0.40 \pm 0.01$ & $0.40 \pm 0.00$ \\
\hline
\end{tabular}


Apêndice D

Matrizes de correlação 


\section{Bojo clássico, AGN}

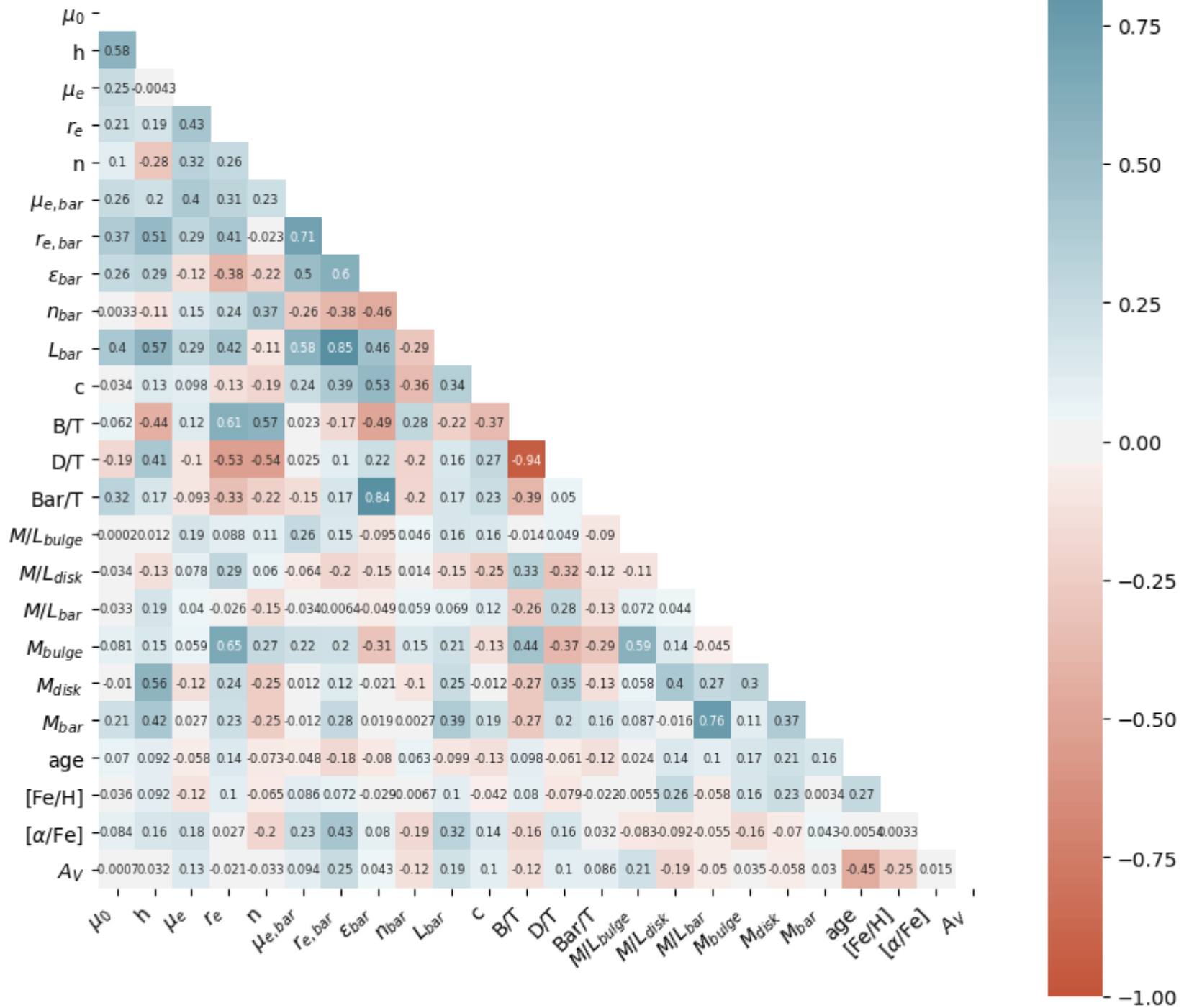

Figura D.1: Análogo à Figura 5.6 porém para galáxias de bojo clássico com AGN. 


\section{Bojo clássico, sem AGN}

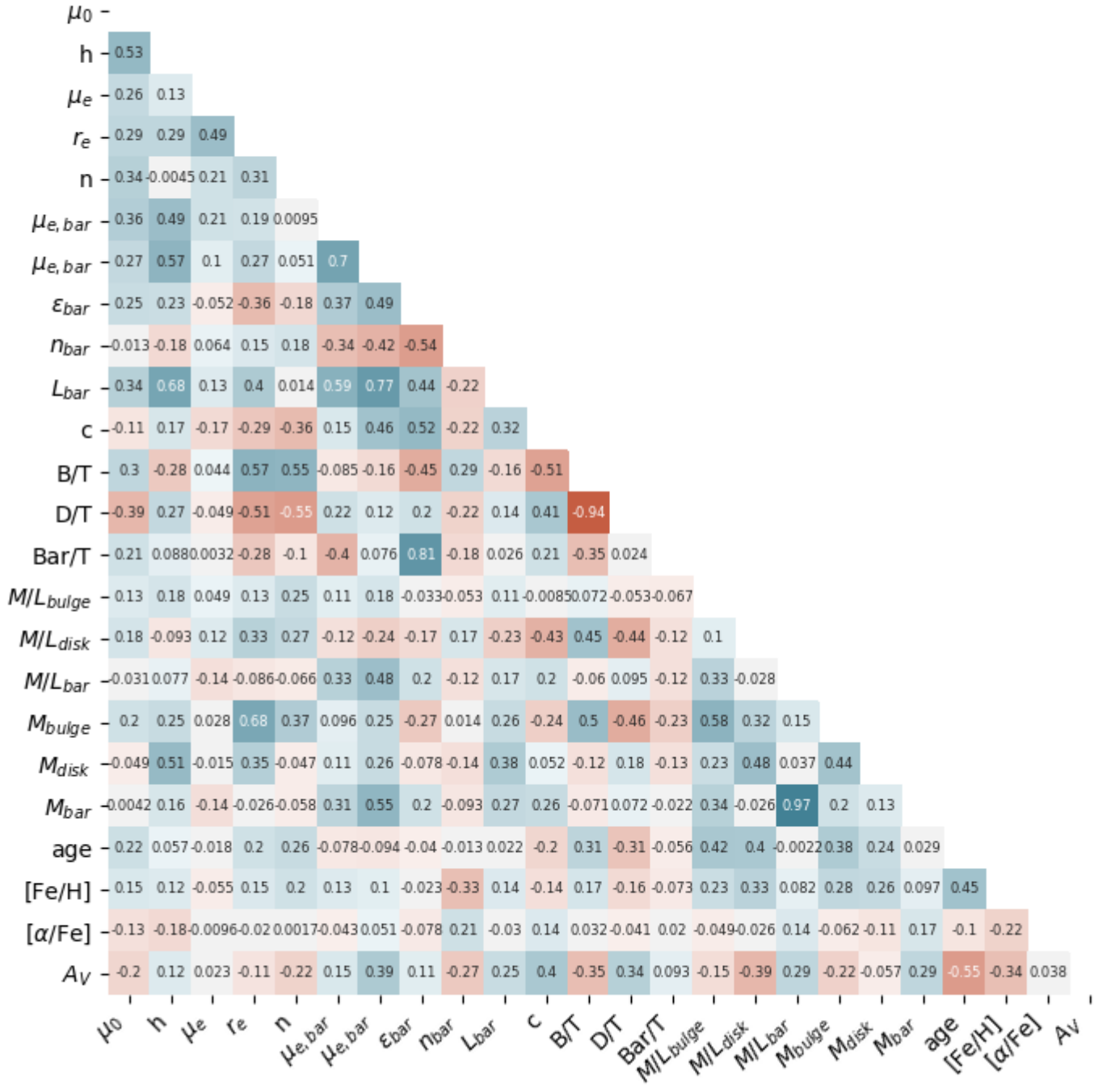

Figura D.2: Análogo à Figura 5.6 porém para galáxias de bojo clássico sem AGN. 


\section{Bojo clássico com barra, AGN}

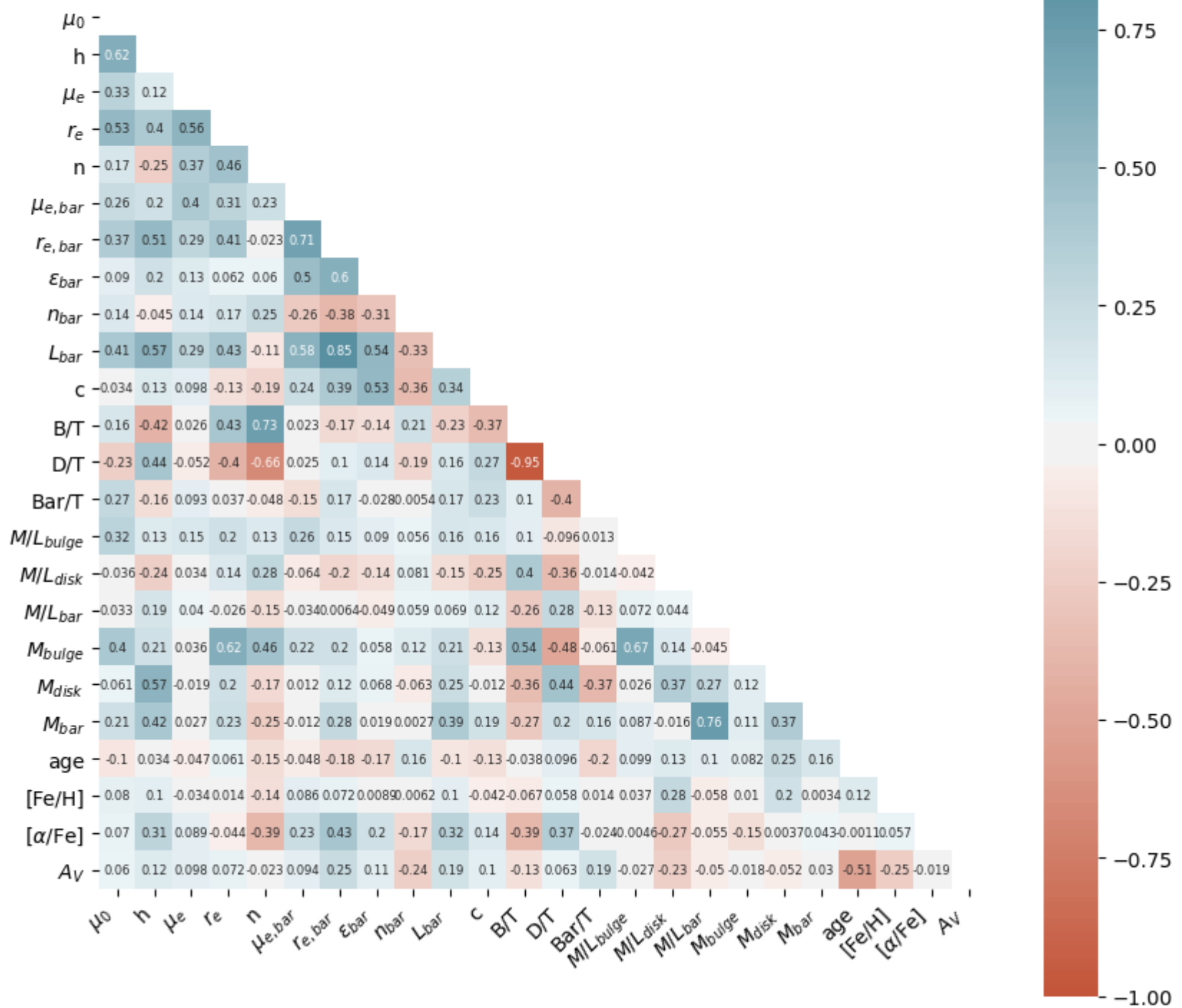

Figura D.3: Análogo à Figura 5.6 porém para galáxias de bojo clássico com barra e com AGN. 
Bojo clássico com barra, sem AGN

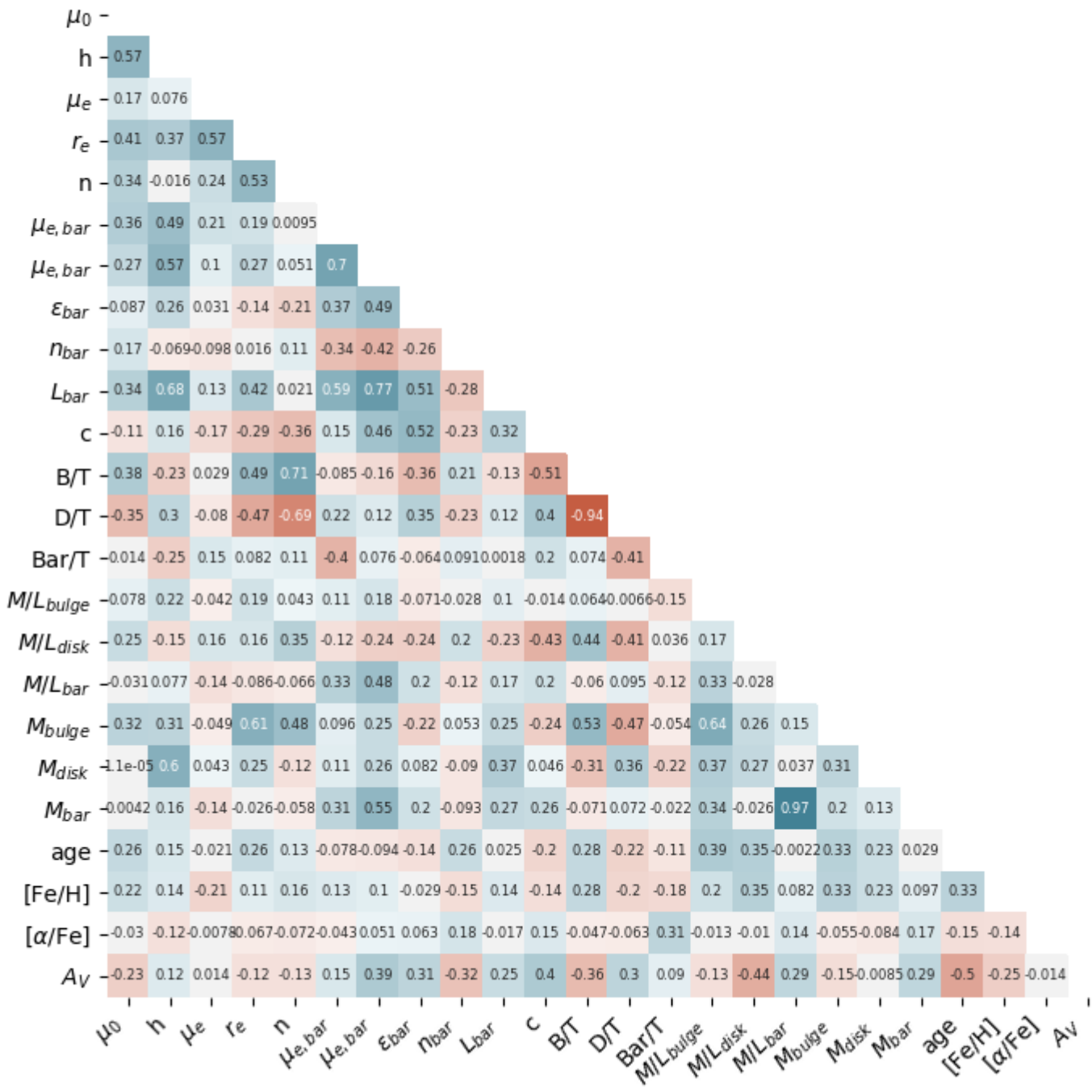

Figura D.4: Análogo à Figura 5.6 porém para galáxias de bojo clássico com barra e sem AGN. 


\section{Bojo clássico sem barra, AGN}

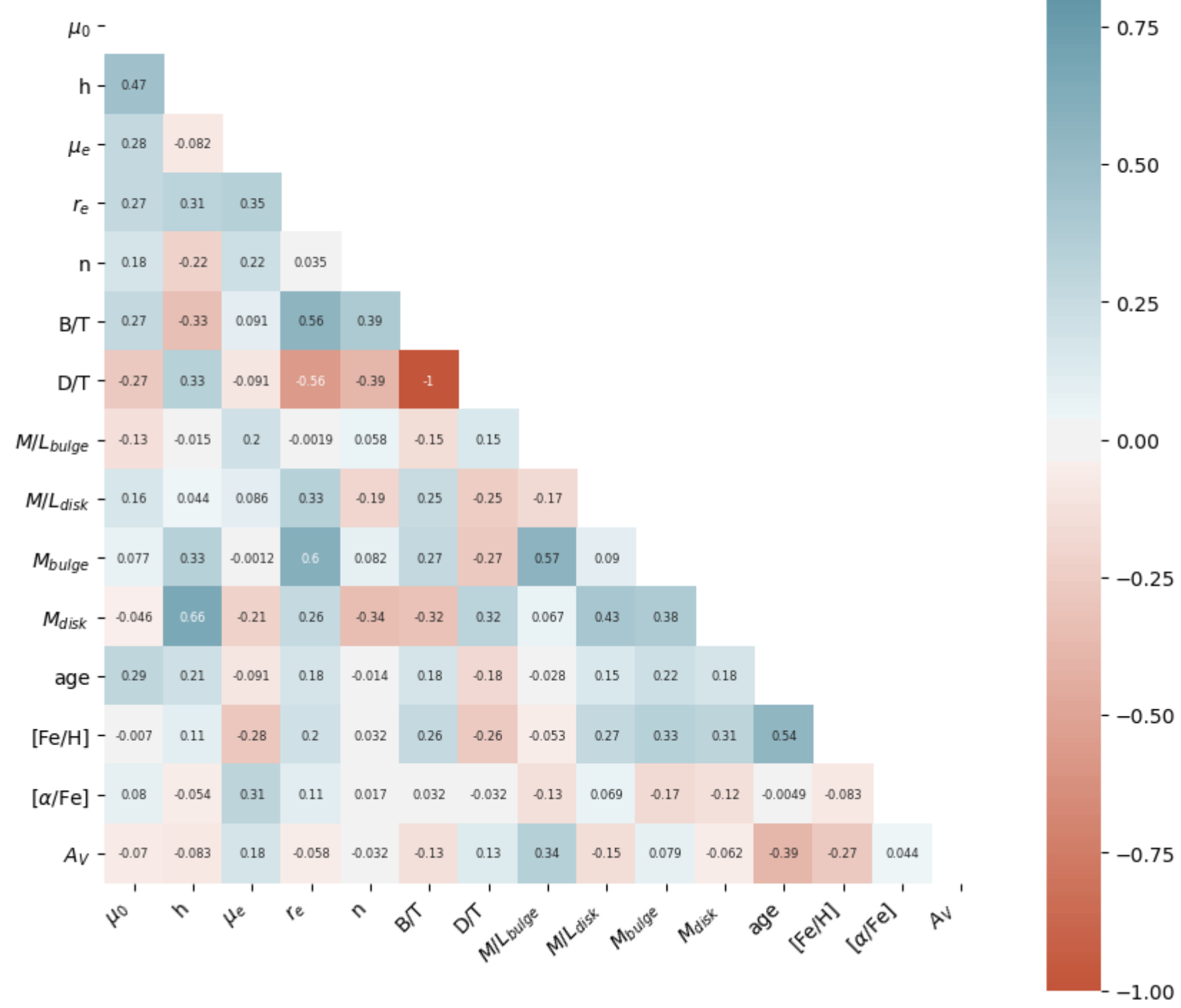

Figura D.5: Análogo à Figura 5.6 porém para galáxias de bojo clássico sem barra e com AGN. 
Bojo clássico sem barra, sem AGN

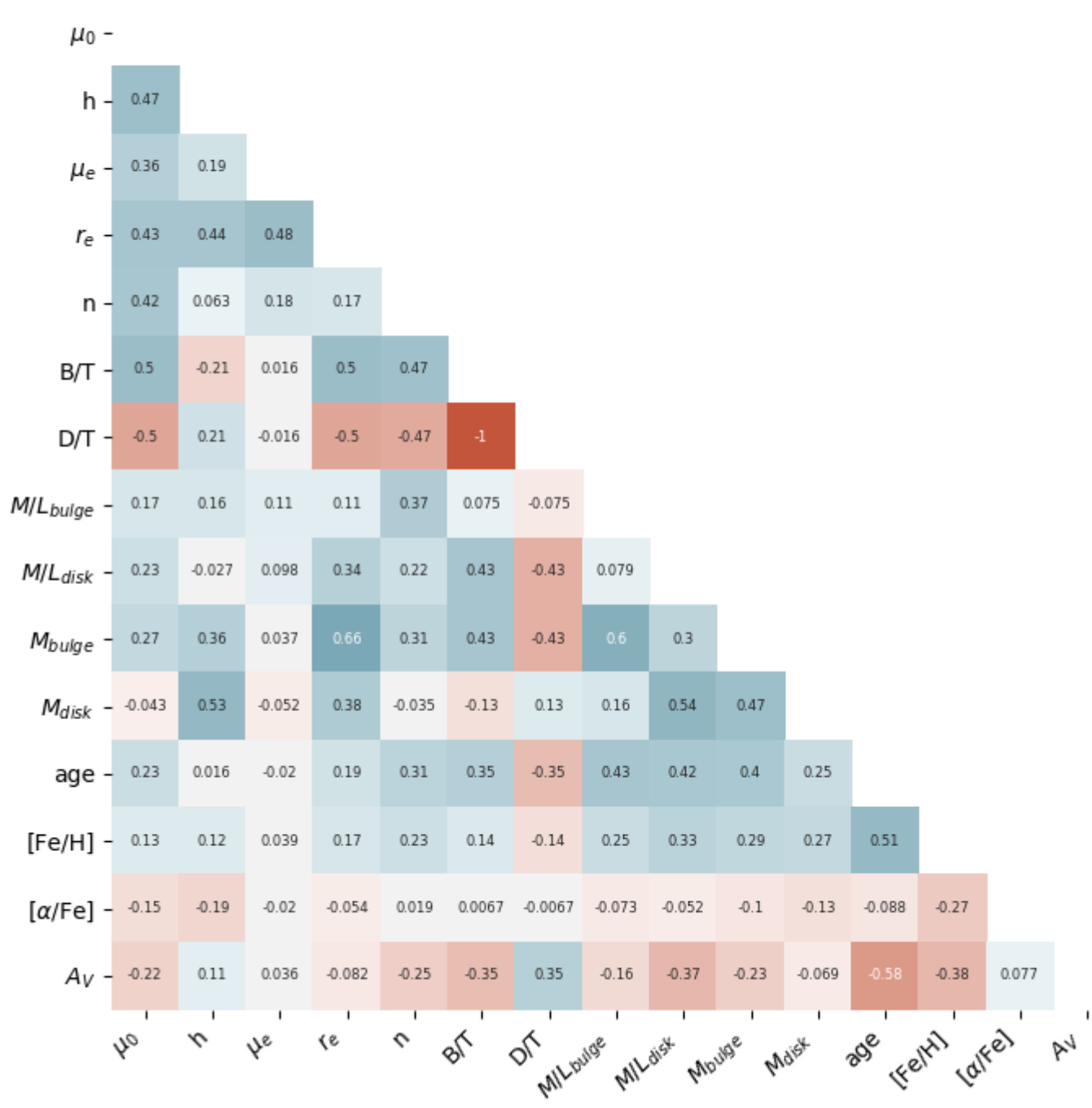

Figura D.6: Análogo à Figura 5.6 porém para galáxias de bojo clássico sem barra e sem AGN. 


\section{Pseudo bojo, AGN}

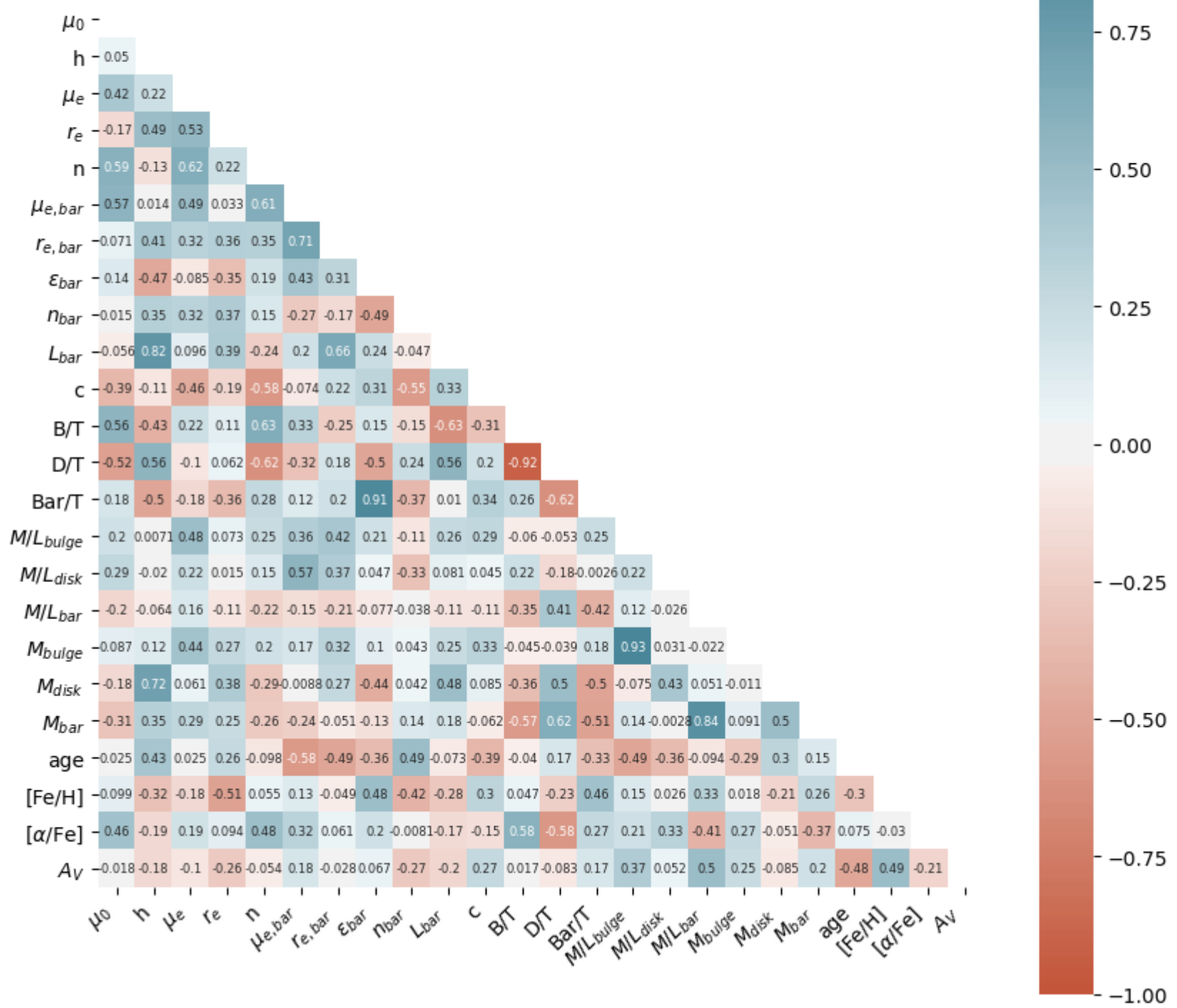

Figura D.7: Análogo à Figura 5.6 porém para galáxias de pseudo-bojo com AGN. 


\section{Pseudo bojo, sem AGN}

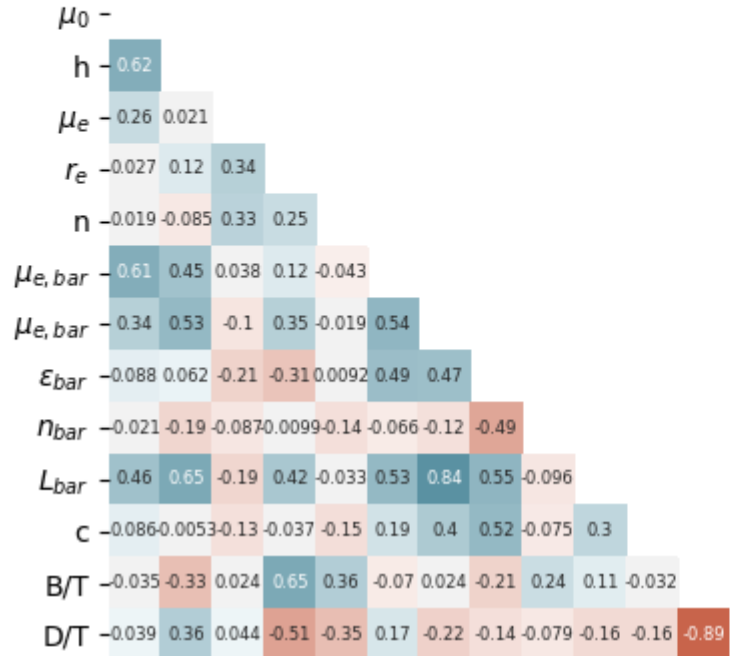

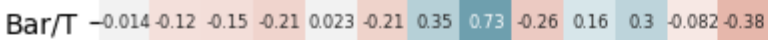

$M / L_{\text {bulge }}-0.21-0.0590 .130 .012-0.01-0.28-0.0880 .045-0.0037-0.13-0.053-0.0870 .0690 .025$

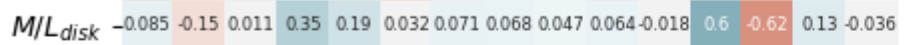

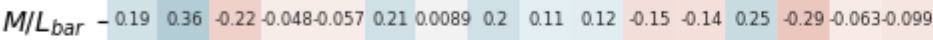

$M_{\text {bulge }}-0.2-0.0280 .096 \quad 0.22-0.022-0.2-0.038-0.0590 .033-0.059-0.083 \quad 0.020 .00490 .051 \quad 0.640 .0051-0.069$

$\begin{array}{lllllllllllllll}M_{\text {disk }}-0.014 & 0.57 & -0.12 & 0.32-0.00280 .15 & 0.37 & 0.097 & -0.21 & 0.41 & -0.092-0.0710 .079 & -0.0280 .052 & 0.34 & 0.19 & 0.12\end{array}$

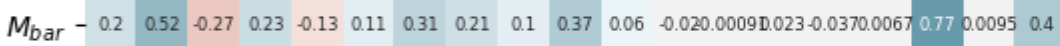

$\begin{array}{lllllllllllllllll}\text { age }-0.11 & 0.15 & 0.05 & 0.11 & 0.067 & 0.02 & -0.039 & 0.12 & -0.17 & 0.12 & -0.054 & 0.047-0.0450 .00160 .089 & 0.12 & 0.2 & 0.032 & 0.17 & 0.15\end{array}$

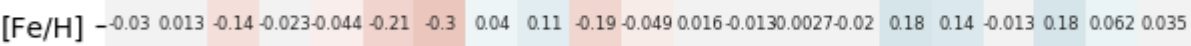

$[\alpha / \mathrm{Fe}]-0.074-0.0170 .046-0.058-0.099-0.058-0.290 .0550 .046-0.2 \quad-0.28-0.060 .0470 .0190 .017-0.078-0.0370 .023-0.074-0.077-0.11-0.11$

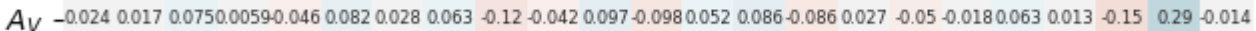

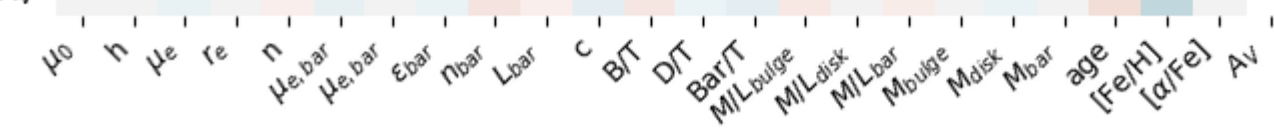

Figura D.8: Análogo à Figura 5.6. porém para galáxias de pseudo-bojo sem AGN. 


\section{Pseudo bojo com barra, AGN}

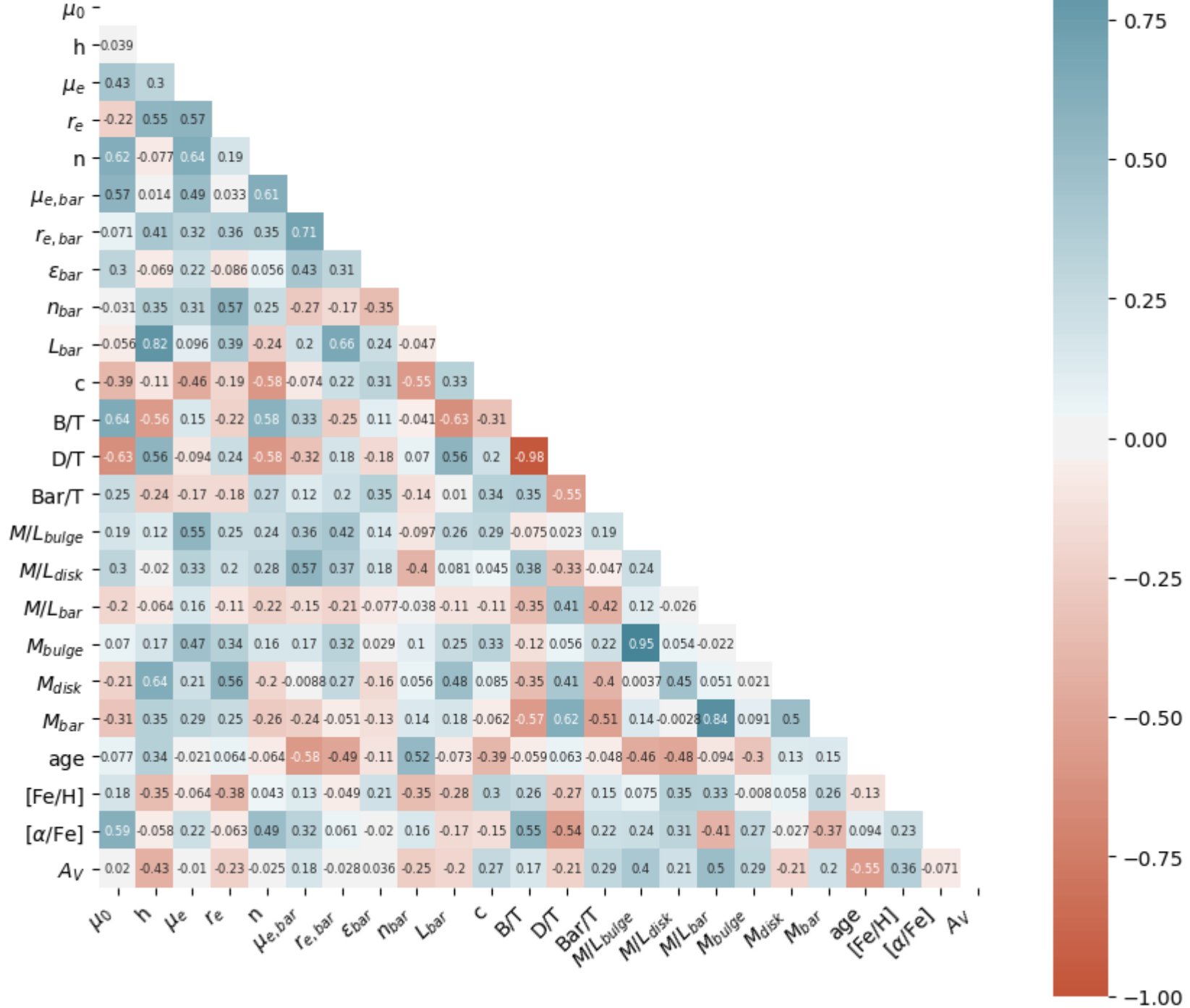

Figura D.9: Análogo à Figura 5.6 porém para galáxias de pseudo-bojo com barra e com AGN. 


\section{Pseudo bojo com barra, sem AGN}

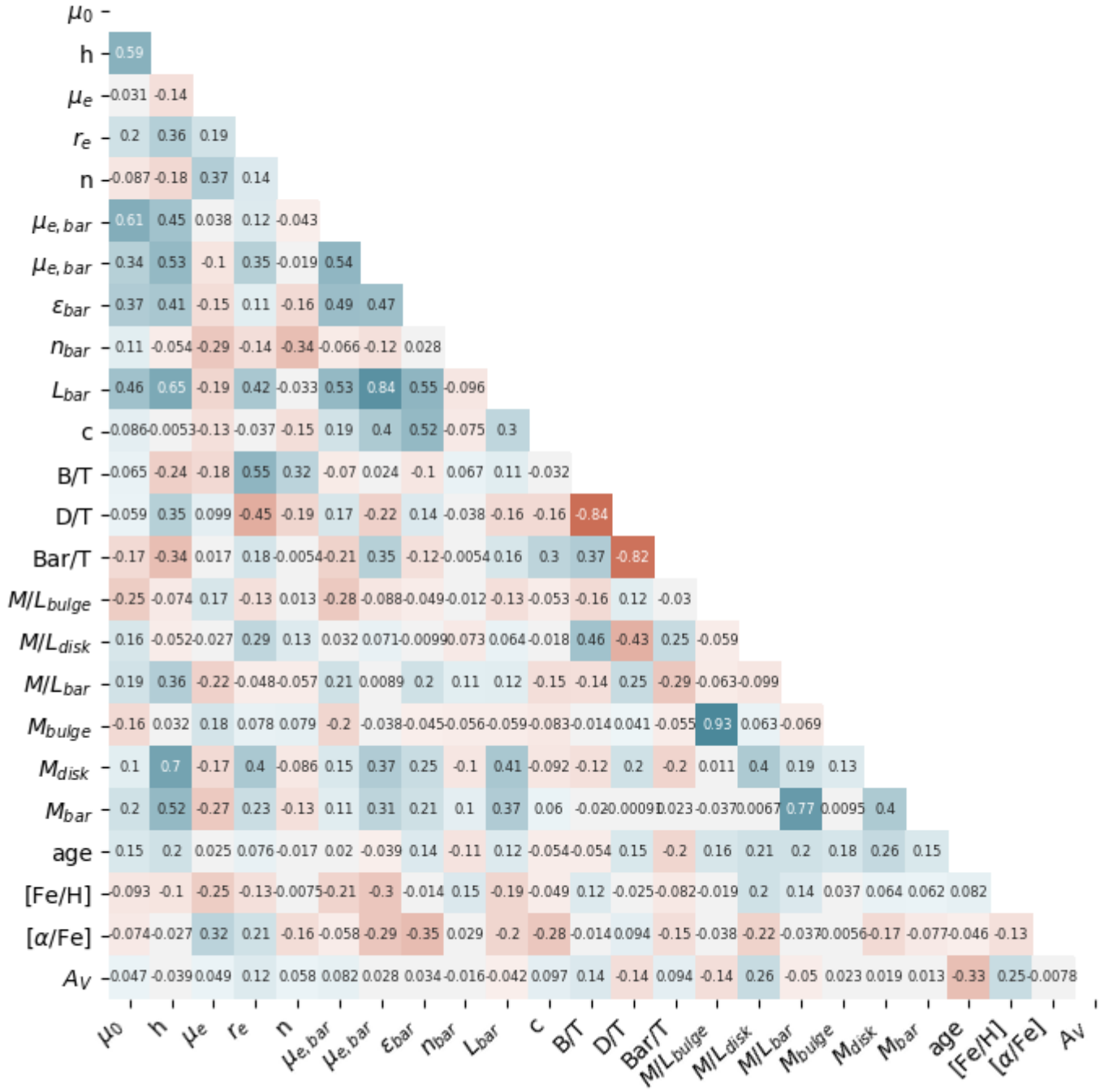

Figura D.10: Análogo à Figura 5.6 porém para galáxias de pseudo-bojo com barra e sem AGN. 
Pseudo bojo sem barra, sem AGN

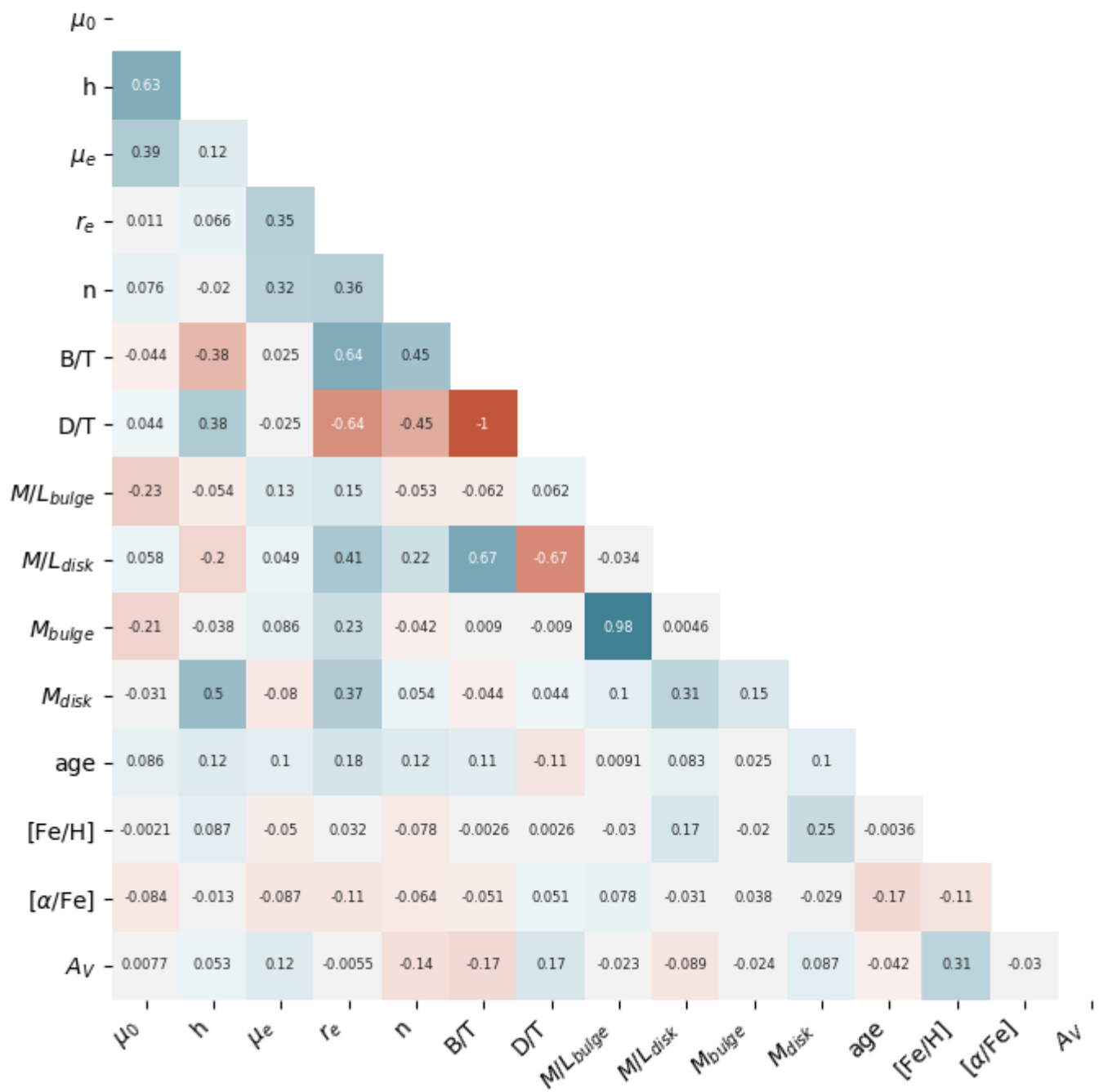

Figura D.11: Análogo à Figura 5.6 porém para galáxias de pseudo-bojo sem barra e sem AGN. 


\section{Elípticas AGN}

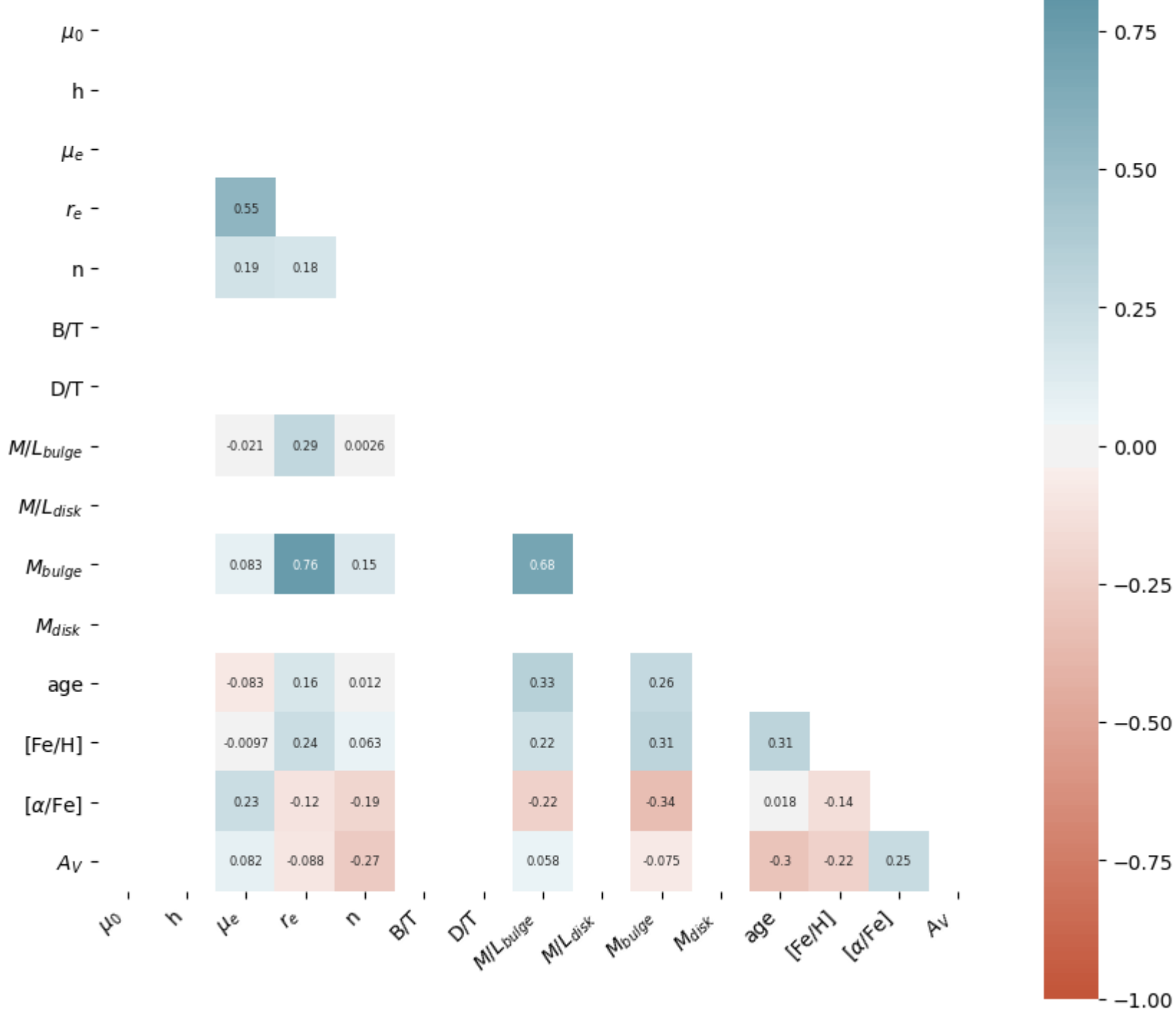

Figura D.12: Análogo à Figura 5.6 porém para galáxias elípticas com AGN. 


\section{Elípticas sem AGN}

$\mu_{0}-$

h -

$\mu_{e}-$

$r_{e}-\quad 0.53$

n-

$-0.097 \quad-0.02$

$\mathrm{B} / \mathrm{T}$ -

D/T -

$M / L_{\text {bulge - }}$

\begin{tabular}{l|l|l}
0.088 & 0.39 & 0.0054
\end{tabular}

$M / L_{\text {disk }}$ -

Mbulge -

0.065

$0.78 \quad 0.077$

0.65
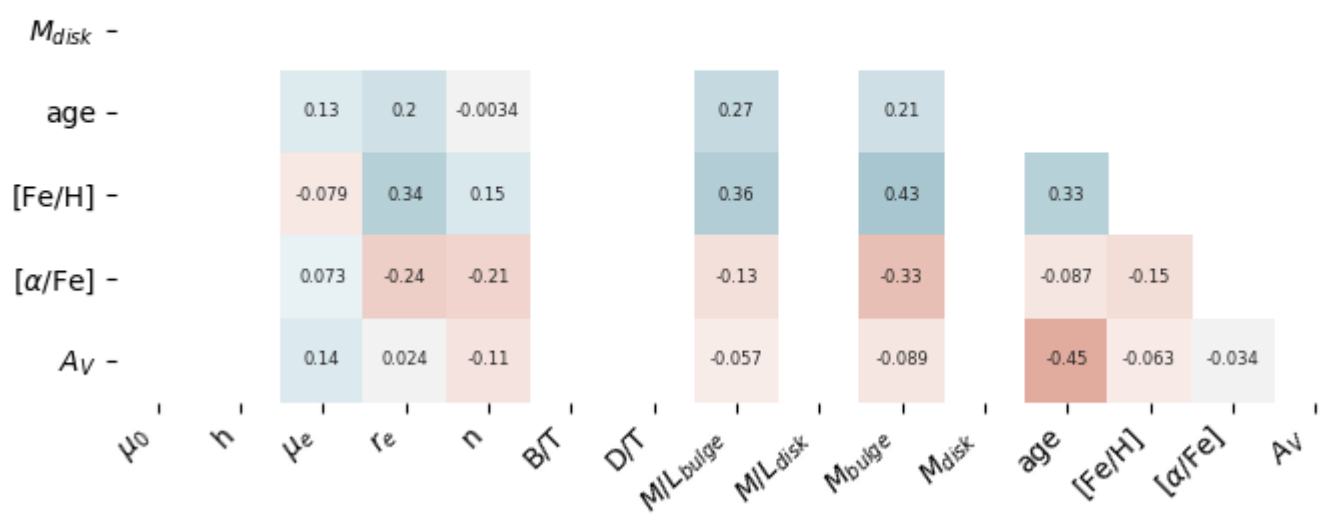

Figura D.13: Análogo à Figura 5.6, porém para galáxias elípticas sem AGN. 


\section{Galáxias barradas AGN}

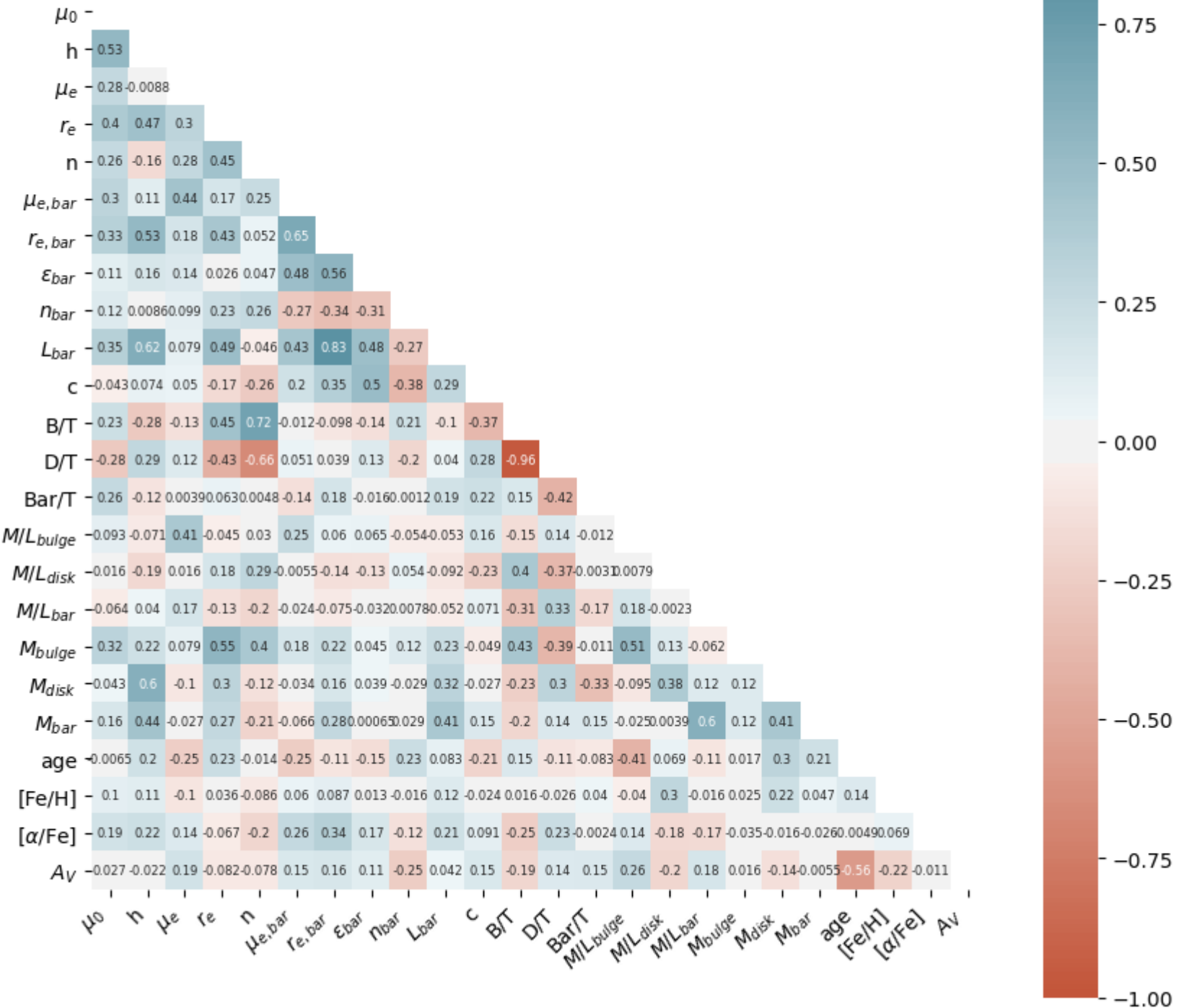

Figura D.14: Análogo à Figura 5.6 porém para galáxias espirais com barra com AGN. 


\section{Galáxias barradas sem AGN}

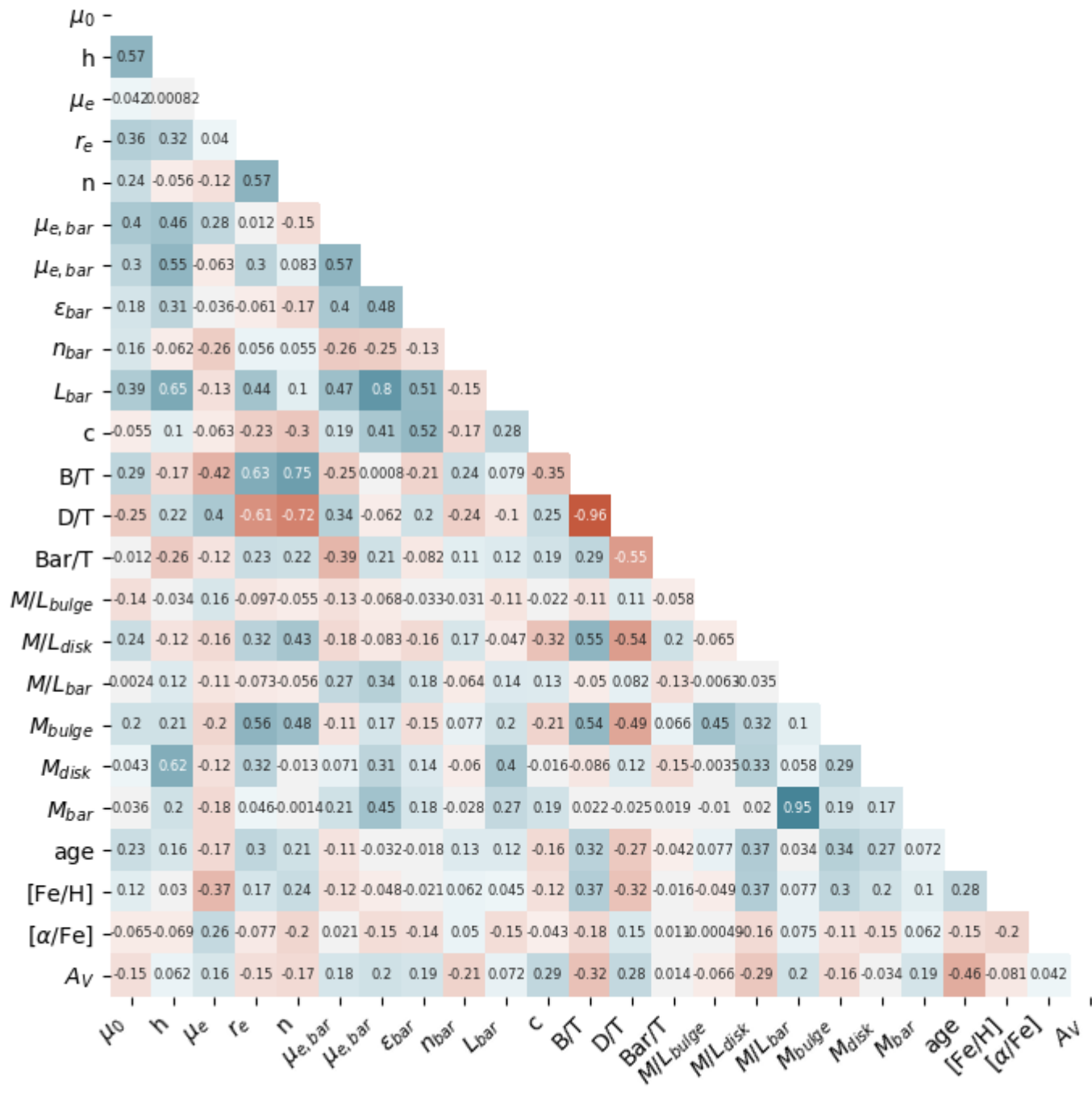

Figura D.15: Análogo à Figura 5.6, porém para galáxias espirais com barra sem AGN. 


\section{Galáxias sem barra AGN}

$\mu_{0}-$

$\mathrm{h}-0.47$

$\mu_{e}-0.3 \quad 0.014$

$r_{e}-0.28 \quad 0.29 \quad 0.31$

$\begin{array}{llll}\mathbf{n}-0.16 & -0.24 & 0.075 & 0.1\end{array}$

\begin{tabular}{l|l|l|l|l}
$\mathrm{B} / \mathrm{T}-0.19$ & -0.35 & -0.12 & 0.51 & 0.48
\end{tabular}

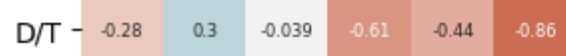

$\begin{array}{llllllll}M / L_{\text {bulge }}- & -0.1 & 0.0094 & 0.21 & -0.067 & -0.0063 & -0.19 & 0.21\end{array}$

$\begin{array}{lllllllll}M / L_{\text {disk }}-0.16 & 0.031 & 0.042 & 0.33 & -0.15 & 0.25 & -0.27 & -0.17\end{array}$

\begin{tabular}{|l|l|l|l|l|l|l|l|l}
\hline M bulge -0.067 & 0.29 & -0.076 & 0.58 & 0.15 & 0.32 & -0.3 & 0.51 & 0.11
\end{tabular}

\begin{tabular}{l|l|lllllllll} 
& $M_{\text {disk }}--0.048$ & 0.66 & -0.2 & 0.24 & -0.33 & 0.28 & 0.29 & 0.066 & 0.42 & 0.37
\end{tabular}

\begin{tabular}{lllll|l|l|l|l|l|l|l} 
& & & & & & & & \\
age -0.27 & 0.2 & -0.12 & 0.22 & 0.038 & 0.22 & -0.21 & -0.071 & 0.16 & 0.24 & 0.19
\end{tabular}

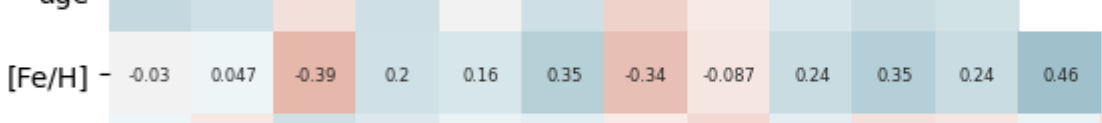

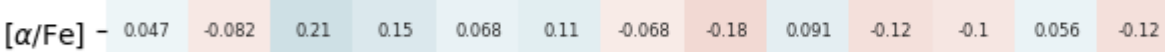

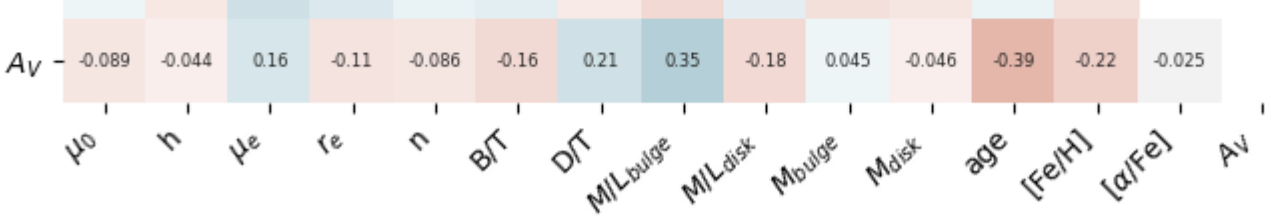

Figura D.16: Análogo à Figura 5.6 porém para galáxias espirais sem barra e com AGN. 


\section{Galáxias sem barra sem AGN}

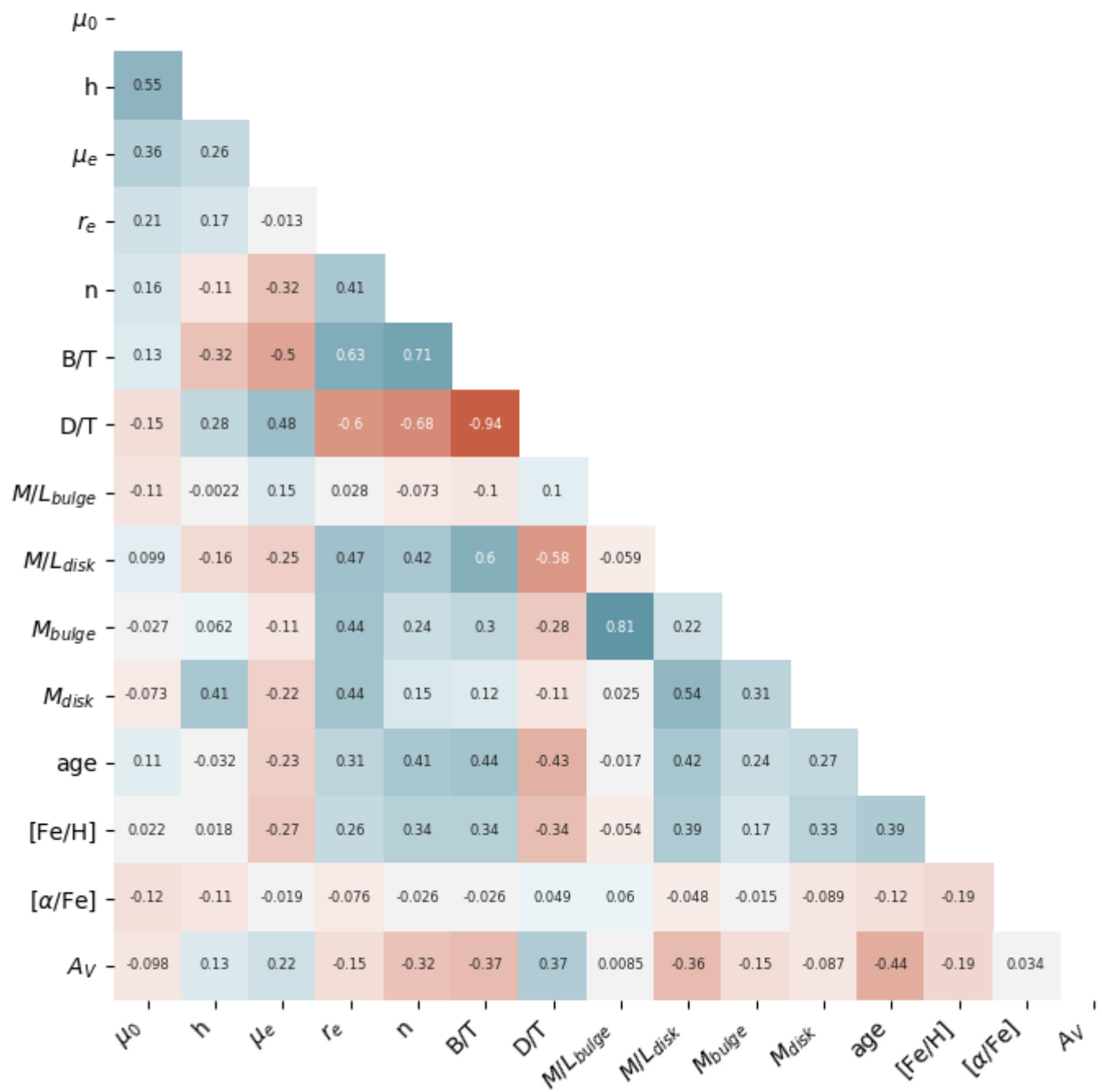

Figura D.17: Análogo à Figura 5.6 porém para galáxias espirais sem barra e sem AGN. 